Tuning the Surface Properties of Zeolites: Organosilane Coatings as Adsorption Modifiers

by

Irene Ana Maria Zuger, B. Sc.

\author{
A thesis submitted to \\ the Faculty of Graduate Studies and Research \\ in partial fulfillment of \\ the requirements for the degree of
}

\author{
Master of Science \\ Department of Chemistry \\ Carleton University \\ Ottawa, Ontario \\ August, 2002 \\ (c) Copyright \\ Irene Ana Maria Zuger, 2002
}


National Library of Canada

Acquisitions and Bibliographic Services

395 Wellington Street Ottawa ON K1A ON4 Canada
Bibliothèque nationale du Canada

Acquisitions et services bibliographiques

395, rue Wellington

Ottawa ON K1A ON4

Canada
The author has granted a nonexclusive licence allowing the National Library of Canada to reproduce, loan, distribute or sell copies of this thesis in microform, paper or electronic formats.

The author retains ownership of the copyright in this thesis. Neither the thesis nor substantial extracts from it may be printed or otherwise reproduced without the author's permission.
L'auteur a accordé une licence non exclusive permettant à la

Bibliothèque nationale du Canada de reproduire, prêter, distribuer ou vendre des copies de cette thèse sous la forme de microfiche/film, de reproduction sur papier ou sur format électronique.

L'auteur conserve la propriété du droit d'auteur qui protège cette thèse. $\mathrm{Ni}$ la thèse ni des extraits substantiels de celle-ci ne doivent être imprimés ou autrement reproduits sans son autorisation. 
The undersigned recommend to

the Faculty of Graduate Studies and Research

acceptance of the thesis

Tuning the Surface Properties of Zeolites:

Organosilane Coatings as Adsorption Modifiers

submitted by

Irene Ana Maria Zuger, B. Sc.

in partial fulfillment of the requirements for

the degree of

\section{Master of Science}

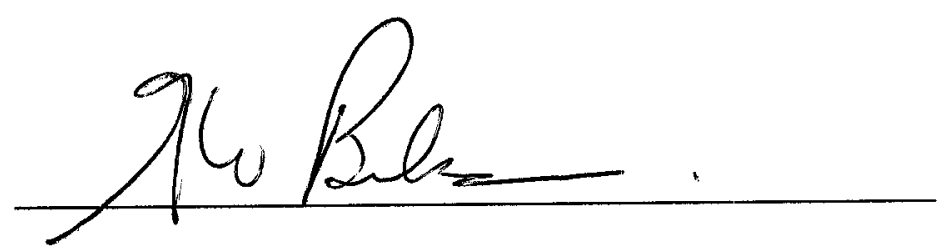

Chair, Department of Chemistry

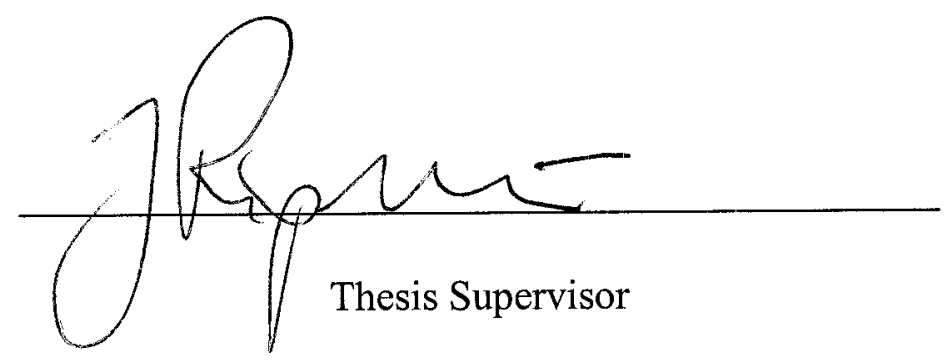

CARLETON UNIVERSITY

2002 


\begin{abstract}
Modification of the surface of zeolites with organosilane coating materials was performed through the introduction of a dried zeolite with an organosilanehydrocarbon solution resulting in a covalent linkage between coating and zeolite. Gravimetric techniques (TGA) and spectroscopic techniques (NMR) aided to identify the presence of coating material. This study looks specifically at modifying the surfaces and subsequent characterization of four zeolites: synthetic Linde Type A (LTA): $4 \AA$ and $5 \AA$, two natural zeolites that were obtained from sites in the Bay of Fundy, Nova Scotia, with the following coating materials: SurfaSil ${ }^{\mathrm{TM}}$ (SS), trimethylchlorosilane (TMCS) and a novel, synthetic pyrene-containing organosilane, pyrene-1-(4-butyl-dimethylchlorosilyl) ether (Pyrene-Cl). Through solvent uptake experiments with water, methanol and ethanol, it was found that the coated materials experienced the same degree of solvent uptake as the uncoated material. This was verified through preliminary TGA studies as well as duplicate uptake experiments. Solid state ${ }^{13} \mathrm{C}$ NMR experiments were done to verify the presence of the coating material by looking at the resonance of methyl carbons, specifically adjacent to silicon, shown at $0 \mathrm{ppm} .{ }^{29} \mathrm{Si}$ SS NMR showed that 5 chemical environments were found in both natural zeolite samples, indicating the presence of impurities. Powder X-ray diffraction also provided evidence of impurities within the samples; in addition, correlation of the sample powder patterns to literature values was not successful. ${ }^{27} \mathrm{Al}$ SS NMR showed that only tetrahedrally coordinated aluminum was present in both natural samples.
\end{abstract}




\section{Acknowledgments}

The completion of this thesis project would not have been possible were it not for the kind and generous support of many people. To begin, my deepest thanks are extended to my supervisor, Dr. John A. Ripmeester, for allowing me the opportunity to complete my work at the National Research Council of Canada, and for having the patience to guide me through the work from start to finish. I owe Dr. Stephen Lang my heartfelt gratitude for allowing me to run my own solid-state NMR experiments, and for being there to get through the sticky points. Thanks are extended to Don Leek for running my solution NMR samples; the spectra always turned out beautifully. Thanks are extended to Catherine Reinhold for helping me try to make sense of my powder patterns. Many thanks to my editors and brainstorm group members: Philip Brown, Dalbinder Colman, Dr. Pierre Desjardins, Dr. Stephen Lang, Dr. Dimitry Soldatov, Dr. Viktor Terskikh and Dr. Lee Wilson. Your eyes saw more than mine ever could, and your ideas proved most valuable. To the members of Functional Materials, I thank you for always making the National Research Council a wonderful place to work; the past four years have proven to be an invaluable experience for me. I will take the memories with me throughout my travels, and hope to always have a wonderful team of coworkers wherever my career shall take me. 


\section{Dedication}

\section{OThis thesis is dedicated to}

myparents, Davas and Seargie, my brothex Christopher,

$$
\text { and my love, Pavax } \mathscr{B} \text { aldex. }
$$

Without you, Of would never have made it this fax.

OHata na sue! 


\section{Table of Contents}

Title page

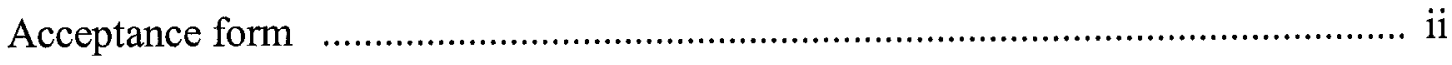

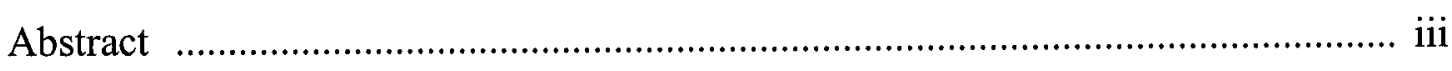

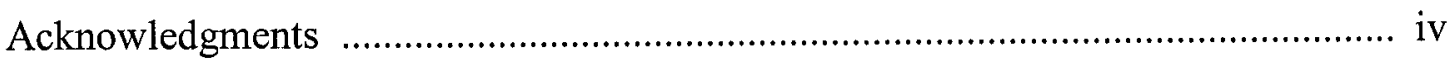

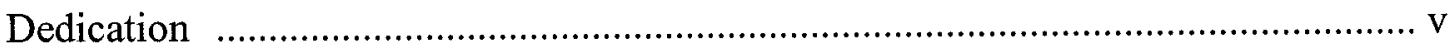

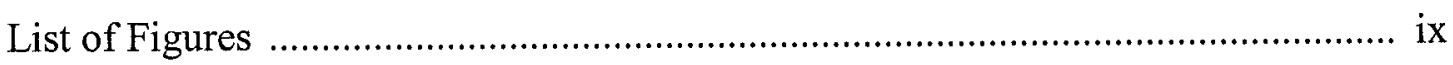

List of Tables

List of Abbreviations

\section{Chapter I: General Introduction to Zeolites: Formation, Modification} and Implications ……………………………………........... I-1

1. Introduction: a brief history ..................................................... I-2

1.1 The structure of a zeolite ............................................................ I-3

1.1.1 Basic building blocks ...................................................... I-4

1.1.2 Zeolite groups ......................................................... I-4

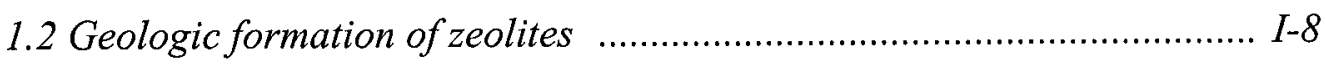

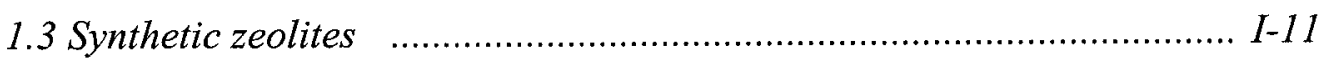

1.3.1 Laboratory preparation ............................................... I-12

1.3.2 Commercial preparation ............................................... I-14

1.4 Industrial application of zeolites ............................................. I-16

1.4.1 Catalysis ...................................................................... I-19

1.4.2 Adsorption ............................................................... I-21

1.4.3 Ion exchange ............................................................... I-24

1.5 Zeolites in toxicology .............................................................. I-25

1.6 Zeolites studied $\quad$.................................................................... I-26

1.6.1 Linde Type A (LTA) ................................................. I-26

1.6.2 Mordenite $(M O R)$.................................................... I-28 


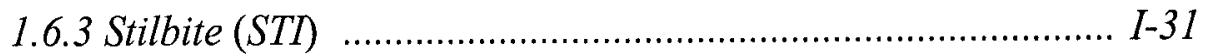

1.7 Limitations of current zeolite knowledge ........................................... I-31

1.8 Thesis overview ………………………….............................. I-33

References

Chapter II: Experimental Techniques …………............................... II-1

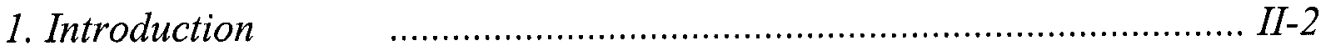

1.1 Thermal Gravimetric Analysis (TGA) ............................................. II-2

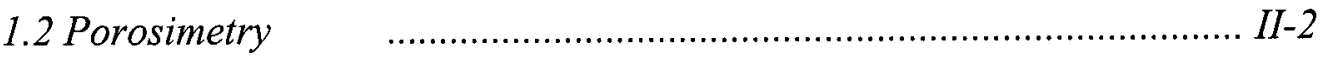

1.3 Powder X-ray Diffraction (PXRD) ………………….................... II-3

1.4 Solid-state Nuclear Magnetic Resonance Spectroscopy (SS NMR) ....... II-4

1.4.1 ${ }^{29} \mathrm{Si}$ NMR ...................................................................... II-6

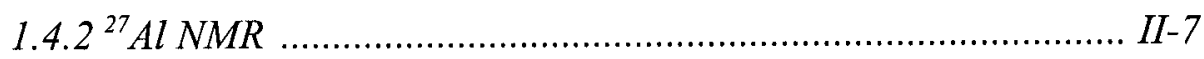

2. Experimental methods ………………........................................... II-10

2.1 TGA experiments .................................................................. II-10

2.1.1 Sample weight loss measurements …………………......... II-10

2.1.2 Temperature cycle experiments ........................................ II-10

2.2 Porosimetry measurements .......................................................... II-11

2.3 PXRD measurements ................................................................. II-11

2.4 SS MAS NMR spectroscopy methods ............................................... II-11

2.4.1 ${ }^{13} \mathrm{C}$ SS MAS NMR experiments .......................................... II-11

2.4.2 ${ }^{29} \mathrm{Si}$ SS MAS NMR experiments ........................................... II-11

2.4. ${ }^{27} \mathrm{Al}$ SS MAS NMR experiments ………………………...... II-12

2.5 General procedure for the coating of zeolites ………………........... II-12

2.5.1 Pre-coating of glass vessels .............................................. II-13

2.6 General Pyrene-derivative Synthetic Procedure …………………... II-13

2.6.1 Synthesis of Pyrene-TBDMS ………………………......... II-14

2.6.2 Synthesis of Red-Pyrene-TBDMS ...................................... II-14

2.6.3 Synthesis of Pyrene-But-OH ………………………….... II-15

2.6.4 Synthesis of Pyrene- $\mathrm{Cl}$.................................................... II-15

2.7 Adsorption experiment setup ..................................................... II-16 
References

II-17

Chapter III: Modification of surfaces and the synthesis of a novel coating material …………………................................... III-1

1. Modification of surfaces …………….......................................... III-2

1.1 Silane coating chemistry ................................................... III-3

1.2 Coating of glass surfaces ............................................... III-5

1.3 Modification of zeolites and zeolite-type materials ............... III-6

2. Synthesis of a new coating material: Pyrene-Cl .............................. III-6

2.1 Synthetic strategy .......................................................... III -7

3. Synthesis of a novel pyrene coating: results .................................. III-11

References $\quad$............................................................... III 15

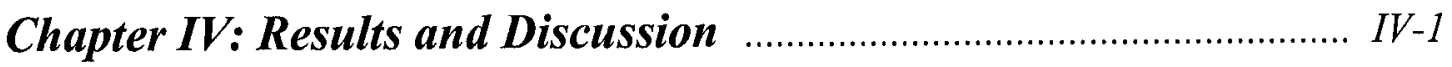

1. Thermal Gravimetric Analysis (TGA) ........................................ IV-2

1.1 Initial survey of weight loss of coated vs non-coated zeolites ............................................................... IV-2

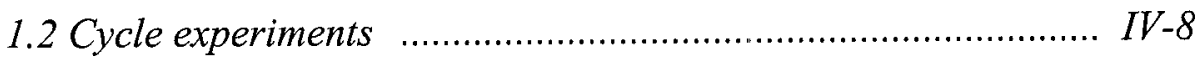

2. Porosimetry $\quad$................................................................ IV 15

3. Powder X-Ray diffraction (PXRD) of the natural zeolite samples $\ldots . . . I V-17$

4. Solid State Nuclear Magnetic Resonance (NMR) Spectroscopy ......... IV-21

$4.1{ }^{13}$ C SS NMR spectroscopy of coated LTA $4 \AA \ldots \ldots \ldots \ldots \ldots \ldots \ldots . . . I V-21$

$4.2{ }^{29} \mathrm{Si}$ SS NMR spectroscopy of the natural zeolite samples ...... IV-21

$4.3{ }^{27} \mathrm{Al} \mathrm{SS} \mathrm{NMR} \mathrm{spectroscopy} \mathrm{of} \mathrm{the} \mathrm{natural} \mathrm{zeolite} \mathrm{samples.} \mathrm{....} \mathrm{IV-28}$

5. Uptake experiments ........................................................... IV-28

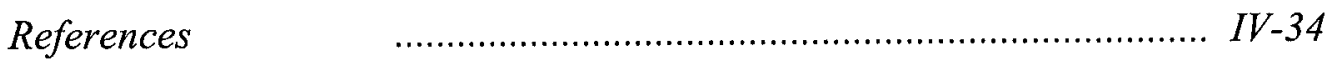

Chapter V: Conclusions .................................................................... $V-1$

1. Concluding remarks .................................................................

2. Future work $\quad$.................................................................... $V-4$

Appendix: Additional Data ............................................................. $A-1$ 


\section{List of Figures}

\begin{tabular}{|c|c|}
\hline Figure & Caption \\
\hline Fig. 1.1 & $\begin{array}{l}\text { Basic structural units of zeolites: a, tetrahedron with silicon } \\
\text { atom at the centre and oxygen atoms at the apexes; b, } \\
\text { tetrahedron with aluminum atom and attached monovalent } \\
\text { cation compensating for the charge imbalance between the } \\
\text { two metal centres in a multiple tetrahedron chain. }\end{array}$ \\
\hline
\end{tabular}

Fig. 1.2 Secondary building units (SBUs) in zeolite structures. The black dots represent the central metal atom in the tetrahedra.

Fig. 1.3 Polyhedra of zeolite frameworks: a, the $\beta$ cage, or truncated octahedron; $\mathrm{b}$, the $\alpha$ cage, or truncated cuboctahedron.

Fig. 1.4 A simple scheme depicting hydrothermal zeolite synthesis from a reactive aluminosilicate gel at temperatures above $90^{\circ} \mathrm{C}$.

Fig. 1.5 A hydrothermal bomb in a configuration with removable internal attachments and a heating element for preparing giant zeolite crystals through a temperature gradient and nutrient.

Fig. 1.6 A schematic representation of the preparation and crystallization of a $\mathrm{Na}_{2} \mathrm{O} \cdot \mathrm{Al}_{2} \mathrm{O}_{3} \cdot \mathrm{SiO}_{2} \cdot \mathrm{H}_{2} \mathrm{O}$ system gel.

Fig. 1.7 The reactions involved in the clay conversion process starting with kaolin to produce zeolite A.

Fig. 1.8 Generation of Brønsted and Lewis acid sites in zeolites.

Fig. 1.9 Framework of LTA viewed along [100]. Blue spheres indicate cation positions within the framework structure.

Fig. 1.10 Framework of MOR viewed along [001]. I-30

Fig. 1.11 Framework of STI viewed along [100]. I-32

Fig. 2.1 Ranges of ${ }^{29} \mathrm{Si}$ chemical shifts of $\mathrm{Q}^{4}(n \mathrm{Al})$ units in II-9 aluminosilicates.

Fig. 3.1 A schematic representation of the reaction of a coating III-4 material with a substrate. 
Fig. 3.2 Ball-and-stick model, with van der Waal's radii, III-8 representation of the organosilane coating materials: a, trimethylchlorosilane; b, SurfaSil ${ }^{\mathrm{TM}} ; \mathrm{c}$, pyrene-1-(4-butyldimethylchlorosilyl) ether.

Fig. 3.3 Sequence of steps for the synthesis of pyrene-1-(4-butylIII-9 dimethylchlorosilyl) ether; the last step shows the introduction of the material to a zeolite surface.

Fig. 3.4 ${ }^{1} \mathrm{H}$ NMR spectrum of Pyrene-Cl (5). The annotation is defined as: ' $\mathrm{p}$ '= product.

Fig. 3.5 Mass spectrum of Pyrene-Cl (5).

III-14

Fig. 4.1 Ball and stick models of : a, water ( $1.5 \AA$ A length; $b$, methanol ( $\sim 3.5 \AA$ length); $c$, ethanol ( $\sim 4.5 \AA$ length). Molecules shown are to illustrate how slight difference in size can alter diffusion into a zeolite.

Fig. 4.2 Space filling models of water, methanol and ethanol molecules. The top diagram illustrates the difference in length; from left to right: water, methanol, ethanol. The bottom diagram shows a side view, where the cross-sectional diameter of all three molecules is similar.

Fig. 4.3 Cycle experiment for zeolite LTA 4Å. The green curve is the neat sample, the blue is SS-coated sample, and the red is the TMCS-coated sample. Where the curves show a decrease in mass, the temperature is being increased to $350^{\circ} \mathrm{C}$; conversely, where the curve shows an increase, the temperature is being decreased to $25^{\circ} \mathrm{C}$.

Fig. 4.4 Cartoon representation of the possible behaviour of the coating material, and the suspected ability to trap water or other solvent molecules (small blue spheres).

Fig. 4.5 TGA plot of a cycle experiment with LTA $4 \AA$ (blue curve) and $5 \AA$ (red curve) with helium as the flow gas. Weight loss denotes temperature increase; conversely, weight gain denotes temperature decrease.

Fig. 4.6 PXRD pattern of NS1; JCPDS peaks for MOR are shown in red.

Fig. 4.7 PXRD pattern of NS2; JCPDS peaks for STI are shown in IV-20 red.

Fig. $4.8 \quad{ }^{13} \mathrm{C}$ SS NMR spectrum of SS-coated zeolite LTA 4A. 
Fig. 4.9 ${ }^{29}$ Si SS NMR spectrum of NS1. Asterisks denote the peaks that closely match the literature values.

Fig. $4.10{ }^{29} \mathrm{Si}$ SS NMR spectrum of NS2. Asterisks denote the peaks IV-24 that closely match the literature values.

Fig. $4.11{ }^{27} \mathrm{~A} 1$ SS NMR spectra of NS1 (top) and NS2 (bottom). IV-27 Spinning sidebands are shown with asterisks.

Fig. 4.12 Uptake experiments for LTA $4 \AA$ and LTA $5 \AA$ exposed to IV-29 water at $97 \%$ humidity.

Fig. 4.13 Uptake experiments for LTA $4 \AA$ and LTA $5 \AA$ exposed to IV-30 methanol.

Fig. 4.14 Uptake experiments for LTA $4 \AA$ and LTA $5 \AA$ exposed to IV-31 ethanol.

Fig. A.1 ${ }^{1} \mathrm{H}$ NMR spectrum of PBA (1). A-2

Fig. A.2 $\quad{ }^{1}$ H NMR spectrum of Pyrene-TBDMS (2). A-3

Fig. A.3 $\quad{ }^{1}$ H NMR spectrum of Red-Pyrene-TBDMS (3). A-4

Fig. A.4 $\quad{ }^{1}$ H NMR spectrum of Pyrene-But-OH (4). A-5

Fig. A.5 Weight loss experiment TGA plot for LTA $4 \AA$ exposed to A-6 water vapour.

Fig. A.6 Weight loss experiment TGA plot for LTA $4 \AA$ exposed to A-13 methanol.

Fig. A.7 Weight loss experiment TGA plot for LTA $4 \AA$ exposed to A-14 ethanol.

Fig. A.8 Temperature cycle experiment TGA plot for LTA $5 \AA$ A-15

Fig. A.9 Temperature cycle experiment TGA plot for LTA $4 \AA$ and $5 \AA, \quad$ A-16 coated with Pyrene-Cl.

Fig. A.10 Temperature cycle experiment TGA plot for NS1. A-17

Fig. A.11 Temperature cycle experiment TGA plot for NS2. A-18

Fig. A.12 Weight loss experiment TGA plot for $4 \AA$, where the final A-19 temperature was $1000^{\circ} \mathrm{C}$, under oxygen flow.

Fig. A.13 Weight loss experiment TGA plot for $5 \AA$, where the final A-20 temperature was $1000^{\circ} \mathrm{C}$, under oxygen flow.

Fig. A.14 Weight loss experiment TGA plot for NS1, where the final A-21 temperature was $1000^{\circ} \mathrm{C}$, under oxygen flow. 
Fig. A.15 Weight loss experiment TGA plot for NS2, where the final temperature was $1000^{\circ} \mathrm{C}$, under oxygen flow. 


\section{List of Tables}

\section{Table}

Caption

Page

Table 1.1 A listing of the typical classification of the different zeolite groups based on the main component of the zeolite structure.

Table 1.2 Some dimensional properties of common zeolites, where window size dictates whether molecules may be taken up by the zeolite structure.

Table 4.1 Total weight loss as a \% where the final temperature was $1000{ }^{\circ} \mathrm{C}$. Values are based on original weight.

Table 4.2 Total weight loss of zeolite samples after exposure to solvent atmosphere. Values are based on original weight. For the NS1 and NS2 values, the reported literature value is given in parentheses.

Table 4.3 Initial water loss and initial gain of gas during the first cycle of temperature cycle experiments, where the maximum temperature was $350^{\circ} \mathrm{C}$, and the minimum $25^{\circ} \mathrm{C}$. Values are based on original weight.

Table 4.4 Average water loss and average gain of gas during IV-11 temperature cycle experiments, where the maximum temperature was $350{ }^{\circ} \mathrm{C}$, and the minimum $25^{\circ} \mathrm{C}$. Values are based on original weight.

Table 4.5 Porosimeter data for NS1 and NS2, showing calculated surface area, pore diameter and pore volume.

Table 4.6 ${ }^{29} \mathrm{Si}$ experimental chemical shifts of NS1 and NS2 in comparison to the literature values. Chemical shifts are correct to within $10 \%$ error.

Table 4.7 Ratio of moles of solvent per mole of zeolite LTA.

Table A.1 PXRD data for MOR. 


\section{List of Abbreviations}

SBU

S4R

S6R

S8R

S10R

S12R

D4R

D6R

LTA

MOR

STI

SS

TMCS

TBDMS

TBAF

DCDMS

Neat

PBA

Pyrene-TBDMS

Red-Pyrene-TBDMS

Pyrene-But-OH

Pyrene-Cl

TGA

PXRD

NMR
Secondary Building Unit

Single 4-ring

Single 6-ring

Single 8-ring

Single 10-ring

Single 12-ring

Double 4-ring

Double 6-ring

Linde Type A zeolite

Mordenite

Stilbite

SurfaSil ${ }^{\mathrm{TM}}$

trimethylchlorosilane

t-butyldimethylsilyl chloride

tetrabutylammonium fluoride

dichlorodimethylchlorosilane

non-coated zeolite

Pyrene-1-butyric acid

Pyrene-1-(4-butyl-t-butyldimethylsilyl)ester

Pyrene-1-(4-butyril-t-butyldimethylsilyl)ether

Pyrene-1-(4-butanol)

Pyrene-1-(4-butyl-dimethylchlorosilyl)ether

Thermal Gravimetric Analysis

Powder X-ray Diffraction

Nuclear Magnetic Resonance 
SS

$\mathrm{CP}$

MAS

JCPDS

NS1

NS2
Solid-state

Cross-polarization

Magic angle spinning

Joint Committee on Powder Diffraction Standards

Natural sample 1, thought to be Mordenite

Natural sample 2, thought to be Stilbite 


\section{Chapter I}

General Introduction to Zeolites: Formation, Modification and Implications

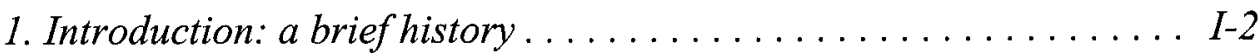

1.1 The structure of a zeolite $\ldots \ldots \ldots \ldots \ldots \ldots \ldots \ldots \ldots \ldots$

1.1.1 Basic building blocks ............... I-4

1.1.2 Zeolite groups $\ldots \ldots \ldots \ldots \ldots \ldots \ldots \ldots \ldots \ldots \ldots$

1.2 Geologic formation of zeolites $\ldots \ldots \ldots \ldots \ldots \ldots \ldots \ldots$

1.3 Synthetic zeolites $\ldots \ldots \ldots \ldots \ldots \ldots \ldots \ldots \ldots \ldots \ldots \ldots \ldots$

1.3.1 Laboratory preparation $\ldots \ldots \ldots \ldots \ldots \ldots \ldots \ldots \ldots$

1.3.2 Commercial preparation .............. I-14

1.4 Industrial application of zeolites $\ldots \ldots \ldots \ldots \ldots \ldots \ldots \ldots$

1.4 .1 Catalysis . . . . . . . . . . . . . . . . I-19

1.4 .2 Adsorption $\ldots \ldots \ldots \ldots \ldots \ldots \ldots \ldots \ldots \ldots \ldots \ldots$

1.4 .3 Ion exchange $\ldots \ldots \ldots \ldots \ldots \ldots \ldots \ldots \ldots \ldots \ldots \ldots$

1.5 Zeolites in toxicology $\ldots \ldots \ldots \ldots \ldots \ldots \ldots \ldots \ldots \ldots \ldots \ldots$

1.6 Zeolites studied $\ldots \ldots \ldots \ldots \ldots \ldots \ldots \ldots \ldots \ldots \ldots \ldots \ldots$

1.6.1 Linde Type A (LTA) . . . . . . . . . . . . . I-26

1.6.2 Mordenite $(M O R) \ldots \ldots \ldots \ldots \ldots \ldots \ldots \ldots \ldots \ldots$

1.6.3 Stilbite $(S T I) \ldots \ldots \ldots \ldots \ldots \ldots \ldots \ldots \ldots \ldots \ldots$

1.7 Limitations of current zeolite knowledge $\ldots \ldots \ldots \ldots \ldots$ I-31

1.8 Thesis overview $\ldots \ldots \ldots \ldots \ldots \ldots \ldots \ldots \ldots \ldots \ldots \ldots \ldots$

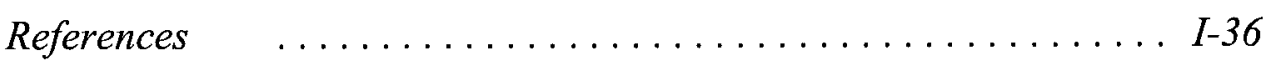




\section{Introduction : a brief history}

Since the discovery of stilbite in 1756 by Freiherr Axel Fredrik Cronstedt of Sweden, zeolites have become an area of interest for earth scientists, mineralogists and chemists, in discovery, chemical and industrial uses. The word zeolite is derived from the Greek words $\operatorname{zein}(\zeta \varepsilon \imath v)$, which means "to boil" and lithos $(\lambda \iota \theta 0 \varsigma)$, "stone", in allusion to their frothing characteristics when heated. They are very common and well recognized as fine crystals of hydrothermal genesis in geodes and fissures of eruptive rocks, or as microcrystalline masses of sedimentary origin. ${ }^{1}$

Zeolites are aluminosilicates with the general formula $\left(\mathrm{M}^{\mathrm{n}+}\right)_{\mathrm{x} / \mathrm{n}}\left[\left(\mathrm{AlO}_{2}\right)_{\mathrm{x}}\left(\mathrm{SiO}_{2}\right)_{\mathrm{y}}\right] \cdot \mathrm{zH}_{2} \mathrm{O}$, where $\mathrm{n}$ is the charge of the metal cation, $\mathrm{M}^{\mathrm{n}+}$, which is usually $\mathrm{Na}^{+}, \mathrm{K}^{+}$or $\mathrm{Ca}^{2+}, \mathrm{x}$ and $\mathrm{y}$ are the total number of tetrahedra per unit cell and $z$ is the number of moles of water of hydration, which can vary. Zeolties possess a framework structure with enclosing cavities occupied by large ions and water molecules, and allow for considerable movement and freedom of included guest species, permitting ion exchange and reversible dehydration, without structural degradation. ${ }^{2}$

After Cronstedt's initial finding, other researchers followed the path of this discovery. In $1845, \mathrm{Way}^{3}$ ascertained that certain soils retained ammonium salts. Breck ${ }^{4}$ reported that hydrated silicates in the soil were found to be responsible for this phenomenon and these were probably the first ion-exchange experiments. Weigel and Steinhoff ${ }^{5}$, in 1925 , were the first to determine that chabazite selectively absorbed smaller organic molecules and rejected 


\section{Chapter I General Introduction to Zeolites: Formation, Modification and Implication}

large molecules. This characteristic behaviour was described by McBain ${ }^{6}$ in 1932 as 'molecular sieving'. As knowledge of the properties of zeolites gradually increased, it became apparent that they could be utilized for industrial processes; nevertheless, zeolites were considered to be mineralogical curiosities that filled small cavities called vugs, and fractures in igneous rocks. ${ }^{7}$ It was not until the 1940s and 1950s that research on the properties of zeolites increased dramatically. ${ }^{7}$ Large tonnages of natural zeolites were not discovered until the late 1950 s when various researchers reported on vast sedimentary deposits in the Western United States. Prior to this time, emphasis was placed on the synthesis of zeolites for commercial use. ${ }^{8}$ There are many natural zeolites, some of which have been synthesized. Of those that have been synthesized, many zeolite structures do not occur in nature.

Zeolites are undoubtedly one of the most important and interesting framework silicates due to the inherent characteristic porous $\left[(\mathrm{Al}, \mathrm{Si}) \mathrm{O}_{2}\right]_{\mathrm{n}}$ framework. The porosity of zeolites enables them to perform several functions, namely in ion exchange and selective adsorption applications.

\subsection{The structure of a zeolite}

As previously mentioned, zeolites are a well-defined class of crystalline, naturally occurring aluminosilicate minerals. They have infinite, three-dimensional structures arising from a framework of $\mathrm{SiO}_{4}$ and $\mathrm{AlO}_{4}$ coordination polyhedra linked through their vertices. The frameworks generally are open and contain channels and cavities in which cations and 
water molecules are located. ${ }^{9}$ These channels and cavities form three-dimensional frameworks that have the ability to house guests molecules within their interiors.

\subsubsection{Basic building blocks}

The basic units of a zeolite are the $\mathrm{SiO}_{4}$ or $\mathrm{AlO}_{4}$ tetrahedra, where the $\mathrm{Si}$ or $\mathrm{Al}$ atom resides in the centre, and the oxygen atoms form the vertices (Fig. 1.1). If an aluminum atom is present rather than a silicon atom, a positive metal ion is required to maintain a charge balance. These tetrahedra are then assembled together to form secondary building units (SBUs). There are eight types of SBU: the single 4-ring (S4R), the single 6-ring (S6R), the single 8-ring (S8R), the double 4-ring (D4R), the double 6-ring (D6R), the natrolite unit (41), the mordenite unit (5-1) and the stilbite unit (4-4-1) (Fig. 1.2). When depicted as ball and stick models, only the central atoms are shown for clarity. The D4R and D6R units are obvious combinations of the S4R and S6R units. In some cases, the zeolite framework can be considered in terms of polyhedral units, such as the truncated octahedron (Fig. $1.3 \mathrm{a}$ ); these are cage-like units designated by the Greek letters: $\alpha, \beta, \gamma$, etc. ${ }^{10}$ The $\alpha$-cage refers to the largest unit - the truncated cuboctahedron (Fig. $1.3 \mathrm{~b}$ ), and so on. The SBUs can then subsequently be assembled to form a variety of polyhedra, forming the 133 different zeolite structures that are known today. ${ }^{11}$

\subsubsection{Zeolite Groups}

Zeolite classification has evolved with increasing knowledge about their structures, while earlier classification systems were based on morphology and had zeolite species 

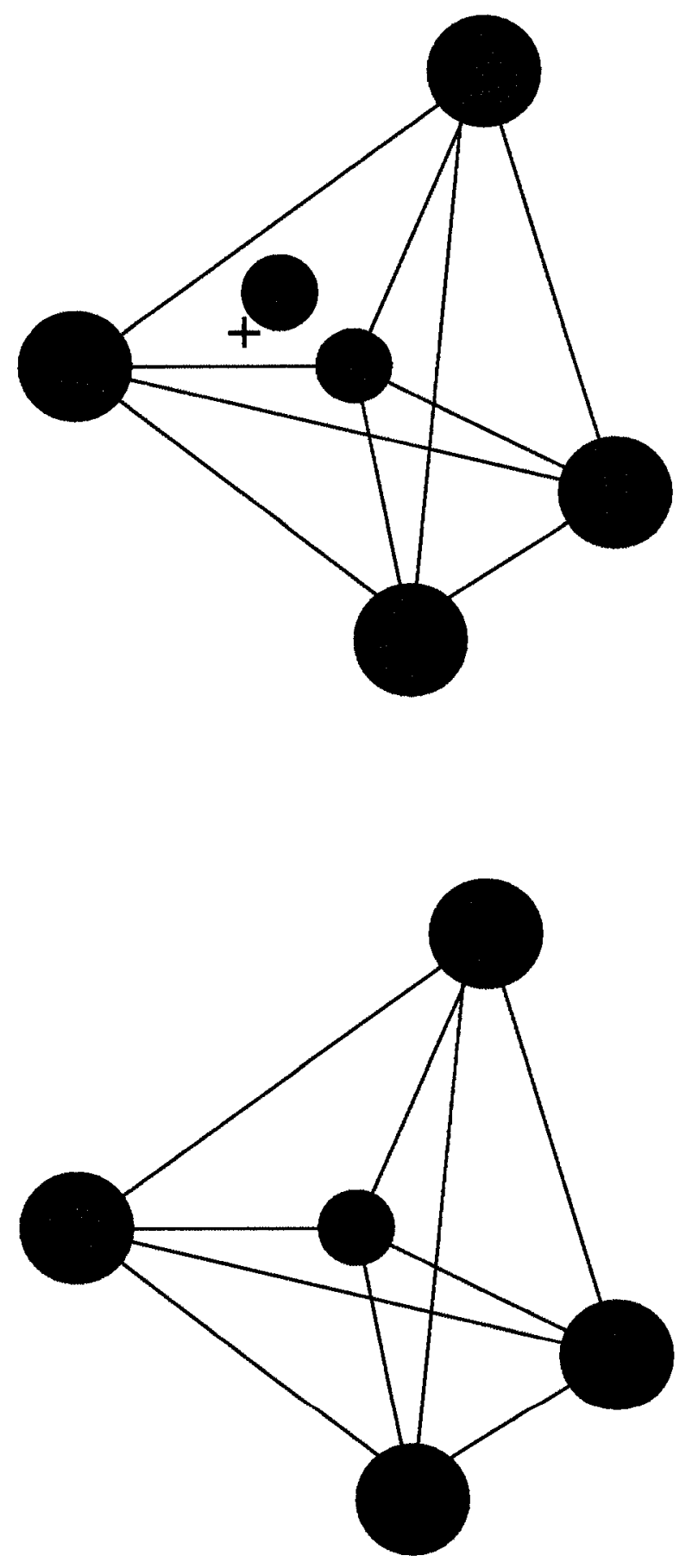

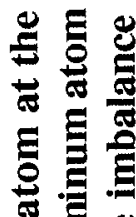

0

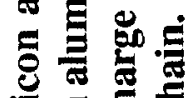

$\because=\frac{5}{0}$

동

$<$ 용

응

해요용

을

용를

๙ 8

过

冚恶

娄

동

3 훙

클을

해용

Eั

응

6

흔

บ

䍃

$\underset{7}{ت}$ 


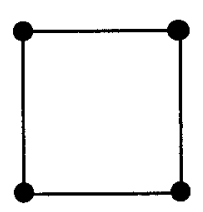

S4R

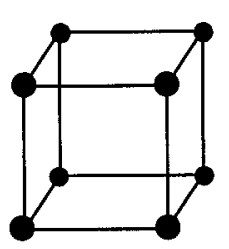

D4R

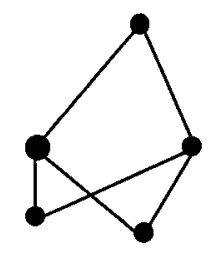

$\mathrm{T}_{5} \mathrm{O}_{10} 4-1$

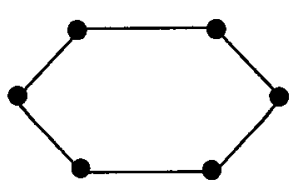

S6R

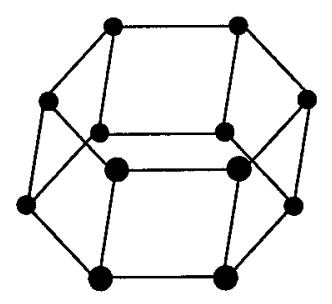

D6R

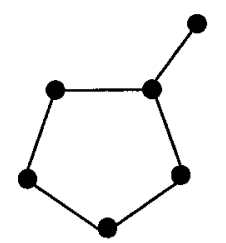

$\mathrm{T}_{8} \mathrm{O}_{16} 5-1$

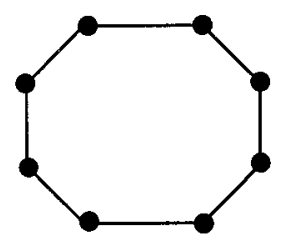

S8R

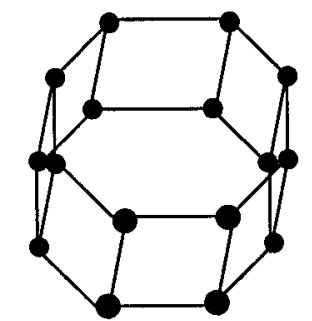

D8R

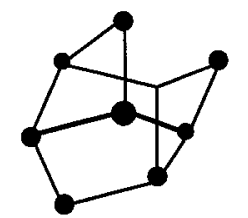

$\mathrm{T}_{10} \mathrm{O}_{20} 4-4-1$

Fig 1.2 Secondary building units (SBUs) in zeolite structures. The black dots represent the central metal atom in the tetrahedra. 


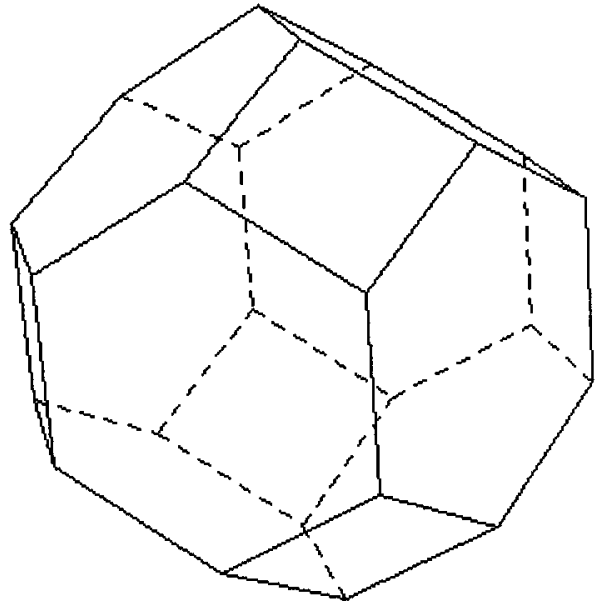

(a)

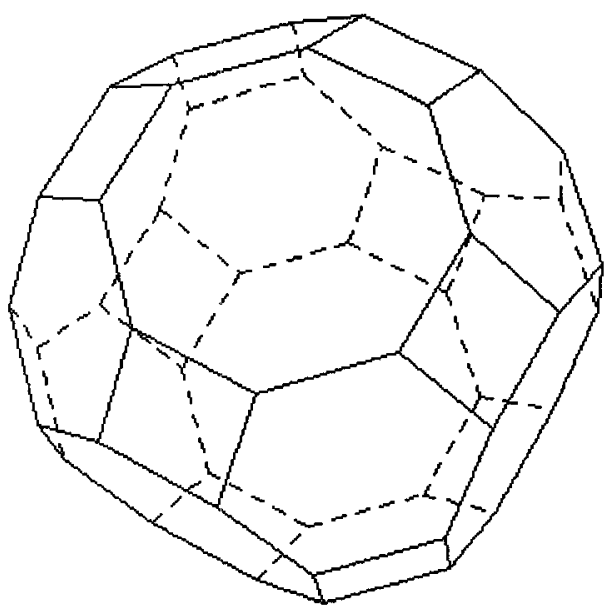

(b)

Fig 1.3 Polyhedra of zeolite frameworks: a, the $\boldsymbol{\beta}$ cage, or truncated octahedron; $b$, the $\alpha$ cage, or truncated cuboctahedron. 
grouped together through commonalities such as texture, colour, and crystal habit. Current classifications of zeolites are based on the framework topology. Breck ${ }^{12}$ grouped zeolites by common SBUs into seven categories (Table 1.1). It should be noted that not all zeolites are typical within a group because of slight differences in chemistry and crystallography. Hence, the completed framework structures of the zeolites are composed of the permutations of assemblies of tetrahedra in building units which range from the simple 4-rings to large polyhedra.

\subsection{Geologic formation of zeolites}

Zeolties occur in rocks of many types and in a wide variety of geologic settings. Most of the earlier geological work on zeolites was carried out on typical occurrences in cavities of basalt flows. In the 1950s, however, detailed X-ray diffraction studies of bedded tuffs in the Western United States, Japan and Italy showed large quantities of zeolites in these sedimentary rocks. ${ }^{13}$ According to $\mathrm{Hay}^{14}$, most naturally occurring zeolites are assignable to six types of geologic environments or hydrological systems: (1) saline/alkaline lakes, (2) saline, alkaline soils and land surfaces, (3) sea-floor sediments, (4) percolating water in an open hydrologic system, (5) hydrothermal alteration and (6) burial diagenesis. Two additional geological occurrences of natural zeolite formation have been added to the list by Iijima ${ }^{15}$; magmatic zeolites and zeolites formed in impact craters.

The first, saline/alkaline lakes, where block-faulted terrains and trough valleys associated with continental rifting establish a closed basin system and control the transport 
Table 1.1 A listing of the typical classification of the different zeolite groups based on the main component of the zeolite structure.

\begin{tabular}{cl} 
Group & Secondary Building Unit (SBU) \\
\hline 1 & Single 4-ring, S4R \\
2 & Single 6-ring, S6R \\
3 & Double 4-ring, D4R \\
4 & Double 6-ring, D6R \\
5 & Complex 4-1, $\mathrm{T}_{5} \mathrm{O}_{10}$ unit \\
6 & Complex 5-1, $\mathrm{T}_{8} \mathrm{O}_{16}$ unit \\
7 & Complex 4-4-1, $\mathrm{T}_{10} \mathrm{O}_{20}$ unit
\end{tabular}




\section{Chapter I General Introduction to Zeolites: Formation, Modification and Implication}

of clastic material beyond the basin edges; the alkaline zeolites form in these restricted lakes. Alalcime, clinoptilolite and mordenite, among others, commonly form where $\mathrm{pH}$ values are around that of $9.5^{13}$

The next environment for the formation of zeolites can be found in saline/alkaline soils and land surfaces, where climate is the controlling factor in the formation of zeolites. These deposits form in arid and semi-arid regions where evaporation causes sodium carbonate-bicarbonate to concentrate in the top layers of the soil. Rainwater percolates through, dissolving the formed salts and thereby increasing the $\mathrm{pH}$, allowing it to alter silica glasses and aluminosilicates present in the soil. ${ }^{13}$ Some examples of zeolites that are found are analcime, the most common, followed by smaller amounts of phillipsite, natrolite and chabazite.

The third environment is found in sea-floor sediments, under low temperatures and moderate $\mathrm{pH}$ conditions, ranging from $\mathrm{pH}$ 7-8. Zeolites in the slow sedimentation regions of the Pacific and Indian Oceans occur in post-Miocene (23-5 million years ago, mya) brown clays, vitric siliceous and calcareous oozes and basaltic volcanic sediments. Here phillipsite is the dominant species. In the Atlantic and Pacific margin can be found calcareous sediments and terrigenous clays of Paleogene (65-24 mya) and Cretaceous ages (144-65 mya), where clinoptilolite is the dominant species. Zeolites in sea-floor sediments form by the reaction of glasses with pore water. ${ }^{13}$

The fourth geologic instance describes the process of percolating water in an open 
hydrologic system through porous pyroclastic materials rich in reactive glass. Here the $\mathrm{pH}$ and dissolved solid content of the ground water increase as it reacts with the vitric ash, until zeolites are precipitated. Movement of the ground water downward through the system results in a vertical zonation of water composition and minerals, including zeolites. ${ }^{14}$ Typical striation that is found: an upper zone of fresh glass, montmorillonite and opal. The next bed can contain up to $90 \%$ clinoptilolite and the underlying zone can contain analcime, feldspar and quartz.

Another system is that of hydrothermal alteration, where zeolites precipitate from alkaline to weakly acidic hot water. ${ }^{15}$ The assemblies observed are controlled by temperature, host rock composition and permeability, and geothermal fluid composition. ${ }^{15}$ Clinoptilolite and mordenite occur in the shallowest and coolest zones, and analcime or heulandite, and other less hydrated forms occur in the deeper and hotter zones. ${ }^{13,}{ }^{14}$ Submarine hydrothermal activity may be responsible for the formation of some zeolites in deepsea sediments.

Finally, burial digenesis, or metamorphism, can occur where zeolites associated with this system of formation are formed in thick volcanoclastic sediments that were metamorphosed at increased temperatures and pressures. ${ }^{14}$

\subsection{Synthetic Zeolites}

Hydrothermal zeolite synthesis typically proceeds from a reactive aluminosilicate gel at temperatures exceeding $90^{\circ} \mathrm{C}$ and autogenous pressures ${ }^{16-18}$, according to the scheme 
shown (Fig. 1.4). The mechanism of zeolite formation is actually very complicated, where each of the steps outlined in the figure involves a large number of reactive species with varying solubilities that serve as reagents for numerous polymerization-depolymerization and nucleation reactions. ${ }^{19}$ The synthesis of zeolites was first documented by St. Clair Deville ${ }^{10}$ in 1862 , where it was reported that by heating aqueous solutions of potassium silicate and sodium aluminate in a glass tube at $170^{\circ} \mathrm{C}$, produced levynite. In 1882 , De Schulten ${ }^{10}$ reported the synthesis of analcime. However, much of the work by the pioneers of zeolite synthesis cannot be reproduced in the laboratory, due to the unavailability of data from these researchers. ${ }^{20}$ Often overlooked is the fact that earlier work in turn depended on the first demonstration, by R. H. Milton of Union Carbide, USA in 1949, that zeolites could be synthesized using low temperature hydrothermal methods. At that time, major emphasis was placed on mimicking those conditions under which the zeolites of volcanic origin were presumed to have been formed, i.e. high temperatures and salt concentrations and autogenous pressures. $^{21}$

\subsubsection{Laboratory preparation}

A typical zeolite synthesis involves mixing together silicate and aluminate solutions (or sols) to form an aluminosilicate gel, usually instantaneously, which is then treated hydrothermally to give the crystalline product. ${ }^{22}$ The composition and structure of the aluminosilicate gel are of considerable interest; therefore, characterization of the aluminosilicate species present would give insight into the crystallization process. Silicate 
* Start

Si Source

Al Source

* Finish

Crystal

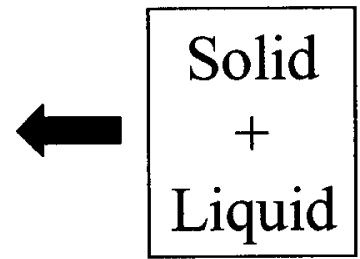

I

Soluble Si Species $+$

Soluble Al Species

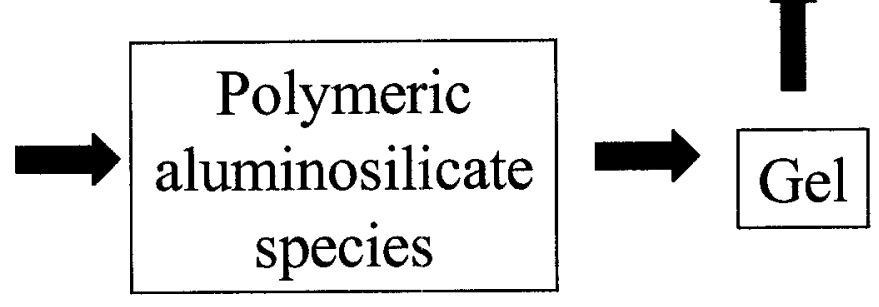

Fig 1.4 A simple scheme depicting hydrothermal zeolite synthesis from a reactive aluminosilicate gel at temperatures above $90^{\circ} \mathrm{C}$. 


\section{Chapter I General Introduction to Zeolites: Formation, Modification and Implication}

and aluminate solutions have been well studied so that one can be reasonably sure what species are present in a given solution of known concentration and $\mathrm{pH}^{22}$ Aluminate solutions have been shown to contain only one type of ion at high $\mathrm{pH}$; the tetrahedral $\mathrm{Al}(\mathrm{OH})_{4}^{-}$ion. ${ }^{23}$ At neutral $\mathrm{pH}$, various polymeric ions appear. With the lowering of $\mathrm{pH}$, more ions are formed, of which $\mathrm{Al}\left(\mathrm{H}_{2} \mathrm{O}\right)_{6}{ }^{3+}$ is predominant in these solutions. ${ }^{22}$ It is the tetrahedral aluminate ion that is the important species for zeolite synthesis, as $\mathrm{AlO}_{4}$ tetrahedra constitute as a basic building block. Silicate solutions, again at high $\mathrm{pH}$, contain a range of small silicate polymers, formed from corner-sharing tetrahedral $\mathrm{SiO}_{4}$ units. These polymers depolymerize quickly in response to increasing $\mathrm{pH}$ or dilution. ${ }^{22}$

\subsubsection{Commercial preparation}

The preparation of zeolites proceeds by simply loading a gel with components in prearranged proportions into sealed glass vials and then into a closed stainless steel 'bomb', or into a bomb lined with inert material, at the time of initial investigation of zeolite synthesis, was a dramatic change. Such bombs today are now commercially available (Fig. 1.5), and tuning of the temperature and pressure is routine. ${ }^{21}$

Current processes for manufacturing zeolites fall into one of three categories: (1) the hydrogel process, (2) clay conversion and (3) other processes. Tomlinson ${ }^{21}$ describes the hydrogel process as one that can be based either on homogeneous hydrogels, prepared from solutions of soluble reactants or heterogeneous solutions, which are prepared from reactive alumina or silica in a solid form. Typical gels are prepared from aqueous solutions of 


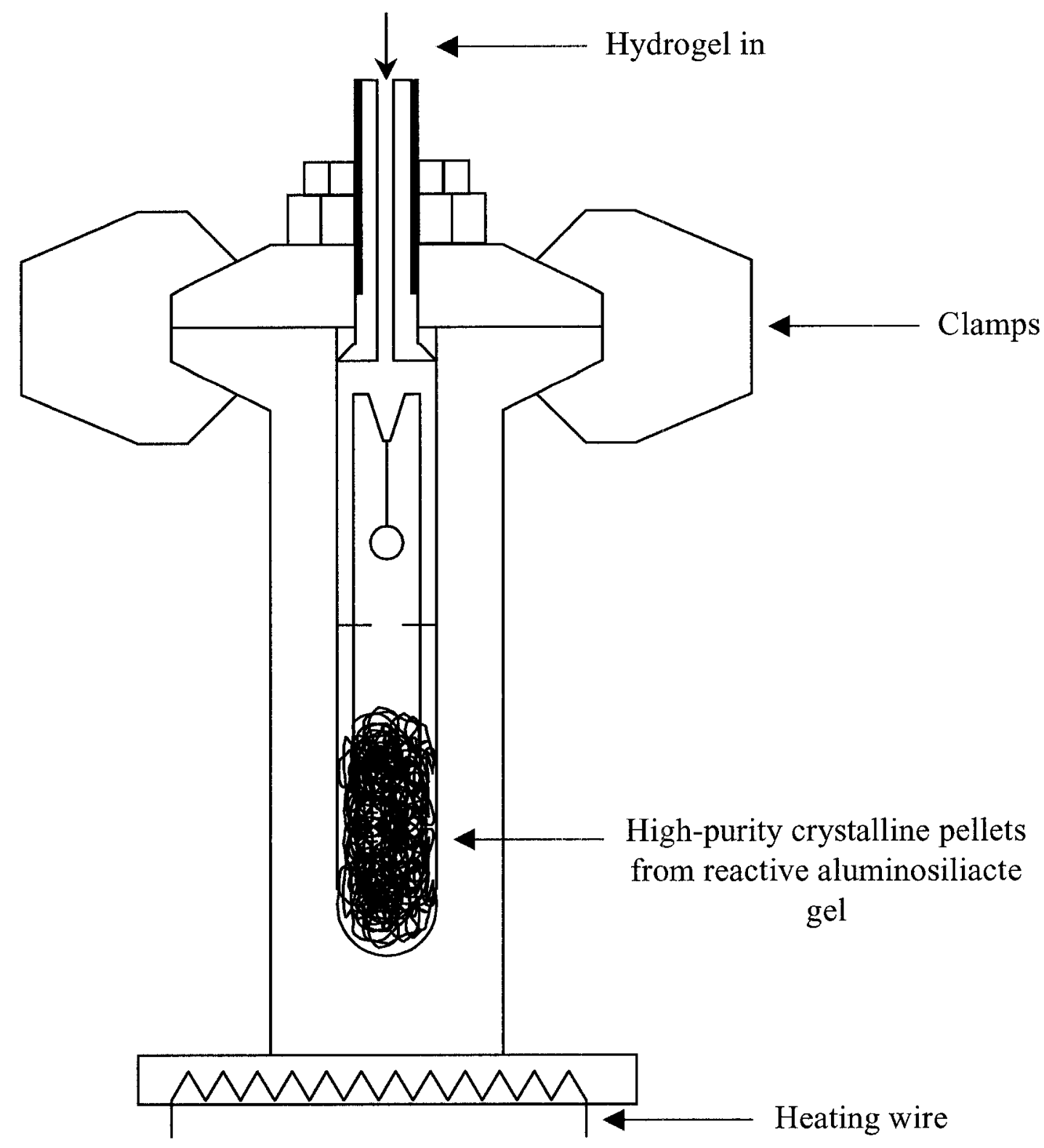

Fig. 1.5 A hydrothermal bomb in a configuration with removable internal attachments and a heating element for preparing giant zeolite crystals through a temperature gradient and nutrient. 
reagents such as sodium aluminate, sodium hydroxide and sodium silicate (Fig. 1.6), although other reagents can be used, including alumina trihydrate, colloidal silica and silicic acid. In the clay conversion process, the starting material is usually kaolin, which is first dehydrated three or four times to meta-kaolin by calcination in air (Fig. 1.7). Other processes involve either combinations of the first two processes, or a novel method altogether. One such novel method is called the Mizusawa process, which is unique in utilizing acid clay, a mineral occurring in Japanese deposits of montmorillonite. ${ }^{21}$

A new approach to zeolite synthesis was introduced by R. M. Milton and associates in the Union Carbide Corporation. The process started with very reactive components in closed systems and employed temperatures for crystallization which are more typical of the synthesis of organic compounds than they are of mineral formation. ${ }^{24,25}$ Many of the synthetic zeolites are formed at temperatures ranging from about room temperature to the boiling point of water. ${ }^{10}$

\subsection{Industrial applications of zeolites}

Whether the zeolite species be of natural or synthetic origin, immediate work was done to determine their potential use within industry. While zeolites in the realm of geology were mainly crystallographic marvels, chemists and engineers worked to introduce zeolites as catalysts and indispensable sorbents in industries such as oil-refining and detergent manufacture, and as cation exchangers in water purification plants. 
$\mathrm{NaOH}_{(\mathrm{aq})}+\mathrm{NaAl}(\mathrm{OH})_{4(\mathrm{aq})}+\mathrm{Na}_{2} \mathrm{SiO}_{3(\mathrm{aq})}$

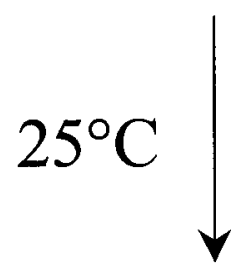

$\left[\mathrm{Na}_{a}\left(\mathrm{AlO}_{2}\right)_{b}\left(\mathrm{SiO}_{2}\right)_{c} \mathrm{NaOH} \mathrm{H} \mathrm{H}_{2} \mathrm{O}\right] \mathrm{gel}$

$25-175^{\circ} \mathrm{C} \mid$

Fig 1.6 A schematic representation of the preparation and crystallization of a $\mathrm{Na}_{2} \mathrm{O} \cdot \mathrm{Al}_{2} \mathrm{O}_{3} \cdot \mathrm{SiO}_{2} \cdot \mathrm{H}_{2} \mathrm{O}$ system gel. 


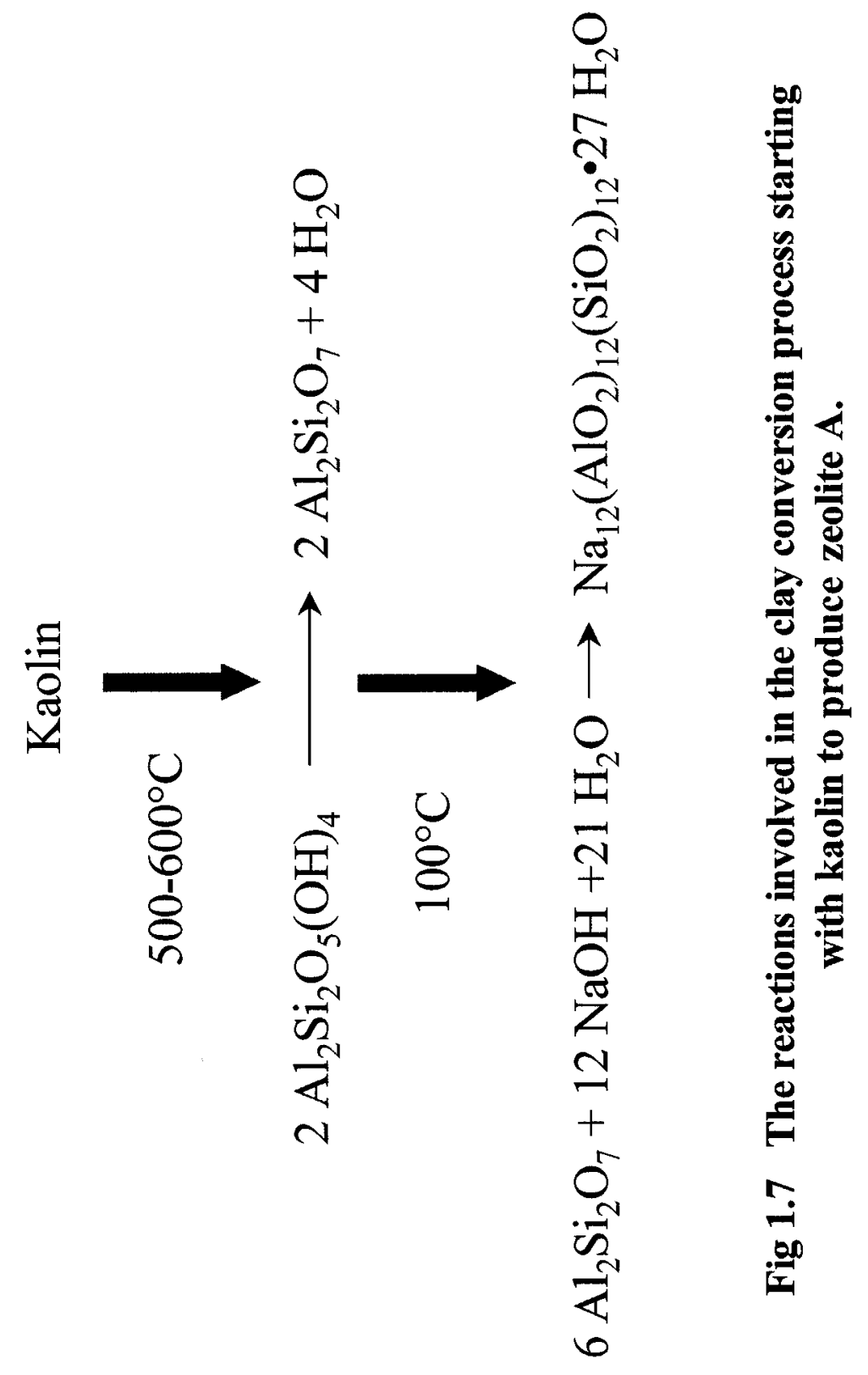




\subsubsection{Catalysis}

A catalyst is a substance that increases the rate of a reaction but is not itself consumed. Catalysts are widely used in nature, in industry, and in the laboratory, and it is estimated that they contribute to the majority of all manufactured goods in industrialized countries. ${ }^{26}$ Zeolites catalyse several types of reactions involving organic molecules. The most important are cracking, isomerization, and hydrocarbon synthesis, but there is an increasing application in the field of synthesis of fine organic chemicals. ${ }^{27}$ There are two fundamental considerations in zeolite catalysis: the first refers to the basic reaction mechanism; and the second to the way in which the products are controlled by the geometry and topology of the microporous crystal structure. ${ }^{27}$

The main reaction mechanism within zeolite catalysis is that of the Brønsted acid type, where the bridging hydroxyl groups are the most common type of acid site. They may be considered as protonated oxygens, the protons being present as charge compensators for the negatively charged framework aluminum. The coulombic interaction between the tetrahedral aluminum and the protons means that the $\mathrm{Si}-\mathrm{OH}-\mathrm{Al}$ bridges provide the dominant type of acid species, although acid sites associated with the defects in the zeolite structure are thought to contribute to catalytic processes. ${ }^{27}$ Depending on the preparation of the zeolite, the surface can consist of either Brønsted or Lewis acid character, or a combination of the two. ${ }^{28}$ Brønsted acid sites are converted into Lewis acid sites as the temperature is increased above approximately $500^{\circ} \mathrm{C}$ and water is driven off(Fig. 1.8). The strength of the 


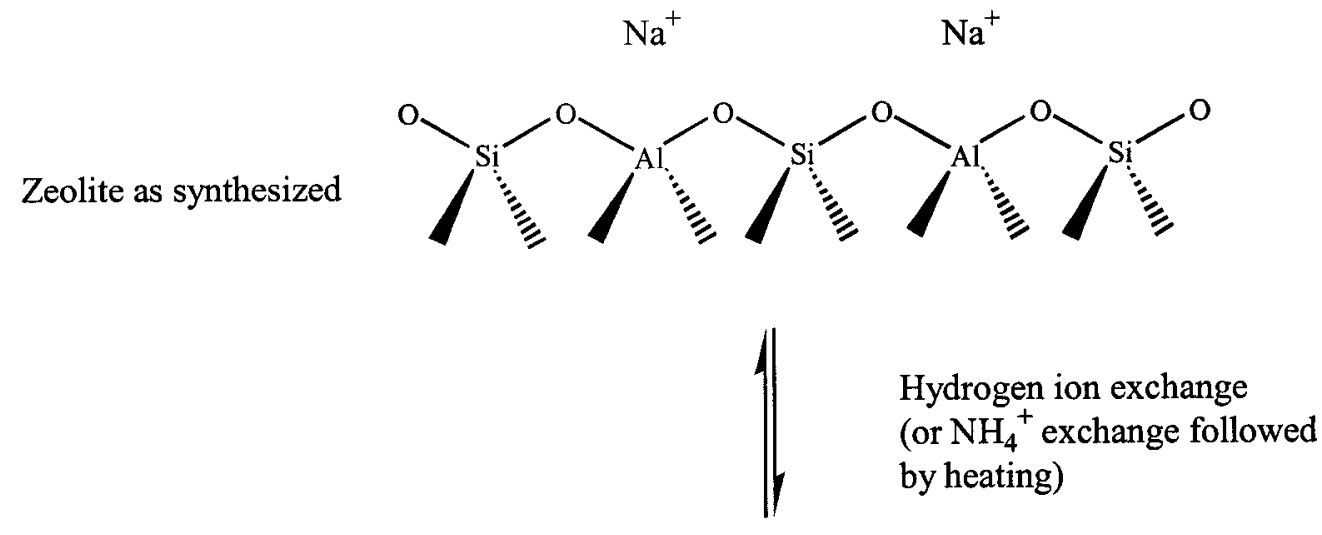

Bronsted acid form of zeolite<smiles>CC1(C)O[Si](C)(C)O[Si](C)(O[Si](C)(C)O[Si](C)(C)O)O1</smiles><smiles>C=CC=CCCCCCCO</smiles>

Lewis acid form of zeolite<smiles>CC(C)[Al](C)O[Si]1(C)C[C@H](C)O1</smiles>

Fig. 1.8 Generation of Bronsted and Lewis acid sites in zeolites. 
acid sites is related to the framework composition of the zeolite, where those with a high $\mathrm{Si}: \mathrm{Al}$ ratio have among the strongest acid sites. ${ }^{29}$

A special feature of zeolites which makes them such superb catalysts in some cases is their shape selectivity, which is manifested in three ways: (1) reactant selectivity, where only certain molecules may be absorbed into the zeolite cavities and thus reach the reactive sites; (2) product selectivity, derived from the fact that only certain products possess the correct dimension to escape the zeolite structure upon formation; and (3) the lesser important manner, transition state selectivity, where certain intermediates, which are formed during the reaction at the active site, will not fit in the cavity, thus limiting which reaction pathway will take place. $^{28}$

\subsubsection{Adsorption}

When water is removed from the voids created within the zeolite framework, the zeolite can then take in other molecules. This process is called sorption, and the zeolites are said to 'sorb' molecules into their void volume, that is, they act as sorbents. Frequently clathrate terminology is used, where the sorbing molecules are described as guests within the zeolite host. ${ }^{9}$ Generally, the $\mathrm{AlO}_{4}$ and $\mathrm{SiO}_{4}$ tetrahedra are linked together so as to form a ring of oxygen atoms. The oxygen windows having the greatest influence on sieving are those composed of 8,10 and 12 oxygens, i.e. the S8R, S10R and S12R rings. ${ }^{9}$ It should be appreciated that both synthetic and natural zeolites possess a wide variety of internal channels and cavities accessed by guest molecules through oxygen windows which happen 
to be close in size to the dimensions of common organic and inorganic molecules. ${ }^{9}$ Because of this inherent geometry, zeolites are then able to act as molecular sieves, separating mixtures of molecules in both liquid and gas phases on the basis of their effective sizes.

The use of zeolites as selective adsorbants is a recent and rather fascinating development. Unique geometries contained in the zeolitic channels and cavities result in selection sorption properties. These properties arise from the fact that not all oxygen windows are large enough to permit molecular progress through them. It is the smallest window, the S6R, that creates significant separations and is responsible for the selective sorption of water into the $\beta$ cages of several zeolite structures.

Table 1.2 shows examples that size is paramount in the selective properties of the zeolite. In the centre of the unit cell is a cavity which may be connected to identical cavities in adjacent unit cells by channels or windows. In the hydrated form, all of the cavities contain water molecules. In the anhydrous state, the same cavities may be occupied by other molecules brought into contact with the zeolite, provided those same molecules are able to squeeze through the apertures connecting cavities. The molecules are held by electrostatic and van der Waals forces. Thus, zeolites capture molecules that are just small enough to enter those cavities. They will not absorb molecules that are too large to enter, and will only weakly absorb very small molecules or atoms which can enter but leave easily. For example, zeolites may absorb straight-chain hydrocarbons but will not absorb branched alkanes or aromatic rings. 
Table 1.2 Some dimensional properties of common zeolites, where window size dictates whether molecules may be taken up by the zeolite structure.

\begin{tabular}{|c|c|c|c|c|}
\hline Zeolite & $\begin{array}{l}\text { Restricting } \\
\text { windows } \\
\text { (number of } \mathrm{O} \\
\text { atoms) }\end{array}$ & $\begin{array}{l}\text { Effective } \\
\text { window } \\
\operatorname{size}^{a}(\AA)\end{array}$ & $\begin{array}{c}\text { Void } \\
\text { volume }\end{array}$ & Other dimensions \\
\hline \multirow[t]{2}{*}{ Zeolite A } & 6 & 2.3 & \multirow[t]{2}{*}{0.47} & $\beta$ cage diameter: $6.6 \AA$ \\
\hline & 8 & 4.5 & & $\alpha$ cage diameter: $11.4 \AA$ \\
\hline \multirow[t]{2}{*}{ Zeolite X } & 6 & 2.3 & \multirow[t]{2}{*}{0.53} & \multirow[t]{2}{*}{$\alpha$ cage diameter: $11.8 \AA$} \\
\hline & 12 & 7.8 & & \\
\hline Zeolite L & 12 & 7.1 & 0.28 & \\
\hline \multirow[t]{2}{*}{ Heulandite } & 8 & $4.0 \times 5.5$ & \multirow[t]{2}{*}{0.35} & \multirow{2}{*}{ Interconnected } \\
\hline & 10 & $4.4 \times 7.2$ & & \\
\hline \multirow[t]{3}{*}{ Phillipsite } & 8 & $4.2 \times 4.4$ & \multirow[t]{3}{*}{0.30} & \multirow{3}{*}{ Interconnected } \\
\hline & 8 & $2.8 \times 4.8$ & & \\
\hline & 8 & 3.3 & & \\
\hline \multirow[t]{2}{*}{ Mordenite } & 12 & $6.7 \times 7.0$ & \multirow[t]{2}{*}{0.26} & \multirow{2}{*}{ Interconnected } \\
\hline & 8 & $2.9 \times 5.7$ & & \\
\hline
\end{tabular}

${ }^{a}$ May not be the crystallographic measurements.

${ }^{b}$ Expressed as $\mathrm{cm}^{3}$ liquid $\mathrm{H}_{2} \mathrm{O} / \mathrm{cm}^{3}$ crystal. 


\subsubsection{Ion exchange}

The zeolites function as cation exchangers, as observed when placing a zeolite in a solution containing a particular cation. An equilibrium occurs between the cation population outside and inside the zeolite. This equilibrium distribution is achieved rapidly since the porous structure allows rapid passage of ions in and out through the apertures of the zeolite cavities. In general, when there are two types of ions in solution, one sort will have a slightly greater preference for the zeolite environment than the other ion type. This results in a differential concentration between the aqueous phase and the zeolite environment. The naturally occurring zeolites were the first ion exchangers discovered, and industrially they are competing for this purpose with synthetic exchange resins which are designed for the same purpose.

Exchange between cation $A^{Z_{A}}$, initially in solution and $B^{Z_{B}}$, initially in a zeolite, can be written as follows: $Z_{B} A^{Z_{A}}+Z_{A} \bar{B}^{Z_{B}} \rightleftharpoons Z_{B} \bar{A}^{Z_{A}}+Z_{A} B^{Z_{B}}$, where $Z_{A, B}$ are the valencies of the ions and the characters designated with a bar related to a cation inside the zeolite crystal. Some simple exchanges are: $\mathrm{Na}^{+}+\overline{\mathrm{K}}^{+} \rightleftharpoons \overline{\mathrm{Na}}^{+}+\mathrm{K}^{+}$, for a uni-univalent exchange, and $2 \mathrm{Na}^{+}+{\overline{\mathrm{Cu}^{2}}}^{2} 2 \overline{\mathrm{Na}}^{+}+\mathrm{Cu}^{2+}$, a uni-divalent exchange. Cation exchange reactions can be characterized by ion exchange isotherms.

Hydrated cations within zeolite pores are loosely bound, and will readily exchange with other cations in surrounding aqueous media. ${ }^{17}$ The study of ion exchange is well established and the thermodynamics of ion exchange equilibria are well known. A 
traditional application is in water softening, and increasing use of zeolites is found in the detergent industry. Recent applications have concerned the removal of radioactive ions from contaminated water, particularly after the fission reactor incidents at both Three-Mile Island and Chernobyl. ${ }^{27}$

\subsection{Zeolites in Toxicology}

Because of their unique selective nature displayed in the fields of catalysis, adsorption and cation exchange, zeolitic molecular sieves have provided, since the very beginning of their commercialization, effective solutions for controlling pollution of the environment. ${ }^{30}$ The pressing need for environmental protection has given rise to research in the areas of green chemistry, solvent-free synthesis, zero emissions and the like. ${ }^{31}$ The aim is "production or reactor integrated environmental protection", which implies the use of better catalysts, the determination of thermodynamic and kinetic properties of the chemical reaction as well as the choice of the most suitable type of reactor and the optimal reaction conditions. ${ }^{31}$ In essence, the strategy has now become proactive as opposed to reactive.

As previously mentioned, zeolites find use as catalysts within the petroleum industry. The industrial production is mainly based on catalytic processes, and more than $90 \%$ of all chemical products manufactured involve at least one catalytic step, if not more, during a synthetic procedure. To this end, zeolites have the capability to selectively catalyze reactions that may require something either more exotic or harmful to the environment; some heavy metal catalysts come to mind. 
Zeolites with framework structures are excellent inorganic ion exchangers, having high stability to radioactivity. Akyil et. al. ${ }^{32}$ report that zeolite exchangers have been widely used in the full-scale separation of ${ }^{137} \mathrm{Cs}$ in waste solutions from nuclear power and fuel processing plants. Harjula and Lehto have studied the effect of sodium and potassium ions on cesium adsorption from nuclear power plant waste solutions on synthetic zeolites. ${ }^{33}$

It should be appreciated that zeolites in their natural or synthetic form can, with their inherent characteristic catalytic, ion-exchange and adsorbent behaviour be exploited for the benefit of industry and the environment altogether. As replacement catalysts in the catalytic cracking of hydrocarbons, in nuclear and municipal waste management for the exchange and removal of toxic heavy metals from water systems, zeolites that have been geologic mysteries until the present have found well-suited use.

\subsection{Zeolites studied}

In this study, surface modification and characterization of three zeolite types was done, specifically on the synthetic Linde Type A (LTA), of the $4 \AA$ and $5 \AA$ variety, and the natural zeolites, mordenite (MOR) and stilbite (STI). Linde zeolites were readily available in large quantities, in both varieties, and MOR and STI were provided from natural sources from The Bay of Fundy, Nova Scotia.

\subsubsection{Linde Type A (LTA)}

Milton and Breck of the Linde division of Union Carbide, over a period of 5 years, developed and characterized three novel zeolites, Linde $\mathrm{A}, \mathrm{X}$ and $\mathrm{Y}$, which have become 
three of the most profitable synthetic zeolites for industry. In their attempts to readily synthesize chabazite in 1949, they accidentally stumbled across new structures, zeolites A and $\mathrm{B}$, or gismondite, zeolite $\mathrm{C}$, or sodalite, and a crystalline impurity named zeolite $\mathrm{X}^{34}$ Experimentation came with changing traditional approaches to zeolite synthesis, specifically hydrothermal syntheses, where relatively insoluble forms of silica and alumina were placed in mildly alkaline solutions ( $\mathrm{pH} 8-11$ ), containing calcium, magnesium and sodium cations at temperatures of $200-300^{\circ} \mathrm{C}$. Reaction periods varied from a few days to a week; in some experiments there was no reaction, in others analcime or small-pore mordenite was produced. It was then attempted to increase the pressure within the reaction vessel to $2000 \mathrm{psig}$, but it was later concluded that this had no effect on zeolite crystallization. Adsorption properties of zeolite A were determined, and followed the discovery that the pore size could be reduced or enlarged by appropriate choice of potassium, calcium or magnesium ions. ${ }^{34}$ It was also noted that the zeolite was stable at $500^{\circ} \mathrm{C}$. Milton and Breck intended for the synthesized zeolites to have some sort of use, so the researchers postulated that zeolites A and X should make good catalysts or catalyst supports, specifically with respect to hydrocarbon cracking, abnormally strong adsorption sites, molecular size selectivity, and the possibility of atomically dispersed metals on the internal surfaces. ${ }^{34}$

In crystallographic terms, LTA can be characterized by its secondary building units: S4R, and D4R, 8-rings or 6-2. Depending on the cation that was present during synthesis, the aperture size can vary. Those being used in this study are of the $4 \AA$ and the $5 \AA$ aperture 
size, containing sodium and calcium cations within their structure, respectively. The unit cell for LTA can be seen in Fig. 1.9, which shows the view along the [100] plane. LTA has a general formula of $\left\{\mathrm{Na}_{12}\left[\mathrm{Al}_{12} \mathrm{Si}_{12} \mathrm{O}_{48}\right] \cdot 27 \mathrm{H}_{2} \mathrm{O}\right\}_{8}$, and crystallizes in the cubic space group Fm $\overline{3} \mathrm{c}$, with unit cell parameter, $a=24.6 \AA .^{11}$

\subsubsection{Mordenite (MOR)}

Synthesis of mordenite was first reported by Barrer and then by many successors. ${ }^{35}$ The structure of mordenite was determined by Meier ${ }^{36}$ and consists of an orthorhombic structure containing chains of 5-membered rings parallel to the $c$-axis connected through double 8-membered rings in the $a-c$-plane. These chains join to give 12-ring channels in the $c$-direction, with double 8-ring prisms lining the channel in the $a$-c-plane. As adjacent 8-ring prisms lining are offset about $3.7 \AA$ in the $c$-direction (c/2), the path between the 12-ring channels, with dimensions, $6.5 \AA$ by $7.0 \AA$, is a very distorted 8 -ring having a minimum free diameter of about $2.8 \AA$ (Fig. 1.10). Diffusion, in three dimensions, is therefore limited to very small molecules or cations.

Mordenite is one of the best known acid zeolites, with a well understood pore chemistry. It acts as a catalyst in protonation reactions of alkanes, alkenes and aromatic ring compounds, which require Brønsted acid sites. A typical example is the protonation of adsorbed terpenes, where electron paramagnetic resonance techniques have demonstrated that stable carbocations are formed. ${ }^{37}$ The thermal stability of MOR due to the high Si/Al ratio makes it applicable in thermal swing adsorption processes where high temperature is 


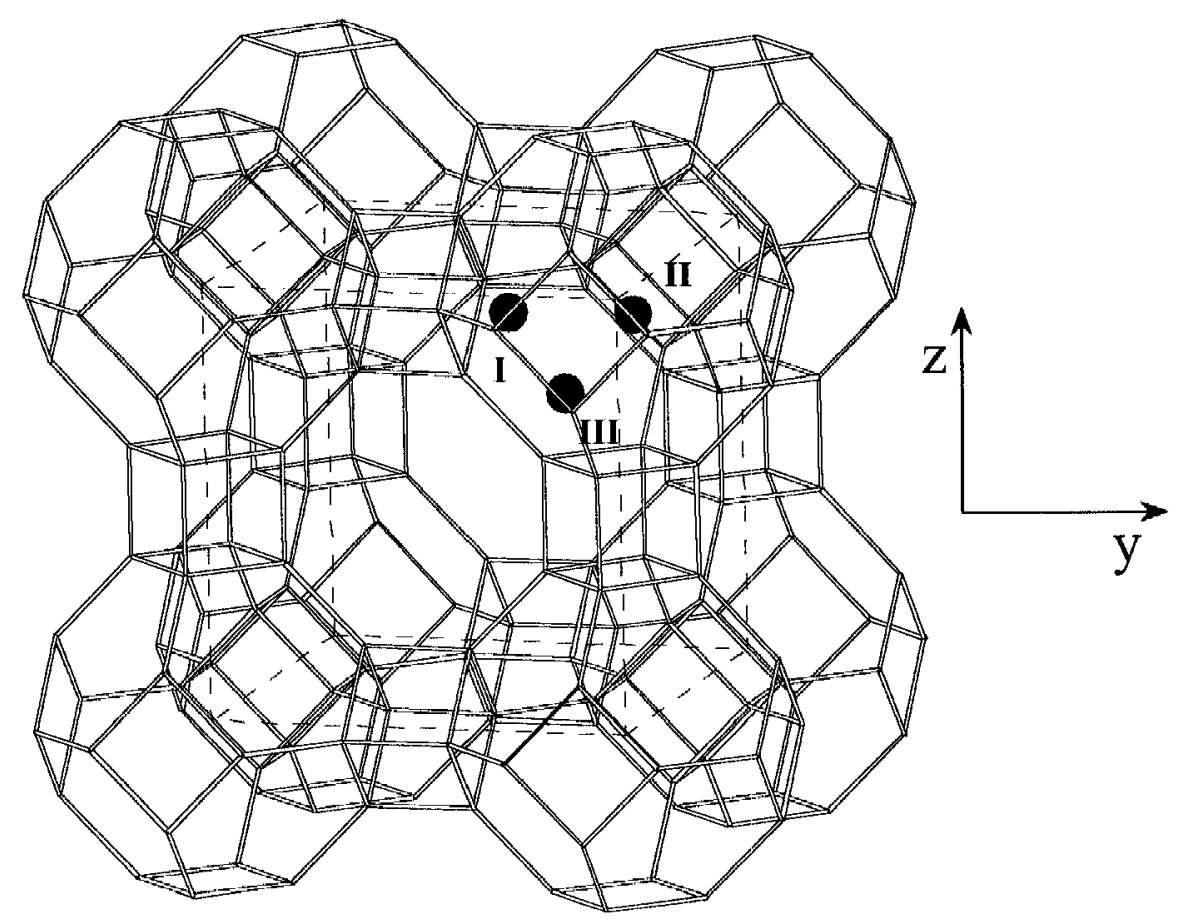

Fig. 1.9 Framework of LTA viewed along [100]. Blue spheres indicate cation positions within the framework structure. 


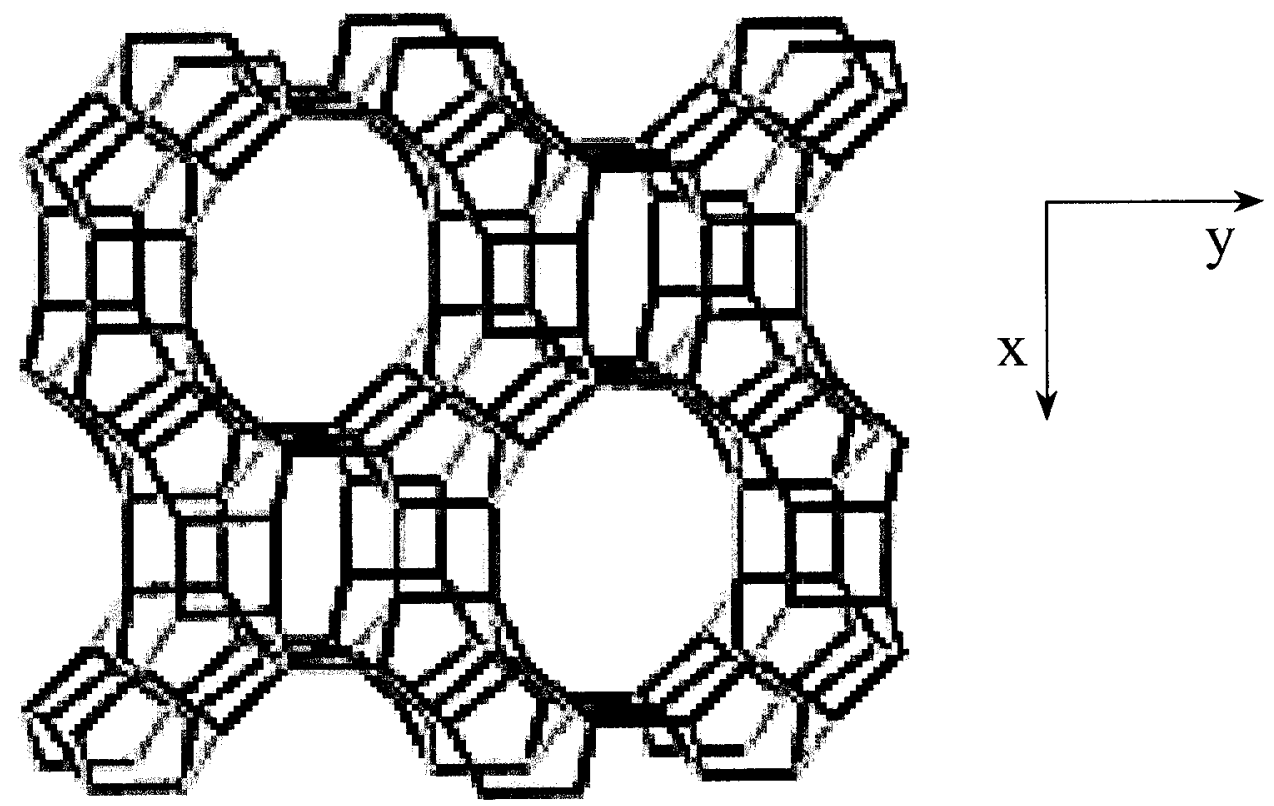

Fig. 1.10 Framework of MOR viewed along [001]. 
required for desorption. ${ }^{38}$ Not only the peculiar skeleton and pore structures of MOR, but also the arrangement of cationic species and positions have an important role in its application to adsorption and separation. ${ }^{38}$ Mordenite is one of the more siliceous zeolites, with an idealized composition: $\mathrm{Na}_{8} \mathrm{Al}_{8} \mathrm{Si}_{40} \mathrm{O}_{96} \cdot 24 \mathrm{H}_{2} \mathrm{O}$.

\subsubsection{Stilbite (STI)}

Stilbite, which is commonly found as a vein mineral in volcanogenic rock, is associated with minerals of the heulandite group. Stilbite has been investigated in different countries by several workers. The crystal structure of STI has been reported by Gottardi, Slaughter and later refined by Galli. ${ }^{39}$ They report the crystal structure of stilbite is characterized by large, intersecting, relatively open channels of 10- and 8-membered tetrahedral rings (Fig. 1.11). The 10-membered rings have dimensions of $4.9 \AA \times 6.1 \AA$ in the [100] plane that are interconnected to the channels formed by the 8-membered rings in the [101] plane that have dimensions $2.7 \AA \times 5.6 \AA .{ }^{11}$ STI belongs to the monoclinic space group, with $\mathrm{C} 2 / \mathrm{m}$, with unit cell parameters, $a=13.6, b=18.2$ and $c=17.8 \AA$, and $\beta=91^{\circ}$. STI is a silica-rich zeolite, and could be potentially used as an adsorbent and catalyst, with idealized composition $\mathrm{Na}_{4} \mathrm{Ca}_{8}\left[\mathrm{Al}_{20} \mathrm{Si}_{52} \mathrm{O}_{144}\right] \cdot 56 \mathrm{H}_{2} \mathrm{O}$. However, due to easy collapse of the framework during dehydration at elevated temperature, makes use of this zeolite difficult. The collapse is caused by cell contraction of the stilbite structure. ${ }^{40}$

\subsection{Limitations of current zeolite knowledge}

Everything said thus far concerned the structures of zeolites, their uses, properties, 


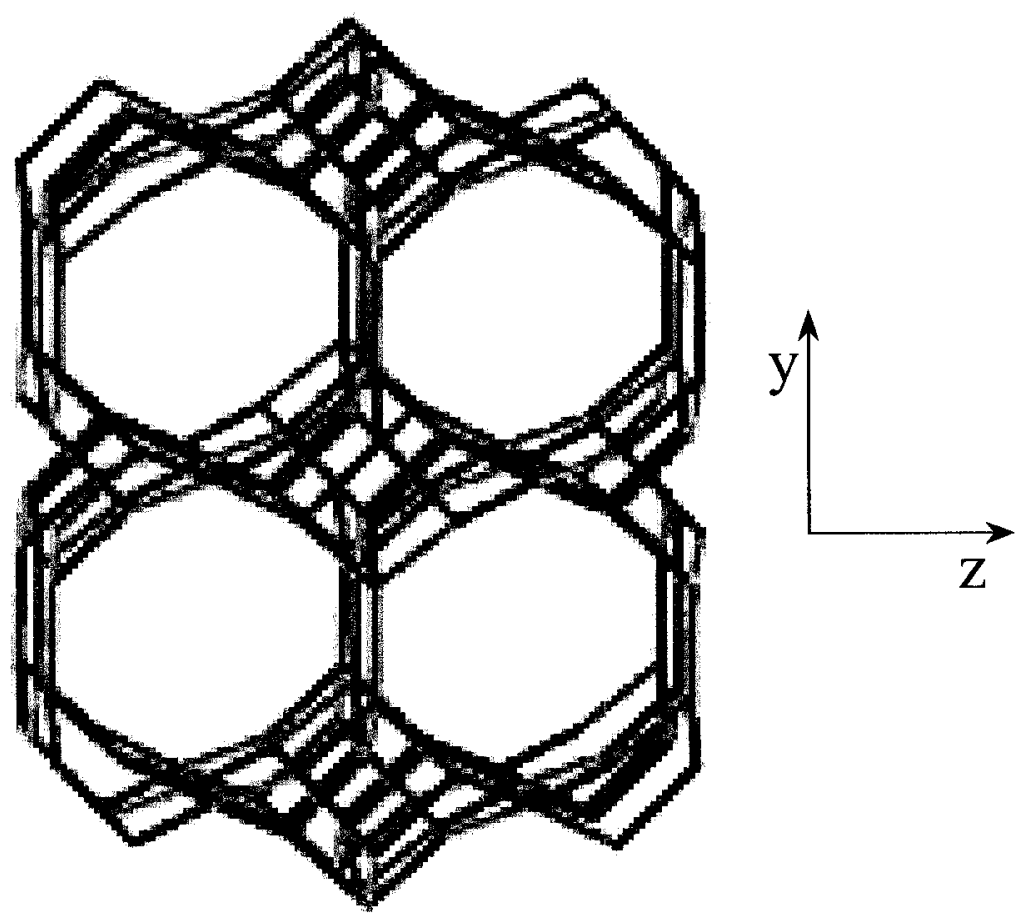

Fig. 1.11 Framework of STI viewed along [100]. 
synthetic and natural origins; this knowledge is accessible in all classic textbooks. A relatively unexplored area of zeolite literature is the study of the chemically modified surfaces of zeolites, and the effect on the adsorptive nature of the zeolite subsequent to surface modification. It is intended through the studies shown in this thesis, to increase the current knowledge of the effect of the modification of zeolite surfaces, and to study the change in one of the intrinsic characteristics of zeolites: their capability to adsorb water from the atmosphere.

\subsection{Thesis overview}

Based on previous work done with coating of glass surfaces ${ }^{41}$, and knowing the chemistry involved in the attachment of covalently-bound organosilanes to those surfaces, it was demonstrated that this could be generalized to the modification of zeolite surfaces. By exploiting the available surface hydroxyls that exist on zeolite surfaces, covalent linkages result in the formation of Si-O-Si covalent bonds via the introduction of organosilane materials that contain a chlorine functionality.

The zeolites that are studied here are the synthetic Linde $4 \AA$ and $5 \AA$ varieties and the natural zeolites stilbite (STI) and mordenite (MOR). Because it is known that most zeolites are hydrophilic materials, it is thought that this hydrophilicity could be controlled in some manner, i.e. inhibited by a hydrophobic coating agent such as an organosilane. The organosilane chosen possesses a chlorine functionality that is expected to react with the surface hydroxyls of the zeolite, where the organic moiety varies in chain length and degree 
of branching. This coating capability has been observed and exploited in the coating of glass, where the surface hydroxyls of the glass are available for bonding with the chlorine of the organosilane. Once the coating material is introduced to the zeolite, the interaction results in a covalent linkage between the coating agent and the zeolite, providing the zeolite with a layer of intrinsically hydrophobic material above the zeolite, inhibiting the sorption of water by the zeolite. This protective layer then allows the zeolite to adsorb organic molecules into its structure that it would not normally take up as a result of the water molecules that would rush into the cavities taking up all available space.

Of all the organosilane agents that could be used, the ones chosen for this study are the commercially available SurfaSil ${ }^{\mathrm{TM}}$ (SS), trimethylchlorosilane (TMCS) and a newly synthesized pyrene derivative, pyrene-1-(4-butyl-dimethylchlorosilyl) ether (Pyrene-Cl).

In addition to the manipulation of this hydrophilic/hydrophobic behaviour, the addition of the organic layer also allows the zeolite to behave as a molecular sieve in another way than just the traditional manner where the cavity size controls the sieving behaviour, as in the instance of cation exchange. To this end, it was proposed that the organic layer on the surface of the zeolite could not only behave as a "protector" from atmospheric water entering the zeolite structure, but also as a filter for organic materials that might also be present. By controlling the branching nature of polarity of the zeolite coating, one can tailor the sieving behaviour to a desired compound to be filtered out from a mixture of compounds.

The synthesis of the pyrene derivative shows the potential for a "trap door" 
mechanism, where the pyrene moiety is able to behave as a door that could possess the ability to trap substances within the zeolite pore structure by increasing or decreasing the temperature, giving rise to a novel mechanism for gas storage and transport, perhaps eventually eliminating the need for pressurized gas cylinders altogether.

Overall, this project entails the modification and characterization of both synthetic and natural zeolites. Using various analytical and spectroscopic techniques allows for the determination of degree of coating, amount of water loss and gain, surface area, rate of water adsorption and structure analysis. Through the introduction of readily available and newly synthesized organosilane coatings, a new use for zeolites has been introduced, and a novel method of molecular sieving and potential solvent purification and gas storage/transport is envisioned. 


\section{References}

1. Gottardi, G. and E. Galli, Natural Zeolites. 1985: Springer-Verlag Berlin Heidelberg. 409.

2. Cotton, A.F., G. Wilkinson, C.A. Murillo, and M. Bochmann, Advanced Inorganic Chemistry. 6th ed. 1999: John Wiley \& Sons, Inc.

3. Way, J.T., J. R. Agric. Soc., 11, 1850, 313-379.

4. Breck, D.W., Soc. Min. Eng., AIME 73H36, 1973, 32.

5. Weigel, O. and E. Stinhoff, Z. Kristallogr., 61, 1925, 125-154.

6. Breck, D.W., J. Chem. Educ., 41, (12), 1964, 678-689.

7. Barrer, R.M., Zeolites and Clay Minerals as Sorbents and Molecular Sieves. 1978: Academic. 499.

8. Barrer, R.M. Zeolite Synthesis: Some Chemical Aspects. in Proceedings of Sixth International Zeolite Conference. 1983. Reno, NV: Butterworth.

9. Dyer, A., An Introduction to Zeolite Molecular Sieves. 1988: John Wiley \& Sons. 149.

10. Breck, D.W., Zeolite Molecular Sieves: Structure, Chemistry and Use. 1974, New York: John Wiley \& Sons, Inc. 771.

11. Baerlocher, C., W.M. Meier, and D.H. Olson, Atlas of Zeolite Framework Types. 5th Revised ed. 2001: Elsevier.

12. Breck, D.W., Recent Advances in Zeolite Science, in Molecular Sieve Zeolites-I, R.F. Gould, Editor. Vol. 101. 1971, ACS. p. 1-17.

13. Hay, R.L., Geology of Zeolites in Sedimentary Rocks, in Mineralology and Geology of Natural Zeolites, F.A. Mumpton, Editor. Vol. 4. 1981, Bookcrafters, Inc.: Chelsea, Michigan.

14. Hay, R.L., Geologic Occurrence of Zeolites, in Natural Zeolites: Occurrence, Properties and Use, L.B. Sand and F.A. Mumpton, Editors. 1978, Pergamon. p. 
$135-143$.

15. Iijima, A. Geology of Natural Zeolites and Zeolitic Rocks. in Proceedings of the Fifth International Conference on Zeolites. 1980. Naples Italy: Heyden.

16. Barrer, R.M., Hydrothermal Chemistry of Zeolites. 1982, London: Academic Press.

17. Breck, D.W., Zeolite Molecular Sieves: Structure, Chemistry and Use. 1984, Florida: Krieger.

18. Szostak, R., Molecular Sieves: Principles of Synthesis and Identification. 1989, New York: Van Nostrand Reinhold.

19. Dutta, P.K. and J. Bronić Zeolites, 14, 1994, 250.

20. Clifton, R.A., Natural and Synthetic Zeolites, Bureau of Mines, 1987, IC 9140.

21. Tomlinson, A.A.G., Modern Zeolites: Structure and Function in Detergents and Petrochemicals. 1998, Switzerland: Trans Tech Publications Ltd. 82.

22. Harvey, G. and L.S.D. Glasser, Structure and Properties of Aluminosilicate Solutions and Gels, in Zeolite Synthesis, M.L. Occelli and H.E. Robson, Editors. 1989, American Chemical Society: USA. p. 49-65.

23. Akitt, J.W., N.W. Greenwood, B.L. Khandelwal, and G.D. Lester, J. Chem. Soc. Dalton Trans., 1972, 604.

24. Milton, R.M., U.S. Patent, 2,882,243, 1959.

25. Milton, R.M., U.S. Patent, 2,882,244, 1959.

26. Shriver, D. and P. Atkins, Inorganic Chemistry. 3rd ed. 1999, New York: W. H. Freeman and Company.

27. Catlow, C.R.A., Zeolites: Synthesis, Structure and Properties - An Introduction, in Modelling of Structure and Reactivity in Zeolites, C.R.A. Catlow, Editor. 1992, Academic Press: San Diego.

28. Clark, J.H. and C.N. Rhodes, Clean Synthesis using Porous Inorganic Solid 
Catalysts and Supported Reagents. RSC Clean Technology Monographs, ed. J.H. Clark. 2000, Cambridge: The Royal Society of Chemistry.

29. Thomas, J.M., Sci. Amer., 82, 1992.

30. Fajula, F. and D. Plee, Advanced Zeolite Science and Applications, 85, 1994.

31. Hölderich, W.F., J. Röseler, G. Heitmann, and A.T. Liebens, Catalysis Today, 37, 1997, 353-366.

32. Akyil, S., M.A.A. Aslani, and S.O. Aytas, Journal of Alloys and Compounds, 271-273, 1998, 769-773.

33. Harjula, R. and J. Lehto, Nucl. Chem. Waste Management, 6, 1986, 133.

34. Milton, R.M., Molecular Sieve Science and Technology, A historical perspective, in Zeolite Synthesis, M.L. Occelli and H.E. Robson, Editors. 1989, American Chemical Society: Washington D. C.

35. Sun, Y., T. Song, S. Qiu, W. Pang, J. Shen, D. Jiang, and Y. Yue, Zeolites, 15, $1995,745$.

36. Meier, W.M., Z. Kristallogr., 115, 1961, 439.

37. Glod, B.K., G. Perez, and A.A.G. Tomlinson, Journal of Chromatography A, 760, 1997, 292.

38. Wang, Z.-M., T. Arai, and M. Kumagai, Ind. Eng. Chem. Res., 40, 2001, 1864.

39. Kasture, M.W., S.P. Mrajakar, V.V. Joshi, A.L. Choudhari, and V.P. Shiralkar, Journal of Inclusion Phenomena and Molecular Recognition in Chemistry, 29, 1997, 1.

40. Li, J., J. Qiu, Y. Sun, and Y. Long, Microporous and Mesoporous Materials, 37, 2000, 365 .

41. Breeze, S.R., S. Lang, I. Moudrakovski, C.I. Ratcliffe, J.A. Ripmeester, G. Santyr, B. Simard, and I. Zuger, Journal of Applied Physics, 87, 2000, 8013. 


\section{Chapter II}

\section{Experimental Techniques}

1. Introduction $\ldots \ldots \ldots \ldots \ldots \ldots \ldots \ldots \ldots \ldots \ldots \ldots \ldots \ldots \ldots \ldots \ldots \ldots$

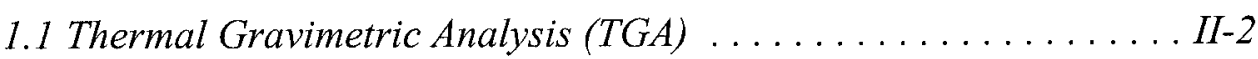

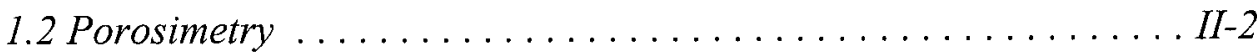

1.3 Powder X-ray Diffraction (PXRD) $\ldots \ldots \ldots \ldots \ldots \ldots \ldots$

1.4 Solid-state Nuclear Magnetic Resonance Spectroscopy (SS NMR) . II-4

1.4.1 ${ }^{29}$ Si NMR . . . . . . . . . . . . . . . . II-6

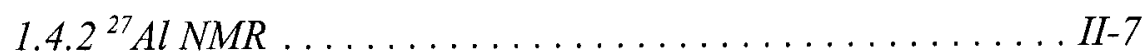

2. Experimental methods . .................II-10

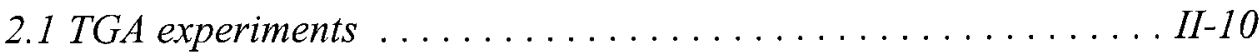

2.1.1 Sample weight loss measurements . . . . . . . . . II-10

2.1 .2 Temperature cycle experiments $\ldots \ldots \ldots \ldots \ldots \ldots$ II-10

2.2 Porosimetry measurements . . . . . . . . . . . . II-11

2.3 PXRD measurements $\ldots \ldots \ldots \ldots \ldots \ldots \ldots \ldots \ldots \ldots \ldots \ldots$

2.4 SS MAS NMR spectroscopy methods . . . . . . . . . . . II-11

$2.4 .1{ }^{13} \mathrm{C} S S$ MAS NMR experiments . . . . . . . . . II-11

2.4.2 ${ }^{29}$ Si SS MAS NMR experiments . . . . . . . . . II-11

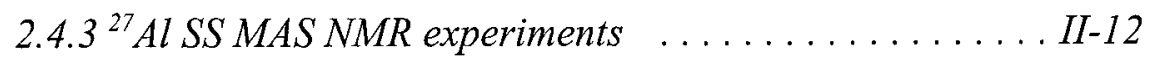

2.5 General procedure for the coating of zeolites $\ldots \ldots \ldots \ldots I I-12$

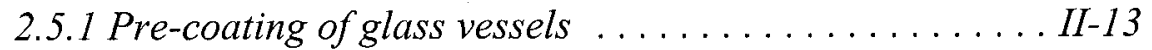

2.6 General Pyrene-derivative Synthetic Procedure . . . . . . . . . II-13

2.6.1 Synthesis of Pyrene-TBDMS . . . . . . . . . II-14

2.6.2 Synthesis of Red-Pyrene-TBDMS . . . . . . . .II-14

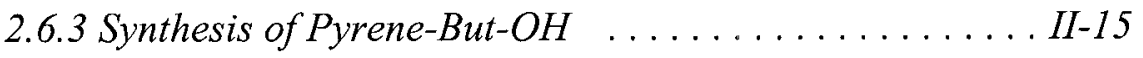

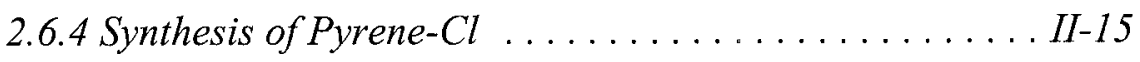

2.7 Adsorption experiment setup $\ldots \ldots \ldots \ldots \ldots \ldots \ldots \ldots \ldots$ II-16

References ........................II-17 


\section{Chapter II Experimental Techniques}

\section{Introduction}

This chapter outlines the physical techniques to be employed for the characterization of the neat and modified zeolite samples in this study. Specifically, thermal gravimetric analysis (TGA) was employed for the observation of weight loss or gain by the zeolite due to the effects of the coating material covalently bound to the zeolite surface. Powder X-ray diffraction (PXRD) chiefly was used for the confirmation of crystal structure of both the first natural sample (NS1), thought to be mordenite and the second natural sample(NS2), thought to be stilbite. ${ }^{13} \mathrm{C}$ solid-state magic angle spinning nuclear magnetic spectroscopy (SS MAS NMR) determined the existence of methyl carbons from the coating material; ${ }^{29} \mathrm{Si}$ and ${ }^{27} \mathrm{Al}$ SS MAS NMR were used to look at the local nuclear environments within NS1 and NS2 crystal structures, again, to determine sample identity.

\subsection{Thermal Gravimetric Analysis (TGA)}

The technique of thermal gravimetric analysis is used to measure the amount and rate of weight change in a material, either as a function of increasing temperature, or isothermally as a function of time, in a controlled atmosphere. It can be used to characterize any material that exhibits a weight change and to detect phase changes due to decomposition, oxidation, or dehydration. The derived percent weight change helps to identify and correlate chemical structure, processing, and end-use performance. ${ }^{1}$

\subsection{Porosimetry}

The study of the determination of sample pore size, surface area and isothermal 
adsorption of gas by a zeolite will be, in this work, referred to as the technique of porosimetry. The growing importance of adsorption has resulted in the appearance of an ever increasing volume of scientific and technical literature on novel adsorbents and catalysts. Also, various new procedures have been introduced over the past few years for the interpretation of adsorption data - particularly surface area determination, micropore and mesopore analysis. $^{2}$

\subsection{Powder X-ray Diffraction (PXRD)}

An X-ray powder diffraction pattern is a set of lines or peaks, each of different intensity and position that can be calculated using Bragg's Law: $\mathrm{n} \lambda=2 \mathrm{~d} \sin \theta$, where $\mathrm{d}$ spacing or Bragg angle, $\theta$, for a given substance describe the line positions that are essentially fixed and are characteristic of that substance. The line intensities may vary somewhat from sample to sample, depending on the method of sample preparation and the instrumental conditions. For identification purposes, principal note is taken of line positions together with a semi-quantitative consideration of intensities. ${ }^{3}$ Powder diffraction techniques are used to identify a sample of a solid substance by comparison of the positions of the diffraction peaks and their intensities with a large data bank, The Powder Diffraction File, which is maintained by the Joint Committee on Powder Diffraction Standards (JCPDS), and contains information on over 30000 substances. The technique is also used for the initial determination of the dimensions and symmetries of unit cells. ${ }^{4}$ 


\subsection{Solid-state Nuclear Magnetic Resonance Spectroscopy (SS NMR)}

Most NMR spectroscopy is performed on fluid samples, specifically solutions. Where solid materials are to be analysed using this non-destructive evaluation technique, a different method is required. NMR is a spectroscopic technique based on the phenomenon that some atomic nuclei possess a spin which interacts with an applied magnetic field, $B_{0}$. For a nucleus with spin quantum number $I$, this Zeeman interaction gives rise to $2 I+I$ separate energy levels between which transitions can be induced by radiofrequency irradiation. The Zeeman interaction usually is the greatest interaction, but not the only one. ${ }^{5}$ Other interactions which contribute to the appearance of the NMR spectrum are (1) the dipolar interaction, $H_{d}$, defined as the interaction of the spin with other spins; (2) the chemical shift interaction, $H_{c s}$, the interaction of the spin with the electrons in the electron cloud around the nucleus; (3) the quadrupole interaction, $H_{q}$, which is the interaction of the quadrupole moment of the nucleus with the electric field gradient at the nuclear site (where $I>1 / 2)$.

An essential difference between NMR of fluids and solids is the anisotropic spin interactions that occur in a solid sample, whereas these interactions are averaged out in a fluid by rapid tumbling of the molecules. For a solid sample, this random tumbling does not occur and the anisotropic spin interactions yield NMR frequencies which depend on the orientation of the magnetic field with respect to the molecular or crystal axes. ${ }^{5}$

For powders, such as zeolites, all possible orientations of crystallites are present, 
which may lead to broad and featureless resonances in the NMR spectrum. The anisotropy of the chemical shift is removed by a technique called magic angle spinning (MAS). In this technique, the sample is spun around an axis which is inclined at an angle of $54.74^{\circ}$ to the main static magnetic field, so that all tensor interactions within the sample are scaled down to zero to yield clean spectra with sharp lines. ${ }^{6}$

While MAS leads to line-narrowing and thus increases the sensitivity as well as resolution, cross-polarization (CP) can be used to further enhance the sensitivity of a nucleus with a low gyromagnetic ratio.? The process begins with the generation of transverse magnetization from the more sensitive spin species. This magnetization can then be "spinlocked" along an rf-field, $B_{l}$, that precesses in phase with it in the transverse plane. Because the $B_{l}$ field is much smaller than the external field, $B_{0}$, which was the original field for establishing magnetization, the spin-locked magnetization is in a non-equilibrium state. ${ }^{7}$ If an rf-field of the appropriate amplitude, is simultaneously applied to the other spin species, energy-conserving magnetization transfer can occur between the two types of spins, leading to the enhancement of the signal of the less-sensitive spins. ${ }^{7}$ CP has been widely applied to many inorganic materials and is frequently combined with MAS to yield well-resolved spectra with a good signal-to-noise ratio. It is particularly useful for enhancing the spectra of nuclei with a low natural abundance, which otherwise would be difficult to observe. Although CP MAS has most commonly been used with spin- $1 / 2$ nuclei, it has been applied to quadrupolar nuclei as well. ${ }^{7}$ 
It is the technique of CP MAS that is used in this study, specifically ${ }^{13} \mathrm{C}$ SS NMR to show the presence of coating materials used to modify the zeolite surfaces, and ${ }^{29} \mathrm{Si}$ and ${ }^{27} \mathrm{Al}$ nuclei for the characterization of NS1 and NS2. The details of ${ }^{29} \mathrm{Si}$ and ${ }^{27} \mathrm{Al}$ SS NMR techniques are described below.

\subsection{1 ${ }^{29} \mathrm{Si}$ NMR}

Silicon has only one naturally-occurring isotope with non-zero spin, namely ${ }^{29} \mathrm{Si}$, which has spin $I=1 / 2$ and a natural abundance of $4.70 \%$. This places it among the category of "rare" spins, and implies that all the modern techniques can be applied in a similar fashion to their use for ${ }^{13} \mathrm{C}$, for example. A number of review papers have been published ${ }^{8}$ summarizing the structural information and relationships available through the ${ }^{29} \mathrm{Si} \mathrm{NMR}$ on zeolites. One of the most important results of Lippmaa, Engelhardt and coworkers ${ }^{91}{ }^{10}$ was to establish the relationship between the ${ }^{29} \mathrm{Si}$ chemical shift sensitivity and its surrounding environment: the number and type of tetrahedrally coordinated atoms (T-atoms, with $\mathrm{T}=\mathrm{Si}$, $\mathrm{Al}$ or other lattice atoms) connected to a given $\mathrm{SiO}_{4}$ unit. The degree of connectivity is symbolized by $\operatorname{Si}(n \mathrm{Al})$ where $n=0,1,2,3$ or 4 , where $n$ indicates the number of Al atoms sharing oxygens with the $\mathrm{SiO}_{4}$ tetrahedron under consideration. Furthermore, the ${ }^{29} \mathrm{Si}$ chemical shift is influenced by the Si-O-T bond angle, and $\mathrm{Si}-\mathrm{O}$ bond length, which means that chemically equivalent but crystallographically inequivalent Si nuclei may have different chemical shifts. ${ }^{11}$ Chemical shift ranges are given in Fig. 2.1. Generally, the ${ }^{29}$ Si chemical resonance shifts to lower field by approximately $5 \mathrm{ppm}$ per additional aluminum. Therefore, 
up to five lines per crystallographically inequivalent Si site may be observed in the ${ }^{29} \mathrm{Si}$ NMR spectrum. Hence, useful information about the local environment of the $\mathrm{SiO}_{4}$ tetrahedra forming the zeolite lattice can be ascertained from the ${ }^{29} \mathrm{Si}$ chemical shift data. ${ }^{12}$

\subsection{2 ${ }^{27} A l N M R$}

The main applications of ${ }^{27} \mathrm{Al}$ NMR spectroscopy in connection with zeolites has been in the monitoring of dealumination processes and the detection of extra-framework aluminum, since extra-lattice aluminum is octahedrally coordinated and gives rise to resonance lines at about $0 \mathrm{ppm}$, whereas framework aluminum is tetrahedrally coordinated and resonates between approximately 50 and 65 ppm. ${ }^{8}{ }^{27} \mathrm{Al}$ has a nuclear quadrupole moment, with $\operatorname{spin} I=5 / 2$, which interacts with the electric field gradient caused by the nonspherically symmetric charge distribution around the ${ }^{27} \mathrm{Al}$ nucleus. Both line broadening/distortion and chemical shift changes may arise from those quadrupolar interactions resulting in more difficult interpretations of the ${ }^{27} \mathrm{Al}$ NMR spectra. Conversely, the high sensitivity of the ${ }^{27} \mathrm{Al}$ isotope, in $100 \%$ abundance with generally short relaxation times, allows the determination of very small amounts of aluminum in the samples. Spinlattice relaxation times $\left(\mathrm{T}_{1}\right)$ of ${ }^{27} \mathrm{Al}$ in zeolitic frameworks have been measured by Haase et $a l .{ }^{13}$ in the range of 0.3 to $70 \mathrm{~ms}$. Obeying Loewenstein's rule, where Al-O-Al linkages are forbidden, zeolites give rise to quite simple ${ }^{27} \mathrm{Al} \mathrm{NMR} \mathrm{spectra,} \mathrm{consisting} \mathrm{of} \mathrm{signals} \mathrm{due} \mathrm{only}$ to one type of tetrahedral aluminum environment, $\mathrm{Al}(\mathrm{OSi})_{4}$, in addition to the eventual octahedrally coordinated aluminum. The quantitative use of ${ }^{27} \mathrm{Al}$ MAS NMR is a valuable 
tool in probing the coordination, location and quantity of $\mathrm{Al}$ in zeolites, but less useful than ${ }^{29} \mathrm{Si}$ MAS NMR for the detailed study of lattice structure. ${ }^{14}$ 


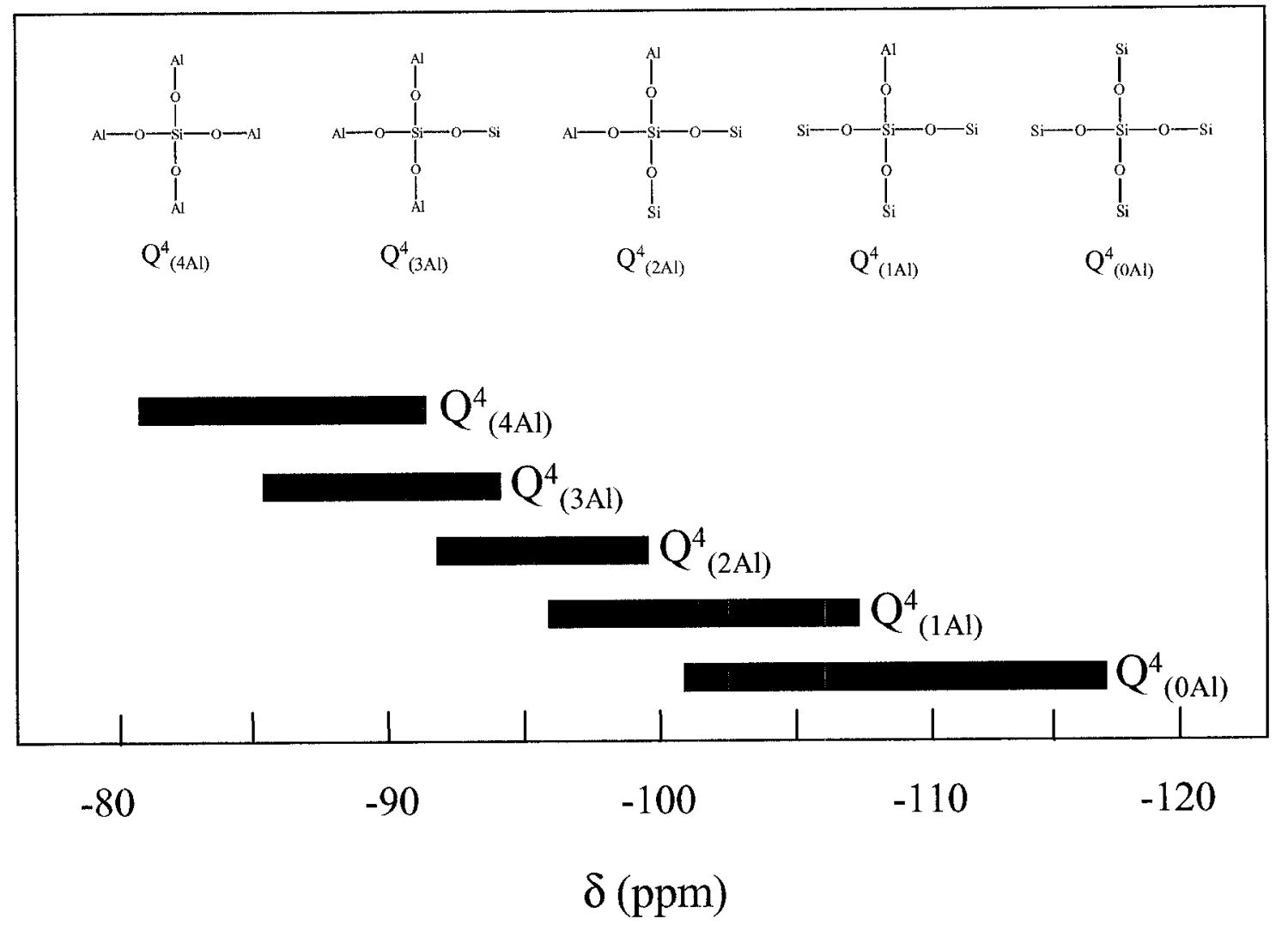

Fig 2.1 Ranges of ${ }^{29} \mathrm{Si}$ chemical shifts of $\mathrm{Q}^{4}(n \mathrm{Al})$ units in aluminosilicates. 


\section{Experimental}

\subsection{TGA experiments}

TGA measurements were performed using a 2050 Thermogravimetric Analyzer (TA Instruments) with nitrogen, helium or oxygen as the purge gas, where indicated, at a flow rate of $40 \mathrm{cc} / \mathrm{min}$. Where temperatures were to exceed $500^{\circ} \mathrm{C}$, open, platinum pans were utilized; otherwise, experiments were carried out in open, aluminum pans. Sample masses ranged between 5-20 mg. Before analysis, samples were dried to $300^{\circ} \mathrm{C}$, allowed to cool in a dessicator, then placed in an atmosphere of water $(97 \%$ humidity, maintained with a saturated $\mathrm{K}_{2} \mathrm{SO}_{4}$ solution), methanol or ethanol for several days. Samples were quickly placed into the TGA sample pan once removed from the solvent atmosphere.

\subsubsection{Sample weight loss measurements}

Experiments were run at a ramping rate of $5^{\circ} \mathrm{C} / \mathrm{min}$ to $300^{\circ} \mathrm{C}$, and subsequently kept isothermal for 60 minutes, with nitrogen flow. Weight loss experiments, where oxygen was utilized as the purge gas, were run at a ramping rate of $5^{\circ} \mathrm{C} / \mathrm{min}$ to $1000^{\circ} \mathrm{C}$, and subsequently kept isothermal for 60 minutes.

\subsubsection{Temperature cycle experiments}

Experiments were run with a temperature increase ramping rate of $5^{\circ} \mathrm{C} / \mathrm{min}$ to $350^{\circ} \mathrm{C}$, kept isothermal for 30 minutes, then a temperature decrease ramping rate of $2^{\circ} \mathrm{C} / \mathrm{min}$ to $25^{\circ} \mathrm{C}$, and also kept isothermal for 30 minutes. This cycle was repeated five times, with either nitrogen or helium as the purge gas. 


\subsection{Porosimetry measurements}

Nitrogen adsorption measurements at $77 \mathrm{~K}$ were carried out on samples NS1 and NS2 and were done using an ASAP Micrometrics 2010 porosimeter. Outgassing of the samples prior analysis was done for several days at $250^{\circ} \mathrm{C}$.

\subsection{PXRD measurements}

Powder diffraction patterns for NS1 and NS2 were collected on a Scintag X2 powder diffractometer $\left(\mathrm{CuK} \alpha 1=1.54056 \mathrm{~nm}, 5^{\circ} \leq 2 \theta \leq 65^{\circ}, 0.02^{\circ}\right.$ step interval, 0.5 second count time per data point). Powder pattern search and match was done through the JCPDS database.

\section{$2.4 \quad$ SS MAS NMR spectroscopy methods}

\subsection{1 ${ }^{13}$ C SS MAS NMR experiments}

${ }^{13} \mathrm{C}$ SS MAS NMR experiments were performed on a Tecmag Apollo $200 \mathrm{MHz}$ spectrometer using $7 \mathrm{~mm}$, double-bearing magic-angle spinning zirconia rotors. $\mathrm{All}^{13} \mathrm{C}$ spectra were spun at approximately $4000 \mathrm{~Hz}$. Experiments were performed at a resonance frequency of $50.33 \mathrm{MHz}$. Chemical shifts were referenced to adamantane. Proton decoupling was applied, and at least 4096 scans were acquired for each spectrum with sweep width of $20000 \mathrm{~Hz}$, a pulse length of $10 \mu \mathrm{s}$, and a repetition time of $10 \mathrm{~s}$. Analysis and curve fitting of spectra was done using Microcal Origin 6.0.

\subsection{2 ${ }^{29}$ Si SS MAS NMR experiments}

${ }^{29} \mathrm{Si}$ SS MAS NMR experiments were performed on a Tecmag Apollo $200 \mathrm{MHz}$ 
spectrometer using $7 \mathrm{~mm}$, double-bearing magic-angle spinning zirconia rotors. $\mathrm{All}{ }^{29} \mathrm{Si}$ spectra were spun at approximately $4000 \mathrm{~Hz}$. Experiments were performed at a resonance frequency of $39.76 \mathrm{MHz}$. Chemical shifts were referenced to tetramethylsilane. Proton decoupling was applied, and at least 1024 scans were acquired for each spectrum with sweep width of $10000 \mathrm{~Hz}$, a pulse length of $11 \mu \mathrm{s}$, and a repetition time of 100s. Analysis and curve fitting of spectra was done using Microcal Origin 6.0.

\subsection{3 ${ }^{27}$ Al SS MAS NMR experiments}

${ }^{27} \mathrm{Al}$ SS MAS NMR experiments were performed on a Tecmag Apollo $200 \mathrm{MHz}$ spectrometer using $7 \mathrm{~mm}$, double-bearing magic-angle spinning zirconia rotors. $\mathrm{All}{ }^{27} \mathrm{Al}$ spectra were spun at approximately $4000 \mathrm{~Hz}$. Experiments were performed at a resonance frequency of $52.15 \mathrm{MHz}$. Chemical shifts were referenced to a dilute aqueous solution of $\mathrm{Al}(\mathrm{NO})_{3}$. Proton decoupling was applied, and at least 2048 scans were acquired for each spectrum with sweep width of $20000 \mathrm{~Hz}$, a pulse length of $2 \mu \mathrm{s}$, and a repetition time of $0.2 \mathrm{~s}$. Analysis and curve fitting of spectra was done using Microcal Origin 6.0.

\subsection{General procedure for the coating of zeolites}

Approximately 2-3g of zeolite ${ }^{15}$ was placed in a 2-necked round bottomed flask. The zeolite was heated to $150-200^{\circ} \mathrm{C}$ under vacuum on a Schlenk line for 2 days to ensure complete removal of water from the zeolite structure. After heating, the flask with the contents was allowed to cool. A solution of the coating agent was prepared by diluting the organosilane: SS (Pierce), TMCS (Aldrich), or Pyrene-Cl in $n$-pentane (Fisher Scientific) 
$(1: 10 \mathrm{~m} / \mathrm{v})$. Maintaining vacuum in the flask, and using a syringe, the coating solution was introduced to the zeolite, enough to wet it completely. The system was then purged with $\mathrm{N}_{2}$ gas, and opened to atmosphere. To the flask was added excess of the same solution $(50 \mathrm{~mL})$. Stirring was then initiated and maintained overnight. After stirring was stopped, the excess solution was decanted, and the zeolite was then rinsed with pure $n$-pentane (x3). After rinsing, the supernatant liquid was decanted again. The remaining zeolite was dried under vacuum to remove excess solvent. The coated zeolite was then stored in a vial, ready for analysis.

\subsubsection{Pre-coating of Glass Vessels}

This procedure is followed if a silation reaction is to be done. Because the surface of glass possesses hydroxyl groups, it is necessary to protect the vessel from reacting with the silating reagent needed.

A vessel, dried in an oven at $150^{\circ} \mathrm{C}$, and cooled to room temperature, was filled with a solution containing a 1:10 (m/v) ratio of organosilane (SS, TMCS, etc.) and $n$-pentane. This solution was allowed to stand in the vessel for at least 30 minutes. After the time had elapsed, the contents of the vessel were decanted. The vessel was then inverted and allowed to air dry for at least one half hour. The vessel was then rinsed three times with pure $n$ pentane and air-dried.

\subsection{General Pyrene-derivative Synthetic Procedure}

All reagents were obtained from Aldrich and used as received unless otherwise 
indicated. All solvents were used directly from the bottle. Glassware and magnetic stir bars were dried in the oven at $150^{\circ} \mathrm{C}$ prior to use.

\subsubsection{Synthesis of Pyrene-TBDMS}

To a 2-necked, $500 \mathrm{~mL}$ round-bottomed (r.b.) flask equipped with a magnetic stir bar and a reflux condenser, was added $1.5 \mathrm{~g}(0.0052 \mathrm{~mol})$ of PBA (1) (purified by sublimation) and a molar equivalent of TBDMS. The reaction was conducted in ethyl acetate (EtOAc, Fisher) ( $\sim 250 \mathrm{~mL})$, under reflux overnight, resulting in a clear, yellow supernatant liquid with a light brown precipitate.

The resulting product was then extracted with EtOAc. After drying over $\mathrm{MgSO}_{4}$ (Fisher), the solution was filtered and evaporated under vacuum to produce a yellow, pungent-smelling solid. The yield was $60 \%$. The product, Pyrene-TBDMS (2), was dried under a stream of $\mathrm{N}_{2}$ gas, and was purified by sublimation to give a pale, yellow powder. ' $\mathrm{H}$

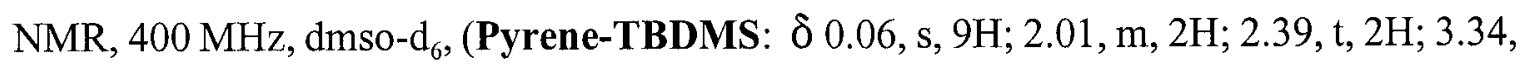
s, $6 \mathrm{H} ; 7.93-8.41$, aromatic, $9 \mathrm{H}),($ EtOAc: $\delta 1.2, \mathrm{t}, 3 \mathrm{H} ; 2.01, \mathrm{~s}, 3 \mathrm{H} ;$; 4.06, q, 2H), (dmso: 2.50, p, 6H), m.p. $184-186^{\circ} \mathrm{C}$.

\subsubsection{Synthesis of Red-Pyrene-TBDMS}

To a 2-necked, $250 \mathrm{~mL}$ r.b. flask equipped with a magnetic stir bar and a reflux condenser, was added $1 \mathrm{~g}(0.0025 \mathrm{~mol})$ of Pyrene-TBDMS (2) and two molar equivalents of $\mathrm{NaBH}_{4}$. The reaction was conducted in EtOAc $(\sim 250 \mathrm{~mL})$, under reflux overnight, yielding a yellow precipitate. The resulting product was then extracted with EtOAc. After 
drying over $\mathrm{MgSO}_{4}$, the solution was filtered and evaporated under vacuum to produce a yellow solid. The yield was $80 \%$. The product, Red-Pyrene-TBDMS (3), was dried under a stream of $\mathrm{N}_{2}$ gas, and purified by sublimation to give a yellow powder. ${ }^{\prime} \mathrm{H} N M R, 400$ MHz, dmso-d ${ }_{6}$, Red-Pyrene-TBDMS: $\delta_{\delta} 0.48, \mathrm{~d}, 6 \mathrm{H} ; 2.88, \mathrm{t}, 2 \mathrm{H} ; 2.99, \mathrm{~s}, 2 \mathrm{H} ; 3.84, \mathrm{t}, 4 \mathrm{H}$; 7.94-8.49, aromatic, 9H), (EtOAc: $\delta 1.33, \mathrm{~s}, 3 \mathrm{H} ; 1.66, \mathrm{t}, 3 \mathrm{H}, ; 4.52, \mathrm{q}, 2 \mathrm{H}),(\mathrm{dmso}: 2.50, \mathrm{p}$, $6 \mathrm{H})$, m.p. $179-183^{\circ} \mathrm{C}$.

\subsubsection{Synthesis of Pyrene-But-OH}

To a 2-necked, $250 \mathrm{~mL}$ r.b. flask equipped with a magnetic stir bar and a reflux condenser, was added 0.52g (0.0013 mol) of Red-Pyrene-TBDMS (3) and four molar equivalents of TBAF. The reaction was conducted in EtOAc $(\sim 250 \mathrm{~mL})$, under reflux overnight, resulting in a clear, yellow liquid.

The resulting product was then extracted with EtOAc. After drying over $\mathrm{MgSO}_{4}$, the solution was filtered and evaporated under vacuum to produce a yellow solid, with $75 \%$ yield. The product, Pyrene-But-OH (4), was dried under a stream of $\mathrm{N}_{2}$ gas, and purified by sublimation to give a pale-yellow powder. ${ }^{1} \mathrm{H}$ NMR, $400 \mathrm{MHz}$, dmso-d ${ }_{6}$, (Pyrene-ButOH: $\delta 0.85, \mathrm{t}, 1 \mathrm{H} ; 0.90, \mathrm{t}, 0.5 \mathrm{H} ; 2.01, \mathrm{p}, 2 \mathrm{H} ; 2.39, \mathrm{t}, 4 \mathrm{H} ; 3.34, \mathrm{~m}, 4 \mathrm{H} ; 7.93-8.41$, aromatic, 9H; 12.12, bs, $1 \mathrm{H})$, (EtOAc: $\delta 1.33, \mathrm{t}, 3 \mathrm{H} ; 2.01, \mathrm{~s}, 3 \mathrm{H}, ; 4.06, \mathrm{q}, 2 \mathrm{H}),(\mathrm{dmso}: 2.50, \mathrm{p}, 6 \mathrm{H})$, m.p. $167-175^{\circ} \mathrm{C}$.

\subsubsection{Synthesis of Pyrene-Cl}

To a pre-coated, 2-necked, $500 \mathrm{~mL}$ r.b. flask, closed to atmosphere, equipped with a 
magnetic stir bar and flushed with nitrogen, was added $0.52 \mathrm{~g}(0.0013 \mathrm{~mol})$ of Pyrene-ButOH (4) and slight molar excess of DCDMS $(0.2 \mathrm{~mL})$. The reaction was conducted under nitrogen, in anhydrous EtOAc $(\sim 250 \mathrm{~mL})$, resulting in a clear, yellow liquid.

The solvent was isolated by evaporating the solvent under a stream of nitrogen gas to produce a yellow-brown solid, with moderate yield. The product, Pyrene-Cl (5) was dried further under a stream of $\mathrm{N}_{2}$ gas, and stored in a drybox, away from moisture. ${ }^{1} \mathrm{H} N \mathrm{NR}, 400$ MHz, dmso-d ${ }_{6}$, (Pyrene-Cl: $\delta-0.11$ to -0.02 , bs, d, 6H; 1.2, t, 2H, 2.02, m, 2H; 2.39, t, 2H; 3.34, t, 2H; 7.63-8.41, aromatic, 9H), (DCDMS: $\delta 0-0.13, \mathrm{~d}, \mathrm{t}, \mathrm{s}, 6 \mathrm{H}),($ EtOAc: $\delta 1.33, \mathrm{t}, 3 \mathrm{H}$; $2.01, \mathrm{~s}, 3 \mathrm{H}, ; 4.03, \mathrm{q}, 2 \mathrm{H})$, (dmso: $2.50, \mathrm{p}, 6 \mathrm{H})$, m.p. $181-183^{\circ} \mathrm{C}$.

\subsection{Adsorption experiment setup}

All zeolite samples were dried in an oven at $150^{\circ} \mathrm{C}$ for several days, then heated at $300^{\circ} \mathrm{C}$ for 24 hours, then placed in a dessicator to cool before use. Duplicates of each zeolite sample were weighed out $(0.06-0.08 \mathrm{~g})$ and were then placed in pre-weighed glass weighing bottles. The samples were then placed in a closed environment of either saturated water (humidity kept at $97 \%$ with a saturated $\mathrm{K}_{2} \mathrm{SO}_{4}-\mathrm{H}_{2} \mathrm{O}$ solution), methanol or ethanol, and allowed to equilibrate over several days, in a cool, dark place. Weight readings were taken at prescribed intervals, taking care to place the lids on the vials as quickly as possible once the storage container was opened. After observing the mass of the samples equilibrate after 15 hours, the samples were placed again in the oven for several days to drive off all solvent, allowed to cool in a dessicator and re-weighed. 


\section{References}

1. TA Intruments: Thermal Analysis \& Rheology. A Subsidiary of Waters Corporation. 1997, New Castle, DE: TA Instruments, Inc.

2. Rouquerol, F., J. Rouquerol, and K.Sing, Adsorption by Powders \& Porous Solids: Principles, Methodology and Applications. 1999, London: Academic Press. 467.

3. West, A.R., Solid State Chemistry and its Applications. 1984: John Wiley \& Sons Ltd.

4. Atkins, P., Physical Chemistry. 5th ed. 1994, New York: W. H. Freeman and Company.

5. Sarv, P., High Resolution Solid-State NMR Studies of Zeolites. 1998, Tartu: Tartu University Press.

6. Andrew, E.R., A. Bradbury, and R.G. Eades, Nature, 182, 1958, 1659.

7. Fitzgerald, J.J. and S.M. DePaul, Solid-State NMR Spectroscopy of Inorganic Materials: An Overview, in Solid-state NMR spectroscopy of inorganic materials, J.J. Fitzgerald, Editor. 1999, American Chemical Society: Washington, DC. p. 2-133.

8. Stöcker, M., Review on recent NMR results, in Advanced Zeolite Science and Applications: Studies in Surface Science and Catalysis, J.C. Jansen, M. Stocker, H.G. Karge, and J. Weitkamp, Editors. Vol. 85. 1994, Elsevier Science.

9. Lippmaa, E., M. Maegi, A. Samoson, G. Engelhardt, and A.R. Grimmer, J. Am. Chem. Soc., 102, 1980, 4889.

10. Lippmaa, E., M. Maegi, A. Samoson, M. Tarmak, and G. Engelhardt, J. Am. Chem. Soc., 103, 1981, 4992.

11. Engelhardt, G., Trends in Anal. Chem., 8, 1989, 343.

12. Engelhardt, G., Stud. Surf. Sci. Catal., 58, 1991, 285.

13. Haase, J., H. Pfeifer, W. Oehme, and J. Klinowski, Chem. Phys. Lett., 150, 1988, 189. 
14. Engelhardt, G. and D. Michel, High-Resolution Solid-State NMR of Silicates and Zeolites. 1987, Norwich: John Wiley \& Sons Ltd.

15. The zeolites being used were: Linde $4 \AA$, Linde $5 \AA$ : synthesized, Union Carbide Corp., natural Stilbite and Mordenite. The natural samples were ground with a mortar and pestle before coating was done. 


\section{Chapter III}

\section{Modification of Surfaces and the Synthesis of a Novel Coating Material}

1. Modification of surfaces $\ldots \ldots \ldots \ldots \ldots \ldots \ldots \ldots \ldots \ldots \ldots \ldots \ldots$

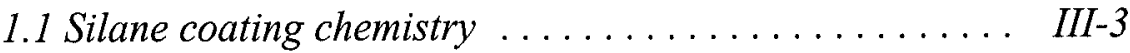

1.2 Coating of glass surfaces $\ldots \ldots \ldots \ldots \ldots \ldots \ldots$ III-5

1.3 Modification of zeolites and zeolite-type materials . . . III-6

2. Synthesis of a new coating material: Pyrene-Cl . . . . . . III-6

2.1 Synthetic strategy $\ldots \ldots \ldots \ldots \ldots \ldots \ldots \ldots \ldots$ III-7

3. Synthesis of a novel pyrene coating: results $\ldots \ldots \ldots \ldots \ldots$ III-11

References ........................... III 15 


\section{Modification of Surfaces}

Material science is typically driven by a desire to produce novel compounds or to modify known compounds for various applications. In this study, modification of external zeolite surfaces is examined and the effects of such modification is determined. Surface modifications can be carried out most conveniently with the use of organosilanes. A hydrolyzable chlorine substituent allows covalent grafting of hydrophobic organic functional groups to otherwise hydrophilic zeolites.

The strategy of adding a coating to zeolite structures is currently the subject of intensive study due to the potential applications such as protective coatings, optoelectronic devices, catalysts and modified electrodes, as well as for fundamental studies of wetting, adhesion, and general behaviour. ${ }^{1}$ In particular, organosilanes have rapidly gained popularity, and are widely used. For instance, alkylsilane films have been one of the most widely used classes of coatings due to the fact that they can be deposited on a variety of substrates and are both thermally and chemically stable. ${ }^{2}$

Arkles $^{3}$ describes in his review on tailoring surfaces with silanes, that encounters between dissimilar materials involve at least one member that is siliceous, or that has surface chemistry with siliceous properties such as silicates, aluminates, borates and so on. Interfaces involving such materials have become a dynamic area of chemistry where surfaces have been modified in order to generate desired heterogeneous environments or to incorporate the bulk properties of different phases into a uniform composite structure. In 
general, a coating and substrate conglomerate is produced when a material is exposed to a modifying agent. The modifying agent of interest is that of an organosilane, with the general formula $\mathrm{R}_{\mathrm{n}} \mathrm{SiX}_{(4-\mathrm{n})}$ where $\mathrm{X}$ is a hydrolyzable group, usually an alkoxy, acyloxy, amine, or as in this study, a halogen. The $\mathrm{R}$ group is a non-hydrolyzable organic group in possession of a functionality imparting desired characteristics that can be rationally designed.

\subsection{Silane coating chemistry}

Silane coatings are chosen for reactivity and functionality that is compatible with the substrate to be coated. This means that the hydrolyzable $(\mathrm{X})$ group has predictable chemical behaviour, and the nonhydrolyzable $(\mathrm{R})$ group has the desired characteristics that will give the coating layer the properties being sought. The performance of a coating agent is independent of the nature of the $\mathrm{X}$ groups if adequate moisture is available for hydrolysis, either from the solvent in which the reaction is taking place, or at the site of reaction. ${ }^{4}$

Additionally, either extremity of the silane molecule can undergo chemical modification, either separately, or simultaneously. The X groups can be exchanged or hydrolyzed without altering the $\mathrm{R}$ group. Chemical modification of the $\mathrm{R}$ group may precede application to a surface or may take place at a surface after silylation. ${ }^{4}$ In particular, this overall chemistry of coating surfaces is known to work, because the $\mathrm{X}$ group reacts readily with hydroxyl groups on substrate surfaces, specifically in the case in modifying the surfaces of zeolites (Fig. 3.1). ${ }^{5}$ 


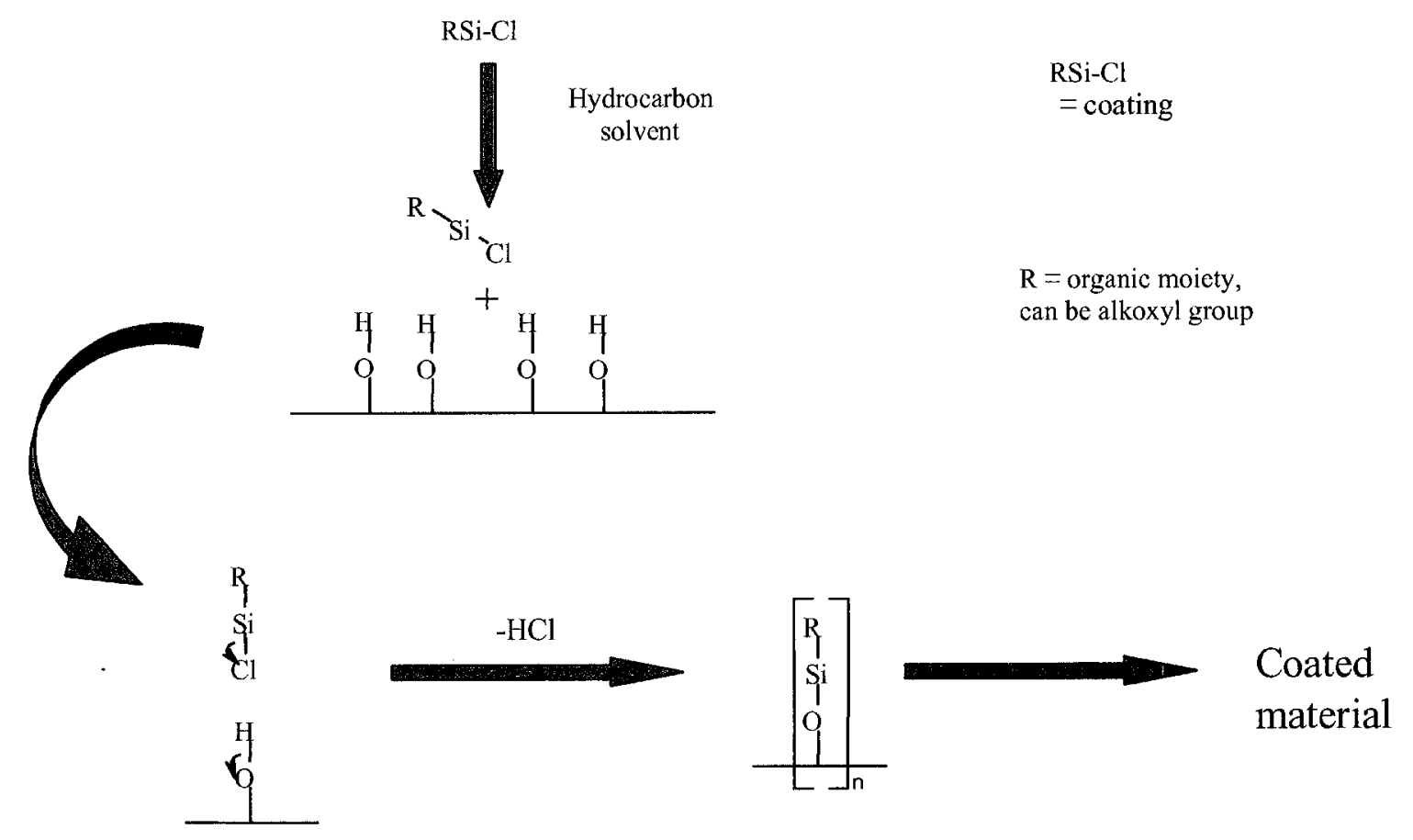

Fig. 3.1 A schematic representation of the reaction of a coating material with a substrate. 


\subsection{The coating of glass surfaces}

The surface treatment of materials, such as metal oxide surfaces, clay minerals, glass and zeolites with organochlorosilanes has widespread use in diverse areas such as the production of self-assembled monolayers, chromatography and xerography. ${ }^{6}$ The successful application of the treatment of materials with organochlorosilanes has been limited by the variability in the quality of the films produced. Much of the day-to-day variance can be traced to a critical dependence on reaction temperature and on the level of surface water. ${ }^{6}$ Most silation reactions are performed near room temperature in non-aqueous solutions. ${ }^{6}$ Under these conditions, surface hydroxyls are needed to hydrolyze the chlorosilane moiety, to form an intermediate silanol species, which reacts with surface hydroxyl groups to form the final chemical species.

It is well known that chlorosilanes react readily with all hydroxyl groups to generate hydrochloric acid. ${ }^{7}$ Utilizing this reaction, many different organic functional groups can be introduced onto glass surfaces through covalent bonds. ${ }^{7}$ Surface modification specifically of porous glass membranes by a covalently bonded molecular monolayer is thus a special way of altering membrane performance to introduce various organic functionalities. ${ }^{7}$ The voids within porous glass are rigid in shape and are modified by the organic monolayer. These surface-modified membranes can then exhibit high thermal stabilities and high permeance as well as organic functionality, or high selectivity to organic compounds that come into contact with the modified glass. 


\subsection{Modification of zeolite and zeolite-type materials}

Many studies have investigated methods to control the surface properties of zeolites and related materials by exposing them to silicon compounds..$^{5,810}$ It is the presence of the hydroxyl groups on the surface that can be used to anchor -SiR groups via a silanation process, which can be tailored to function as pore-blockers, or selectively change the surface properties and adsorption capacity. The modification can be carried out using organosilicon compounds containing one or several functional groups, which makes further reactions on the surface of the modified adsorbents possible; in this way, selective adsorbents can be prepared. ${ }^{11}$ It has been observed that modifying zeolites and related materials causes a change in adsorption properties, where the adsorption capacity of such adsorbents is higher than that of the chemically unmodified ones. ${ }^{11}$ It is the modification of zeolite surfaces that is of interest in this study, specifically with the coating materials: SurfaSil ${ }^{\mathrm{TM}}$ (SS), trimethylchlorosilane (TMCS) and newly synthesized pyrene-containing molecule, pyrene-1(4-butyl-dimethylchlorosilyl) ether (Pyrene-Cl) (Fig. 3.2).

\section{Synthesis towards a new coating material: Pyrene-Cl}

Commercially available, straight-chained organosilane molecules have been the most popular type of coating material in the surface modification of substrate surfaces. Some slightly branched materials have also been utilized, but what has not been extensively studied in the literature is the use of larger organic functional groups, such as pyrene, that are part of the organosilane coating used to coat a surface. This is what is intended in this study: the 
synthesis of a new coating material and the introduction via a covalent bond to the surfaces of zeolite LTA of the $4 \AA$ and $5 \AA$ types.

\subsection{Synthetic Strategy}

Pyrene butyric acid was the starting point of the synthetic sequence as shown in Fig. 3.3. The carboxylic acid functionality is the reactive group used in the modification of this compound, that will be useful for the modification of a zeolite surface. Specifically, it is the protection of the carboxyl -OH group that will first be accomplished, the carbonyl will be removed by a double reduction reaction, followed by the removal of the protecting group to yield a terminal alcohol that will then be available for the attachment of a chloro-silyl group.

The strategy of protection of a hydroxyl functionality and its subsequent deprotection at a later stage is frequently used in multi-step synthesis. ${ }^{12,13}$ Of all the hydroxyl protecting groups, $t$-butyldimethylchlorosilyl (TBDMS) ether has become the most popular and widely used means of protecting groups because of its ease of preparation, and its stability to a wide range of reaction conditions. ${ }^{14}$ The reduction of the carbonyl group, that results from the attachment of the TBDMS protecting group, is performed using two molar equivalents of $\mathrm{NaBH}_{4}$, which works well in reducing carbonyls in good yield. ${ }^{15}$ Numerous methods are now available in the literature ${ }^{14}$ for the deprotection of TBDMS ethers under a variety of conditions. The transformation of an alcohol to its corresponding TBDMS ether has been a common and useful method for protecting the hydroxyl group since its introduction by Corey ${ }^{16}$ in 1972. 

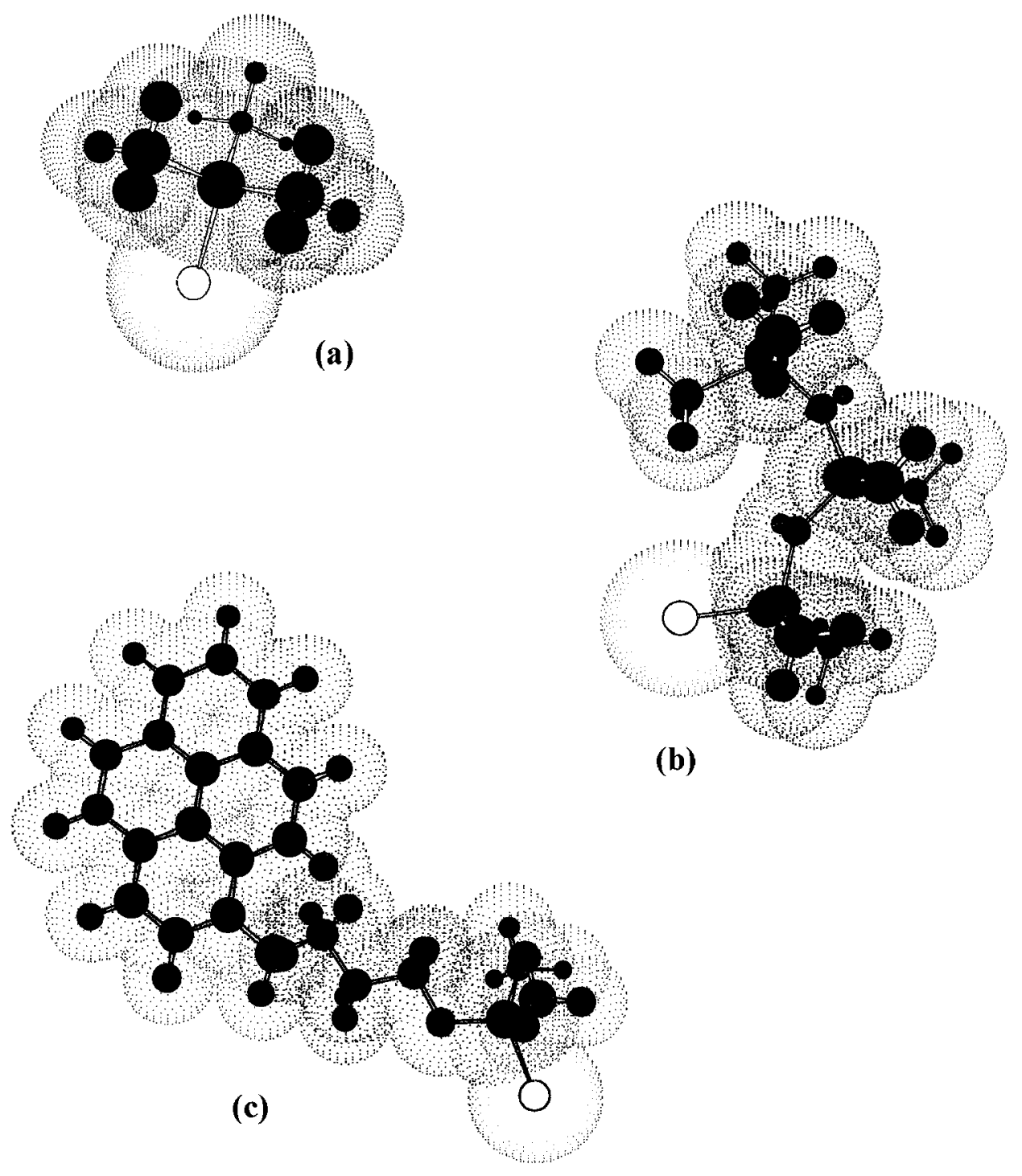

(b)

Fig. 3.2 Ball-and-stick model, with van der Waal's radii, representation of the organosilane coating materials: a, trimethylchlorosilane; $b$, SurfaSil $^{\mathrm{TM}}$; c, pyrene-1-(4-butyl-dimethylchlorosilyl) ether. 


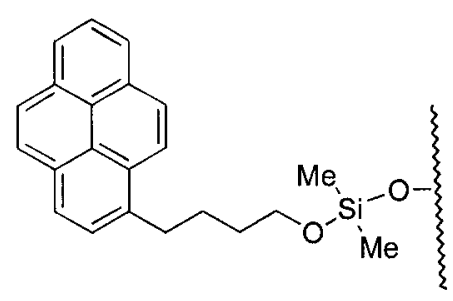<smiles>C[Si](Cl)(Cl)OCCCCc1ccc2ccc3cccc4ccc1c2c34</smiles><smiles>C[Si](C)(Cl)Cl</smiles><smiles>OCCCCc1ccc2ccc3cccc4ccc1c2c34</smiles>
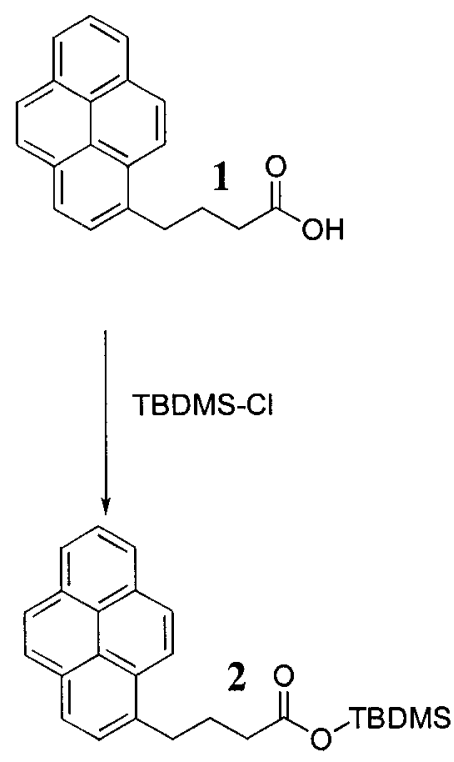

$\mathrm{NaBH} 4$
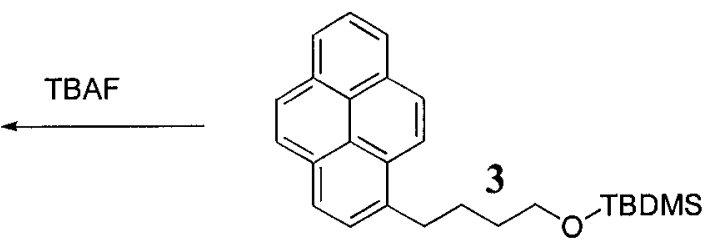

Fig. 3.3 Sequence of steps for the synthesis of pyrene-1-(4-butyldimethylchlorosilyl) ether; the last step shows the introduction of the material to a zeolite surface. 
One of the most effective ways for the cleavage of silyl ethers is based on the exploitation of the high affinity of silicon towards fluoride ions. ${ }^{14}$ Thus, a number of reagents involving one form of the fluoride ion or another, such as tetrabutylammonium fluoride (TBAF), the reagent of choice in this study, boron trifluoride etherate, hydrofluoric acid, have been developed for the deprotection of TBDMS ethers. Among these, TBAF is the most frequently used. Concerns have arisen to the use of TBAF as a deprotection reagent due to the basicity of the bare fluoride ion in tetrahydrofuran, which directly affects cleavage of TBDMS ethers of the $\beta$-ketol system of phenolic compounds and may promote $\beta$ elimination reactions, transacylations and other base-catalyzed undesired side reactions. However, TBAF is used only in one aspect of the workup in the reaction sequence described in this study; these difficulties are not expected. Only one hydroxyl group is to be protected, and any complications that have been described ${ }^{14,17}$, involve either several protected sites, or complex systems. Once the protecting group is removed, the resulting terminal hydroxyl functionality is then available for the covalent linkage of an organochlorosilane, by exploiting the same chemistry that has been used in the coating of glass surfaces and other materials that possess surface hydroxyl groups. In this case, a dichloro-organosilane where one chlorine is to react with the hydroxyl of this pyrene-containing organic coating material, and the remaining $\mathrm{Cl}$ is available for reaction with surface hydroxyls of the substrate to be coated, is introduced. 


\section{Synthesis of a novel pyrene coating}

The modification and substitution of sites of pyrene have been studied previously ${ }^{18}$; however, the use of a pyrene-substituted molecule as a surface modification agent is not understood. The chemistry at each individual synthetic step has been well documented; hence, the surface chemistry of the pyrene-substituted coating agent should also be predictable. Observation of the changes to the molecule were monitored via ${ }^{1} \mathrm{H}$ NMR spectroscopy. Evidently, the aromatic moiety, in all steps, remained unmodified; therefore, what was being searched for was evidence of the appearance of proton signals of the groups being added, namely the TBDMS protecting group after the first synthetic step. Using the ${ }^{1} \mathrm{H}$ NMR spectrum of PBA, which was purified via sublimation, as a reference, the subsequent spectra from each following steps were compared. There was an appearance of several new peaks after the introduction of TBDMS resulting in the formation of 2 . Methyl protons in the 0-1 ppm range are observed. After the double reduction step in the synthesis of 3, it would be expected to see the appearance of more alkyl proton resonances, as was indeed the case, at $1.25 \mathrm{ppm}$. In the next step of the sequence, in producing 4 , the ${ }^{1} \mathrm{H} N M R$ spectrum revealed the appearance of a new peak at $\delta 0.83$, indicating that there was the production of a terminal hydroxyl, and the absence of the methyl and $t$-butyl signals from TBDMS confirmed the success of this step. The final step, where the introduction of DCDMS to 4 to produce 5 (Fig 3.4), methyl proton signals appeared, from lowfield to highfield, as a cluster of a singlet, triplet, doublet, doublet and singlet in the region around 
$0 \mathrm{ppm}$. The last two peaks at -0.08 and $-0.04 \mathrm{ppm}$ give an integral of $6 \mathrm{H}$. This appearance confirms the successful addition of DCDMS to 4. The remainder are attributed to DCDMS that was not successfully removed from the product. The other sets of peaks in the spectrum are assigned the following: the pyrene- $\mathrm{H}$ resonances for nine hydrogens appear in the aromatic region of 7.94-8.42 ppm; hydrogen resonances for the alkyl chain appear: a triplet at $3.34(2 \mathrm{H})$, a triplet at $2.34(2 \mathrm{H})$ and a pentuplet at $2.01 \mathrm{ppm}(2 \mathrm{H})$, and a triplet at $1.21 \mathrm{ppm}$ (2H). A peak at $4 \mathrm{ppm}$ is attributed to traces of the reaction solvent, EtOAc, that had not been removed. All ${ }^{1} \mathrm{H}$ NMR spectra for the preliminary steps of the reaction sequence products are given in the Appendix. Figure 3.5, displaying the mass spectrum (ES+), does not seem to show the $\mathrm{M}^{+1}$ peak for the compound proper; however, the larger peaks that do show prominence are 242.3 and 186.3 , which correspond to the Pyrene-1-butyl moiety and the O-DCDMS moiety, respectively. Evidently, it is the C-O bond connecting these two moieties that is one of the weakest points in the molecule. 
Chapter III Modification of Surfaces and the Synthesis of a Novel Coating Material

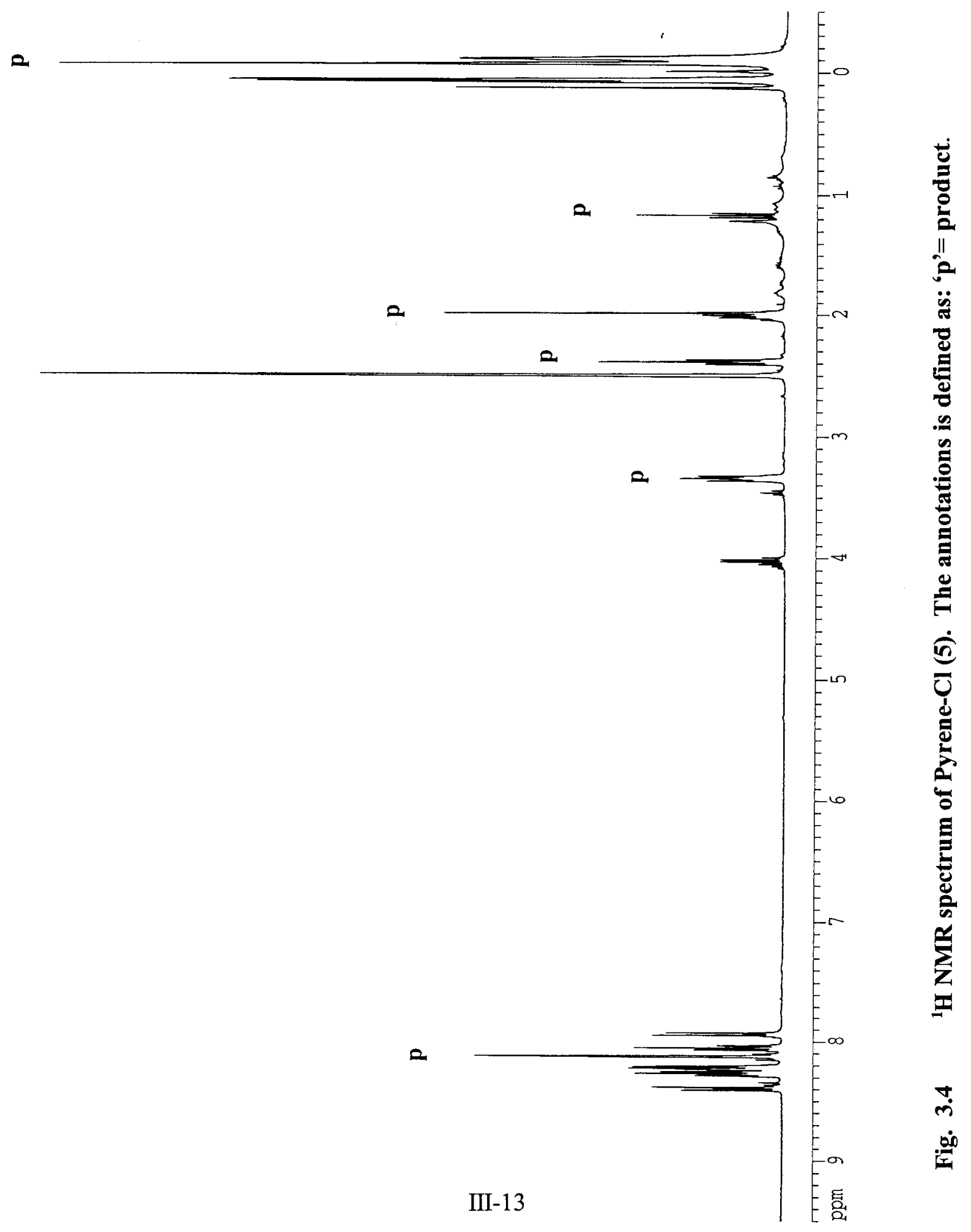




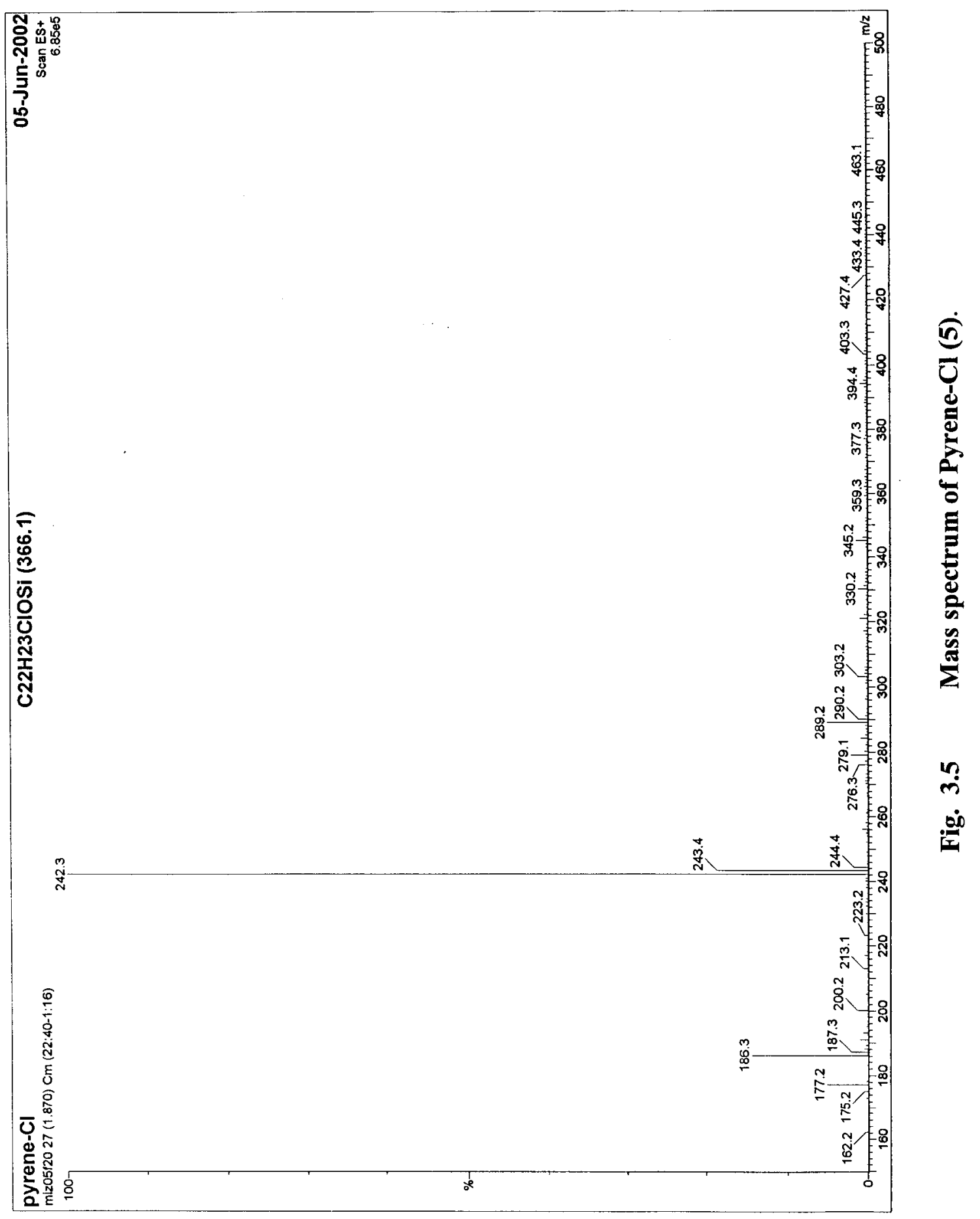

III-14 


\section{References}

1. Gao, W. and L. Reven, Langmuir, 11, 1995, 1860.

2. Gao, W., L. Dickinson, C. Grozinger, F.G. Morin, and L. Reven, Langmuir, 13, $1997,115$.

3. Arkles, B., Chemtech, 7, 1977, 766.

4. Plueddemann, E.P., Chemistry of Silane Coupling Agents, in Midland Macromolecular Monographs: Silylated Surfaces, D.E. Leyden and W.T. Collins, Editors. 1978, Gordon and Breach Science Publishers: New York.

5. Kim, J.-H., Y. Ikoma, and M. Niwa, Microporous and Mesoporous Materials, 32, $1999,37$.

6. Tripp, C.P., R.P.N. Veregin, M.N.V. McDougall, and D. Osmond, The Importance of a Surface $\mathrm{Si}_{\mathrm{s}}-\mathrm{O}$-Si Bond between Alkylchlorosilanes and Silica in Defining the Distribution of Water on the Surface, in Chemically Modified Surfaces: Recent Developments, J.J. Pesek, M.T. Matuska, and R.R. Abuelafiya, Editors. 1995, Royal Society of Chemistry: Cambridge. p. 89.

7. Kuraoka, K., Y. Chujo, and T. Yazawa, Journal of Membrane Science, 182, 2001, 139.

8. Hu, Z., W. Lihui, J. Dong, Y. Wang, S. Chen, and S. Peng, Microporous and Mesoporous Materials, 28, 1999, 49.

9. Zhao, X.S., G.Q. Lu, and X. Hu, Microporous and Mesoporous Materials, 41, 2000, 37.

10. Jaroniec, C.P., M. Kruk, and M. Jaroniec, J. Phys. Chem. B, 102, 1998, 5503.

11. Leboda, R. and A. Dabrowski, Complex carbon-mineral adsorbents: preparation, surface properties and their modification., in Adsorption on New and Modified Inorganic Sorbents, A. Dabrowski and V.A. Tertykh, Editors. Vol. 99. 1996, Elsevier Science B. V.: Amsterdam. p. 115-189.

12. Nelson, T.D. and R.D. Crouch, Synthesis - Journal of Synthetic Organic Chemistry, (9), 1996, 1031. 
13. Crouch, R.D., M. Stieff, J.L. Frie, A.B. Cadwallader, and D.C. Bevis, Tetrahedron Letters, 40, 1999, 3133.

14. Ranu, B.C., U. Jana, and A. Majee, Tetrahedron Letters, 40, 1999, 1985.

15. March, J., Advanced Organic Chemistry: Reactions, Mechanisms, and Structure. 4th ed. 1992, New York: John Wiley \& Sons, Inc.

16. Corey, E.J. and A. Venkateswarlu, J. Am. Chem. Soc., 94, 1972, 6190.

17. Bajwa, J.S., J. Vivelo, J. Slade, O. Repić, and T. Blacklock, Tetrahedron Letters, 41, 2000, 6021 .

18. Vollmann, H., H. Becker, M. Corell, and H. Streeck, Justus Liebigs Annalen Der Chemie, 531, 1937, 1-159. 


\section{Chapter IV}

\section{Results and Discussion}

1. Thermal Gravimetric Analysis (TGA)

$I V-2$

1.1 Initial survey of weight loss of coated vs non-coated zeolites .................... IV-2

1.2 Cycle experiments $\ldots \ldots \ldots \ldots \ldots \ldots \ldots \ldots \ldots$

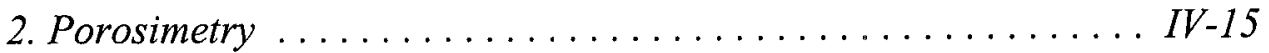

3. Powder X-Ray diffraction (PXRD) of the natural zeolite samples IV-17

4. Solid State Nuclear Magnetic Resonance (NMR) Spectroscopy . . IV-21

$4.1{ }^{13} \mathrm{C}$ SS NMR spectroscopy of coated LTA $4 \AA \ldots \ldots \ldots I V-21$

$4.2{ }^{29} \mathrm{Si}$ SS NMR spectroscopy of the natural zeolite samples $I V-21$

$4.3^{27} \mathrm{Al}$ SS NMR spectroscopy of the natural zeolite samples. IV-28

5. Uptake experiments ..................IV-28

References ........................ IV-34 


\section{Chapter IV Results and Discussion}

\section{Thermal Gravimetric Analysis (TGA)}

All samples were held in a closed atmosphere for at least several days to allow for equilibration of the atmosphere within the container, and for optimum uptake of solvent by the zeolite samples. The samples were placed out of direct sunlight to ensure ambient temperature conditions. After the waiting period, TGA experiments were carried out to determine the maximum loss of solvent. In the case of the temperature cycle experiments, gains and losses in sample mass were observed in a repeated manner. Those TGA plots not shown in this chapter and are presented in the Appendix. It should be noted that to determine whether the materials were going to undergo solvent uptake in the solvent atmosphere, preliminary experiments were conducted in order to determine whether any observable effect could be noted. Hence, TGA experiments were preformed to provide some background as to the behaviour of the zeolite exposed to various solvent atmospheres.

\subsection{Initial survey of weight loss of coated vs. non-coated zeolites}

In general, upon heating the samples to $1000^{\circ} \mathrm{C}$ under oxygen flow to determine total weight loss of both neat and coated zeolite samples, it can be stated that no pattern can be discerned as to the overall weight loss of the coated samples versus the neat samples. However, this does not indicate that no weight loss due to the burning off of the coating material occurred. This loss of coating material is observed in the temperature regions of $250-550^{\circ} \mathrm{C}$, as shown in the TGA plots. Table 4.1 summarizes the total weight loss of all zeolite samples to $1000^{\circ} \mathrm{C}$. 
Table 4.1 Total weight loss as a \% where the final temperature was $1000^{\circ} \mathrm{C}$. Values are based on original weight.

\begin{tabular}{|c|c|c|}
\hline Zeolite sample & Coating & Total weight loss $(\%)^{*}$ \\
\hline \multirow[t]{4}{*}{ LTA $4 \AA$} & Neat (uncoated) & 20.62 \\
\hline & SS & 21.13 \\
\hline & TMCS & 20.87 \\
\hline & Pyrene-Cl & 14.09 \\
\hline \multirow[t]{4}{*}{ LTA $5 \AA$} & Neat & 19.28 \\
\hline & SS & 20.45 \\
\hline & TMCS & 20.11 \\
\hline & Pyrene- $\mathrm{Cl}$ & 22.06 \\
\hline \multirow[t]{4}{*}{ NS1 } & Neat & 13.24 \\
\hline & Neat $\left(\mathrm{H}_{2} \mathrm{O}_{2}-\right.$ leached $)$ & 14.26 \\
\hline & $\mathrm{SS}$ & 17.32 \\
\hline & TMCS & 11.96 \\
\hline \multirow[t]{4}{*}{ NS2 } & Neat & 14.03 \\
\hline & Neat $\left(\mathrm{H}_{2} \mathrm{O}_{2}\right.$ - leached $)$ & 13.36 \\
\hline & $\mathrm{SS}$ & 13.86 \\
\hline & TMCS & 15.84 \\
\hline
\end{tabular}

${ }^{*}$ Values shown here are within $1 \%$ error. 
Table 4.2 Total weight loss of zeolite samples after exposure to solvent atmosphere. Values are based on original weight. For the NS1 and NS2 values, the reported literature value is given in parentheses.

\begin{tabular}{|c|c|c|c|c|}
\hline Zeolite sample & Coating & $\begin{array}{c}\text { Solvent } \\
\text { atmosphere }\end{array}$ & $\begin{array}{c}\text { Total weight } \\
\text { loss }(\%)\end{array}$ & $\begin{array}{c}\text { Final } \mathrm{T}\left({ }^{\circ} \mathrm{C}\right) \\
\text { isothermal for } 60 \\
\text { min }\end{array}$ \\
\hline \multirow[t]{4}{*}{ LTA $4 \AA$} & Neat (uncoated) & \multirow{4}{*}{$\mathrm{H}_{2} \mathrm{O}$} & $19.98 \pm 0.8$ & \multirow{4}{*}{300} \\
\hline & SS & & $19.25 \pm 0.3$ & \\
\hline & TMCS & & $20.16 \pm 0.9$ & \\
\hline & Pyrene-Cl & & $20.57 \pm 1.0$ & \\
\hline \multirow[t]{4}{*}{ LTA $4 \AA$} & Neat & \multirow{4}{*}{ Methanol } & $18.86 \pm 0.7$ & \multirow{4}{*}{300} \\
\hline & SS & & $18.11 \pm 1.1$ & \\
\hline & TMCS & & $18.64 \pm 0.8$ & \\
\hline & Pyrene-Cl & & $18.30 \pm 1.4$ & \\
\hline \multirow[t]{4}{*}{ LTA $4 \AA$} & Neat & \multirow{4}{*}{ Ethanol } & $19.95 \pm 0.6$ & \multirow{4}{*}{300} \\
\hline & SS & & $18.90 \pm 0.1$ & \\
\hline & TMCS & & $19.36 \pm 0.04$ & \\
\hline & Pyrene-Cl & & $19.06 \pm 0.1$ & \\
\hline NS1 & Neat & $\mathrm{H}_{2} \mathrm{O}$ & $12.57^{*}(16)$ & 450 \\
\hline NS2 & Neat & $\mathrm{H}_{2} \mathrm{O}$ & $9.99^{*}(17)$ & 450 \\
\hline
\end{tabular}

${ }^{*}$ Values shown here are within $1 \%$ error. 
Table 4.2 shows the weight loss of LTA $4 \AA$ that was exposed to three solvents: water, methanol and ethanol. It could be stated that, in general, the coated materials in all three solvent atmospheres exhibited a tendency to take up less of the solvent molecules than in the neat zeolite case. This would suggest that the coating material inhibits the uptake of solvent, acting as a pore-blocker, where the neat sample has no such protection. Methanol and ethanol in comparison to water show only slightly less of an affinity for the zeolite, whether the sample is coated or not. Water is easily taken up by the zeolite structure, as water molecules are small enough to pass through the apertures and diffuse through the channels and cages of the zeolite and tend to surround cations that reside in the zeolite structure due to electrostatic attraction. Methanol and ethanol do not share the same properties as water does, being less polar. In addition to polarity differences among the three solvents, there is also a difference in size (Fig. 4.1, 4.2). Water is not hindered by its size to enter the LTA $4 \AA$ zeolite structure and can enter the zeolite structure regardless of orientation, as is the case with methanol. Conversely, ethanol must be properly oriented, such that the molecule should face the apertures of the zeolite structure in an "end-on" orientation to guarantee diffusion into the zeolite. A "face-on" entrance is not possible, as the length of ethanol from tip to tail is greater than $4 \AA$. The calculation of the length of the ethanol molecule, as well as for water and methanol was based on the addition of standard bond lengths of: $\mathrm{C}-\mathrm{H}, \mathrm{C}-\mathrm{C}, \mathrm{C}-\mathrm{O}$ and $\mathrm{O}-$ $\mathrm{H}$ as are provided in classical texts. In the case of the LTA $5 \AA$, all three solvents can enter 


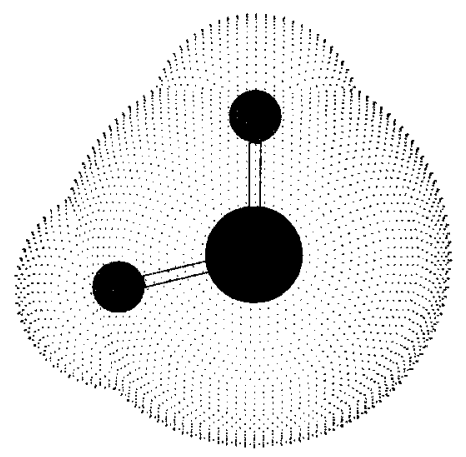

(a)
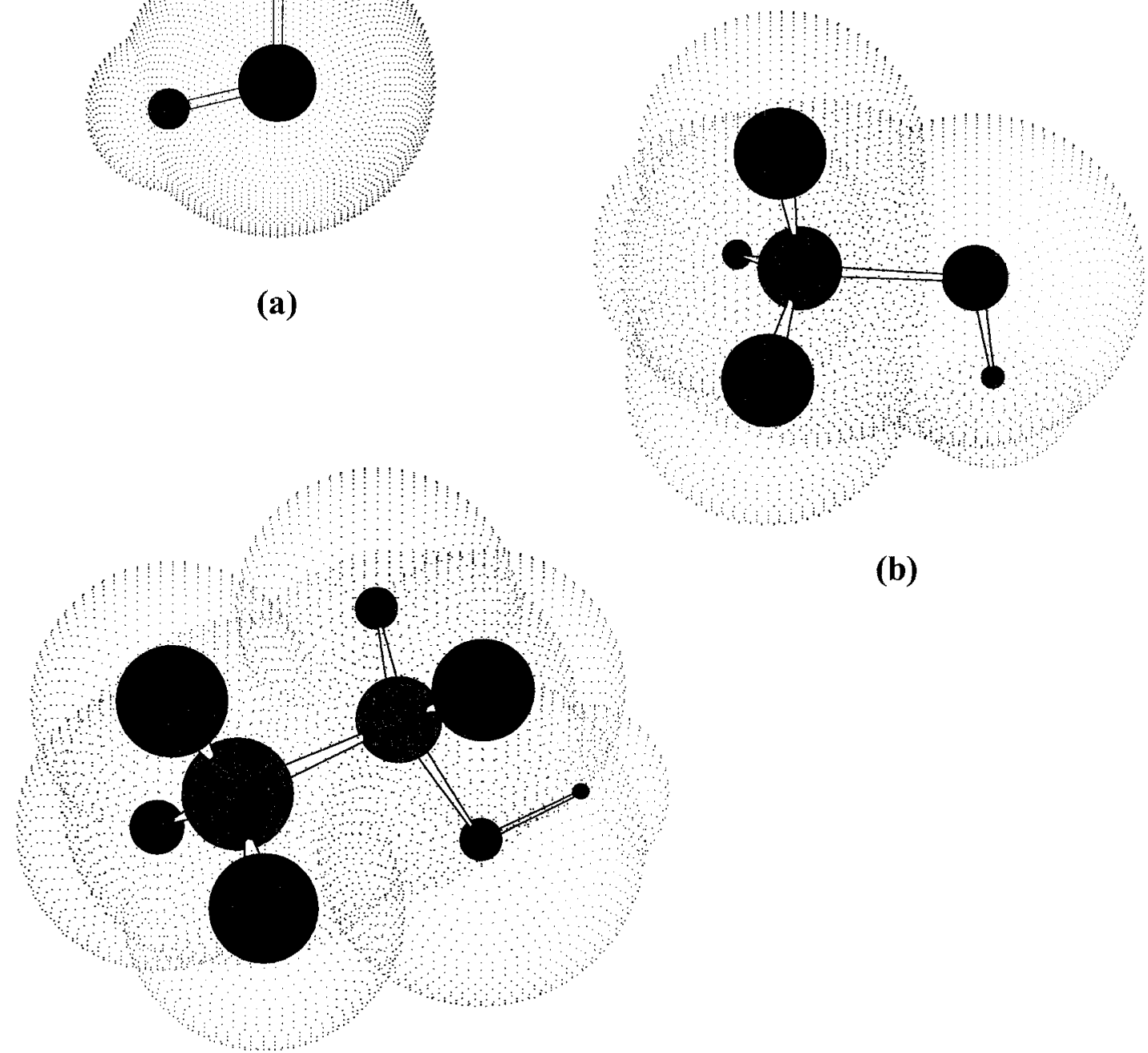

(b)

(c)

Fig. 4.1 Ball and stick models of : a, water $(\sim 1.5 \AA$ length; $b$, methanol $(\sim 3.5$ $\AA$ length); c, ethanol $(\sim 4.5 \AA$ length $)$. Molecules shown are to illustrate how slight difference in size can alter diffusion into a zeolite. 

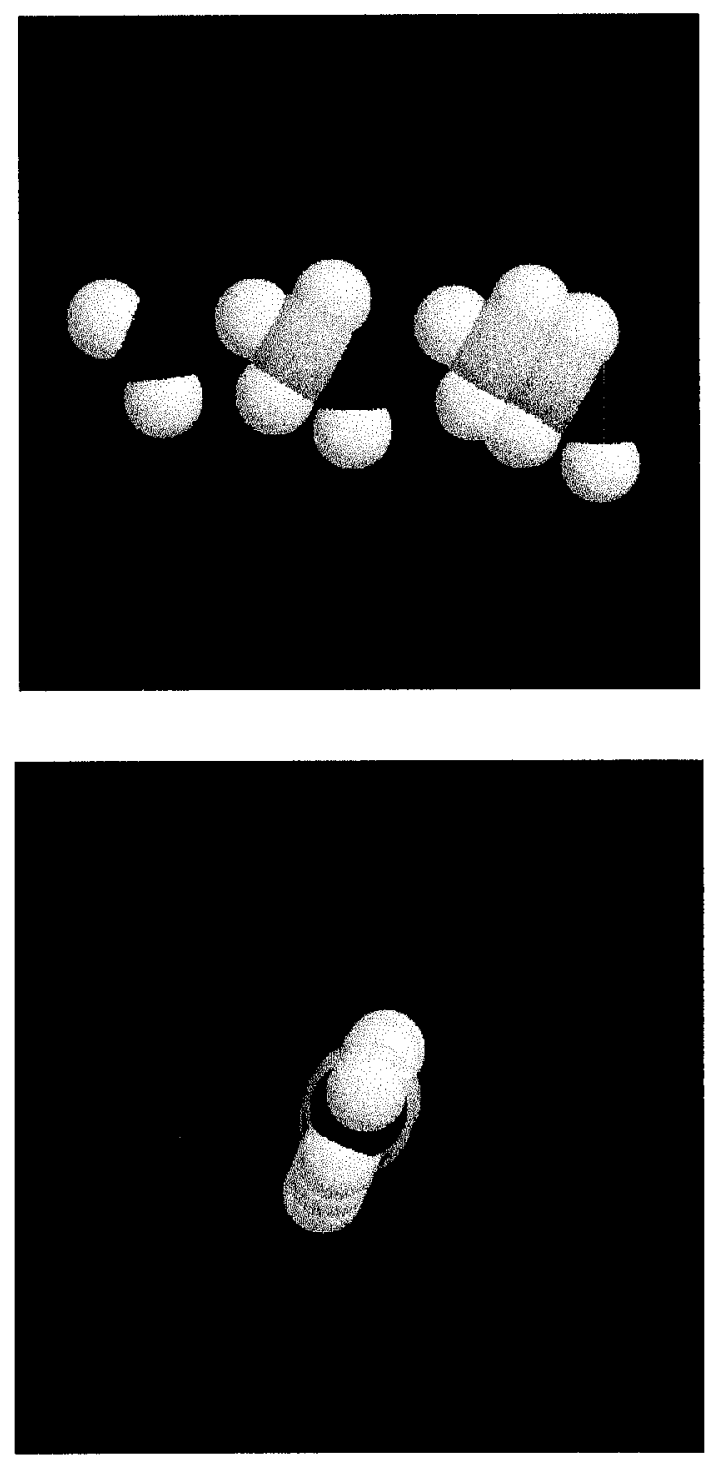

Fig. 4.2 Space filling models of water, methanol and ethanol molecules. The top diagram illustrates the difference in length; from left to right: water, methanol, ethanol. The bottom diagram shows a side view, where the cross-sectional diameter of all three molecules is similar. 
the zeolite structure, as the apertures are large enough to accommodate the molecules, again, regardless of orientation. The versatility of the zeolite samples with respect to the solvent molecules is evident in that the weight percent uptake does not greatly differ from water to methanol and finally to ethanol.

The NS1 and NS2 samples were heated to $450^{\circ} \mathrm{C}$ to determine total water loss of these natural samples. Literature values ${ }^{1}$ show an increased weight loss due to the evolution of water from the zeolite structure in comparison to the experimental values shown in Table 4.2. Given that these are natural samples, and not purified to remove mineral impurities, it would be expected that those impurities such as quartz, mica or feldspar do not have water uptake capabilities, due to their mineral structure devoid of pores and channels. This would, therefore, give the sample less water uptake overall.

\subsection{Cycle experiments}

These experiments were carried out to document some unusual observations, the fact that weight was regained on cooling the samples. Table 4.3 summarizes the initial weight loss due to water and the subsequent uptake of flow gas for all zeolite samples. Table 4.4 summarizes the average gain and loss of flow gas for all zeolite samples. All the samples were placed in a sealed container, exposed to an atmosphere of water vapour. During analysis, nitrogen was employed as the flow gas. In general, all materials exhibited repeatable weight loss with the increase of temperature, and a weight gain with the decrease of temperature. In the initial weight loss, water is driven off. When the temperature is 
decreased, weight is gained, indicating the uptake of nitrogen. The cycle is repeated, where weight loss occurs with temperature increase, and conversely, weight gain with temperature decrease. A sample TGA plot is shown in Fig. 4.3. It is not thought that water is being readsorbed into the zeolite structure, as a constant flow of nitrogen, from a cylinder containing nitrogen at $99.998 \%$ purity, is maintained throughout the cycle. The most $\mathrm{N}_{2}$ uptake is observed in the $4 \AA$ and $5 \AA$ samples coated with the Pyrene-Cl coating. Since this is a relatively large molecule in comparison to SS and TMCS, it is thought that when the sample is being cooled, $\mathrm{N}_{2}$ that is adsorbed into the zeolite structure might also be trapped under the coating material as it bends towards the surface of the zeolite at lower temperatures. In the cases of NS1 and NS2, this idea does not hold true, as the TMCS coated versions lost more initial water and gained more $\mathrm{N}_{2}$. This allows for postulation of whether the smaller, less flexible coatings have the ability to trap sorbent molecules just underneath the methyl groups that are substituents of the silicon moiety bound to the zeolite surfaces (Fig. 4.4).

In addition to cycle experiments with nitrogen, it was of interest to take a look at the uptake of LTA $4 \AA$ and $5 \AA$, neat, in a cycle experiment with helium as the flow gas (Fig. 4.5). It was observed that a substantial initial uptake of $14.99 \%$ for $4 \AA$ and $13.94 \%$ for $5 \AA$ for the case of helium in contrast to the $6 \%$ uptake for nitrogen. Helium atoms are much smaller and can diffuse much more easily into the into the $\beta$ cages of the zeolite structure; conversely, nitrogen is only able to diffuse into the $\alpha$ cages. 
Table 4.3 Initial water loss and initial gain of gas during the first cycle of temperature cycle experiments, where the maximum temperature was $350^{\circ} \mathrm{C}$, and the minimum $25^{\circ} \mathrm{C}$. Values are based on original weight.

\begin{tabular}{|c|c|c|c|c|c|}
\hline \multirow{2}{*}{$\begin{array}{l}\text { Zeolite } \\
\text { sample }\end{array}$} & \multirow[t]{2}{*}{ Coating } & \multicolumn{2}{|c|}{ Initial $\mathrm{H}_{2} \mathrm{O}$ loss $(\%)^{*}$} & \multirow{2}{*}{$\begin{array}{l}\text { Initial } \mathrm{N}_{2} \\
\text { gain }(\%)^{*}\end{array}$} & \multirow{2}{*}{$\begin{array}{c}\text { Initial He gain } \\
(\%) \\
\end{array}$} \\
\hline & & $\mathrm{N}_{2}$ flow & He flow & & \\
\hline \multirow[t]{4}{*}{ LTA $4 \AA$} & Neat (uncoated) & 19.27 & 19.78 & 6.22 & $14.22 \pm 1.10$ \\
\hline & SS & 20.09 & & 5.73 & \\
\hline & TMCS & 19.93 & & 4.84 & \\
\hline & Pyrene-Cl & 19.02 & & 9.80 & \\
\hline \multirow[t]{4}{*}{ LTA $5 \AA$} & Neat & 20.15 & 19.26 & 5.66 & $13.44 \pm 0.71$ \\
\hline & SS & 19.51 & & 5.21 & \\
\hline & TMCS & 19.10 & & 6.08 & \\
\hline & Pyrene-Cl & 20.71 & & 6.66 & \\
\hline \multirow[t]{3}{*}{ NS1 } & Neat & 11.30 & & 4.32 & \\
\hline & $\mathrm{SS}$ & 11.90 & & 4.35 & \\
\hline & TMCS & 12.89 & & 4.72 & \\
\hline \multirow[t]{3}{*}{ NS2 } & Neat & 9.31 & & 2.32 & \\
\hline & SS & 9.62 & & 2.56 & \\
\hline & TMCS & 10.29 & & 2.74 & \\
\hline
\end{tabular}

${ }^{*}$ Values shown here are within $1 \%$ error. 
Table 4.4 Average water loss and average gain of gas during temperature cycle experiments, where the maximum temperature was $350^{\circ} \mathrm{C}$, and the minimum $25^{\circ} \mathrm{C}$. Values are based on original weight.

\begin{tabular}{|c|c|c|c|}
\hline $\begin{array}{l}\text { Zeolite } \\
\text { sample }\end{array}$ & Coating & Average $\mathrm{N}_{2}$ gain (\%) & Average $\mathrm{N}_{2}$ loss $(\%)$ \\
\hline \multirow[t]{4}{*}{ LTA $4 \AA$} & Neat (uncoated) & $5.83 \pm 0.55$ & $5.85 \pm 0.56$ \\
\hline & SS & $5.39 \pm 0.48$ & $5.43 \pm 0.49$ \\
\hline & TMCS & $4.56 \pm 0.39$ & $4.59 \pm 0.42$ \\
\hline & Pyrene-Cl & $9.01 \pm 1.12$ & $9.04 \pm 1.14$ \\
\hline \multirow[t]{4}{*}{ LTA $5 \AA$} & Neat & $4.72 \pm 1.31$ & $4.72 \pm 1.32$ \\
\hline & SS & $4.83 \pm 0.64$ & $4.91 \pm 0.69$ \\
\hline & TMCS & $5.01 \pm 0.57$ & $5.08 \pm 0.62$ \\
\hline & Pyrene- $\mathrm{Cl}$ & $5.85 \pm 1.04$ & $5.93 \pm 1.06$ \\
\hline NS1 & Neat & $4.05 \pm 0.25$ & $4.07 \pm 0.29$ \\
\hline NS2 & Neat & $2.28 \pm 0.22$ & $2.30 \pm 0.23$ \\
\hline
\end{tabular}




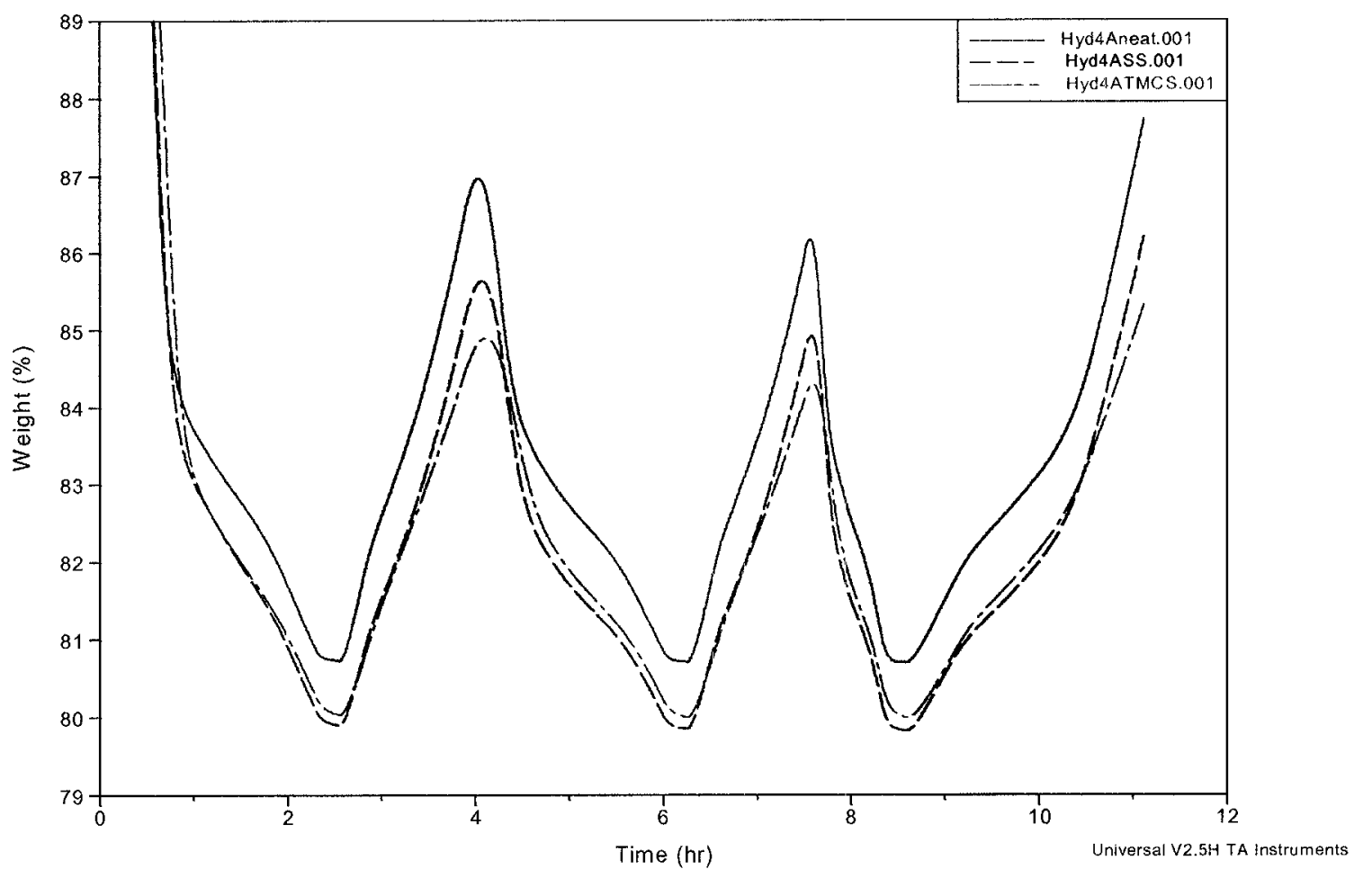

Fig. 4.3 Cycle experiment for zeolite LTA $4 \AA$. The green curve is the neat sample, the blue is SS-coated sample, and the red is the TMCS-coated sample. Where the curves show a decrease in mass, the temperature is being increased to $350{ }^{\circ} \mathrm{C}$; conversely, where the curve shows an increase, the temperature is being decreased to $25^{\circ} \mathrm{C}$. 


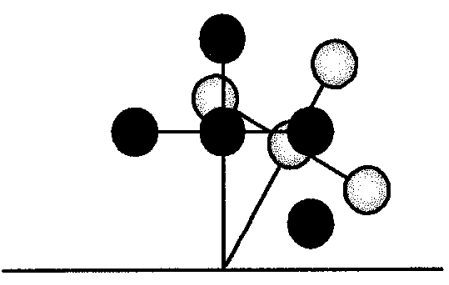

(a)

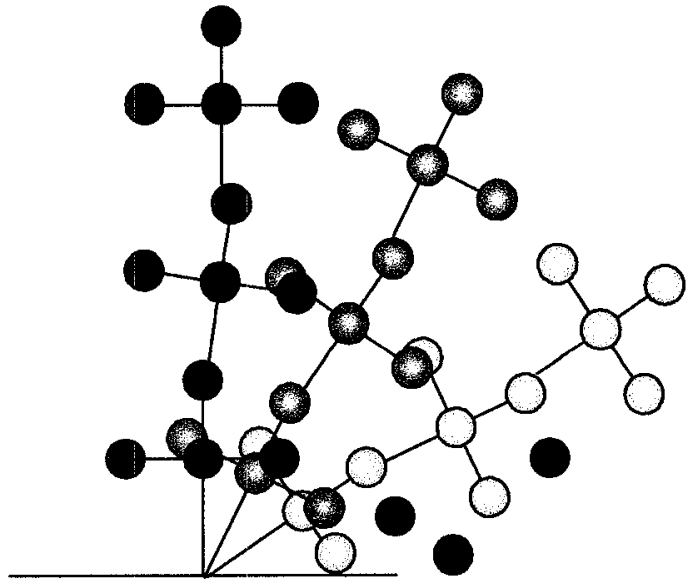

(b)

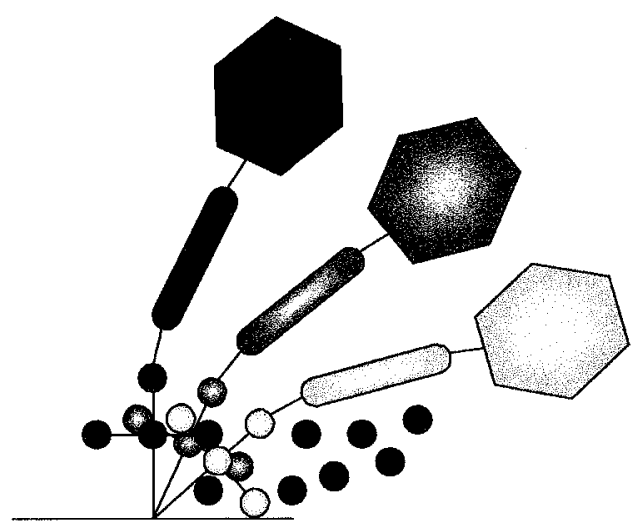

(c)

Fig. 4.4 Cartoon representation of the possible behaviour of the coating material, and the suspected ability to trap water or other solvent molecules (small blue spheres). 


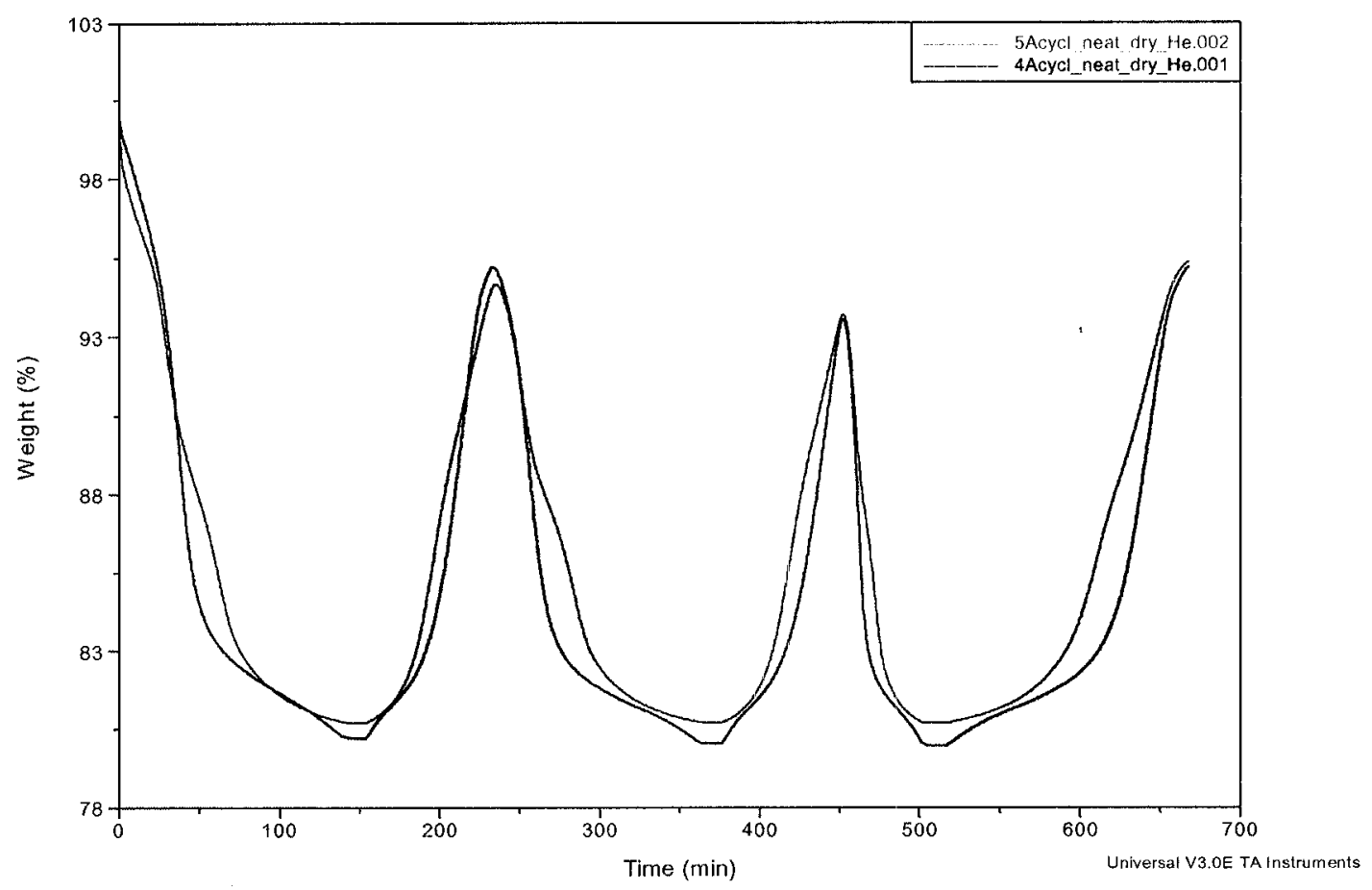

Fig. 4.5 TGA plot of a cycle experiment with LTA $4 \AA$ (blue curve) and $5 \AA$ (red curve) with helium as the flow gas. The temperature range was from $25^{\circ} \mathrm{C}$ to $350^{\circ} \mathrm{C}$. Weight loss denotes temperature increase; conversely, weight gain denotes temperature decrease. 


\section{Chapter IV Results and Discussion}

\section{Porosimetry}

The natural, neat samples of NS1 and NS2 were subjected to porosimeter analysis to determine the available surface area and pore size and volume. The data for these samples is summarized in Table 4.5. MOR has reported ${ }^{2}$ pore sizes of $6.5 \times 7.0 \AA$ and $2.6 \times 5.7 \AA$, and it would be expected to see a value around these figures in the porosimeter analysis. The values calculated for the average adsorption and desorption pore diameters for NS1 are given as 213 and $68 \AA$ respectively. Were this a sample of pure mordenite, it would be expected to see a value around that of the literature values, and even were there organic matter trapped in the zeolite structure, blocking pores and channels, one would expect a decrease in pore size. The pore volume of NS1 has been reported ${ }^{3}$ to $b e .26 \mathrm{~cm}^{3} / \mathrm{g}$, whereas the experimental data reports a value of about $0.015 \mathrm{~cm}^{3} / \mathrm{g}$. This decrease in pore volume would support the presence of organic impurities that might be present in the zeolite. Surface area calculations have given values of less than $20 \mathrm{~m}^{2} / \mathrm{g}$, which is quite low, as molecular sieves with the same pore diameter have surface areas around and above $100 \mathrm{~m}^{2} / \mathrm{g} .{ }^{4}$ The same argument can be made for the natural NS2 sample, where the calculated pore diameter is larger than the pore dimensions reported in the literature ${ }^{2}(6.1 \times 4.9 \AA)$. Calculated pore volumes are also low, around $0.007 \mathrm{~cm}^{3} / \mathrm{g}$. Surface area calculations give values less than $10 \mathrm{~m}^{2} / \mathrm{g}$. These discrepancies could be attributed to mineral impurities, again, such as quartz, feldspar and mica, with the absence of internal porosity. These additional minerals might have undergone erosion to having "pits" form on the surfaces, perhaps giving inflated pore diameter readings. 
Table 4.5 Porosimeter data for NS1 and NS2, showing calculated surface area, pore diameter and pore volume.

\begin{tabular}{|c|c|c|}
\hline Measured Parameter & NS1 & NS2 \\
\hline $\begin{array}{l}\text { Single point surface area at } \mathrm{P} / \mathrm{P}_{\mathrm{o}} \\
\left(\mathrm{m}^{2} / \mathrm{g}\right)( \pm 0.05)\end{array}$ & 12.53 & 4.92 \\
\hline BET surface area $\left(\mathrm{m}^{2} / \mathrm{g}\right)( \pm 0.05)$ & 12.19 & 5.02 \\
\hline Langmuir surface area $\left(\mathrm{m}^{2} / \mathrm{g}\right)( \pm 0.05)$ & 18.02 & 7.20 \\
\hline $\begin{array}{l}\text { BJH Adsorption Cumulative Surface } \\
\text { Area of Pores }\left(\mathrm{m}^{2} / \mathrm{g}\right)( \pm 0.05)\end{array}$ & 2.96 & 2.02 \\
\hline $\begin{array}{l}\text { BJH Desorption Cumulative Surface } \\
\text { Area of Pores }\left(\mathrm{m}^{2} / \mathrm{g}\right)( \pm 0.05)\end{array}$ & 8.63 & 3.22 \\
\hline $\begin{array}{l}\text { BJH Adsorption Cumulative Volume } \\
\text { of Pores }\left(\mathrm{cm}^{3} / \mathrm{g}\right)( \pm 0.05)\end{array}$ & 0.016 & 0.008 \\
\hline $\begin{array}{l}\text { BJH Desorption Cumulative Volume } \\
\text { of Pores }\left(\mathrm{cm}^{3} / \mathrm{g}\right)( \pm 0.005)\end{array}$ & 0.015 & 0.007 \\
\hline $\begin{array}{l}\text { BJH Adsorption Average Pore } \\
\text { Diameter }(\AA)( \pm 0.05)\end{array}$ & 212.99 & 156.81 \\
\hline $\begin{array}{l}\text { BJH Desorption Average Pore } \\
\text { Diameter }(\AA)( \pm 0.05)\end{array}$ & 68.26 & 80.81 \\
\hline $\begin{array}{l}\text { Horvath-Kawazoe Maximum Pore } \\
\text { Volume }\left(\mathrm{cm}^{3} / \mathrm{g}\right)( \pm 0.005)\end{array}$ & 0.006 & 0.002 \\
\hline $\begin{array}{l}\text { Horvath-Kawazoe Median Pore } \\
\text { Diameter }(\AA)( \pm 0.05)\end{array}$ & 6.54 & 8.03 \\
\hline Time outgassed (days) & 3 & 2.5 \\
\hline Temperature outgassed $\left({ }^{\circ} \mathrm{C}\right)$ & 300 & 300 \\
\hline
\end{tabular}


These minerals may also contribute to low surface areas, as only external surface does not provide much area for adsorption as would a zeolite that possesses both internal and external available surface. In addition to this, the pore sizes determined have no relation to the internal micropores of the minerals and are most likely related to the sample morphology.

\section{Powder X-Ray diffraction (PXRD) of the natural zeolite samples}

The use of PXRD allowed for powder patterns to be obtained for the natural zeolite samples. Direct comparison using the JCPDS database did not aid in the verification of the NS1 and NS2 samples. Fig. 4.6 shows the NS1 powder pattern compared to the JCPDS file, indicated by red, narrow lines superimposed on the raw data file. Comparing the ray pattern for NS1 to the JCPDS pattern for MOR, there seems to be no direct correlation of the peaks to be able to make a definite statement as to the identity of the natural sample as being MOR. Many impurities are present in the sample; however, sample purity was not assessed. A few peaks at low angle match up between the two patterns; however, this hardly constitutes a match. NS1 contains impurities, either of organic or inorganic nature. A search and match sequence was done to determine to which zeolite powder pattern NS1 most closely correlates; unfortunately, no match was found. The sample can be said to possess crystal character, as the peaks are sharp; hence, glassy, amorphous materials are not major constituents of the sample matrix.

Similarly, in the case of NS2, on being compared to the JCPDS pattern (Fig. 4.7), the same statements can be made as to the gross mis-matching between the raw and the database 
patterns. In the case of NS2, there seems to be a closer overlapping of the peaks than in the former example, but again, no definite conclusion can be made as to the identity of the sample, as no JCPDS database patterns were able to match exactly. 


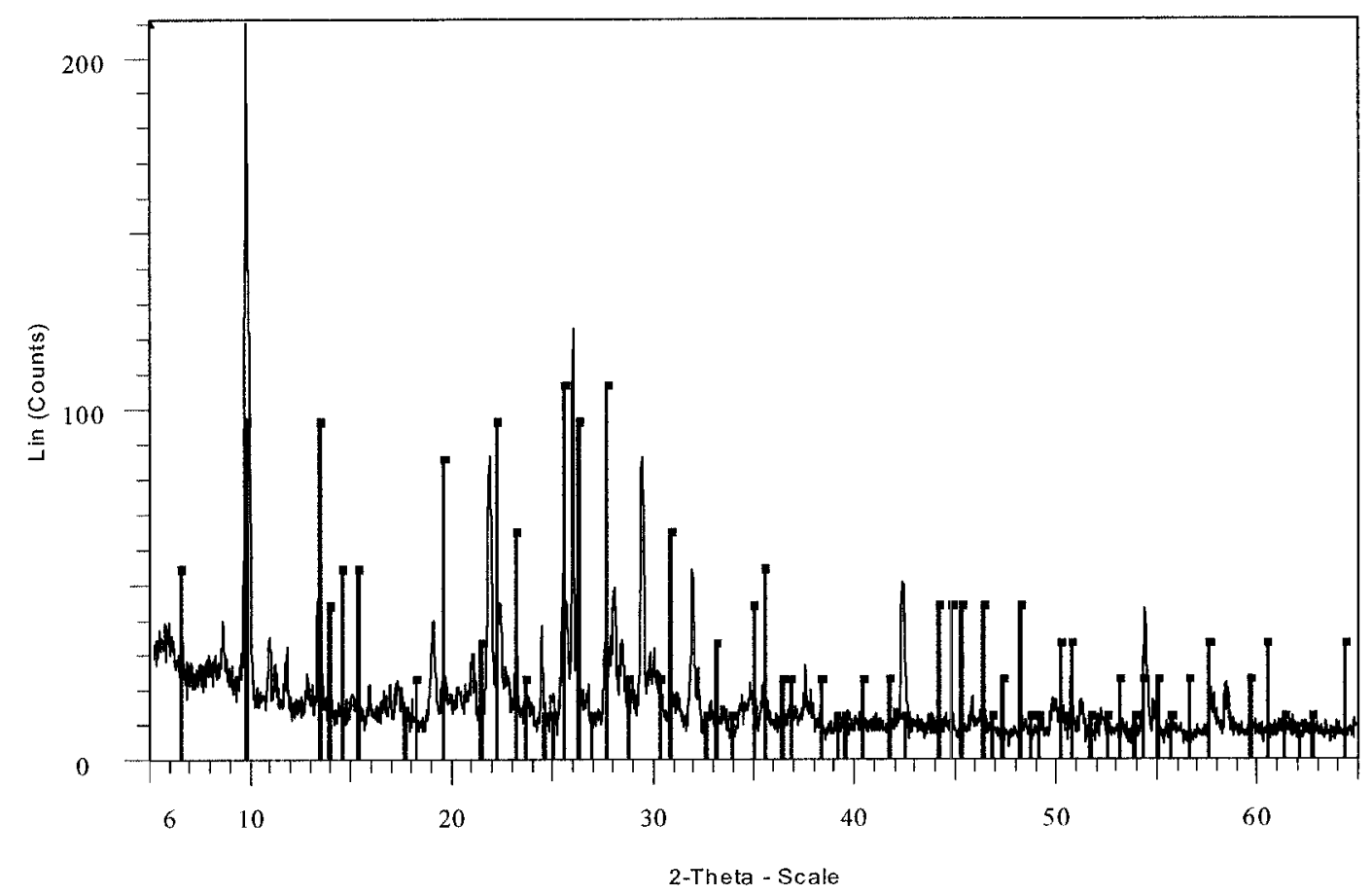

M5, 31.2 - File: mord1.raw - Type: 2 Th/Th locked - Start: $5.020^{\circ}$ - End: $65.000^{\circ}$ - Step: $0.020^{\circ}$ - Step time: [06-0240 (D) - Mordenite - (Ca,Na2,K2)Al2Si10024-7H2O - Y: 50.00\% - dx by: 1. - WL: 1.5406 - Orthorho

Fig 4.6 PXRD pattern of NS1; JCPDS peaks are shown in red. 


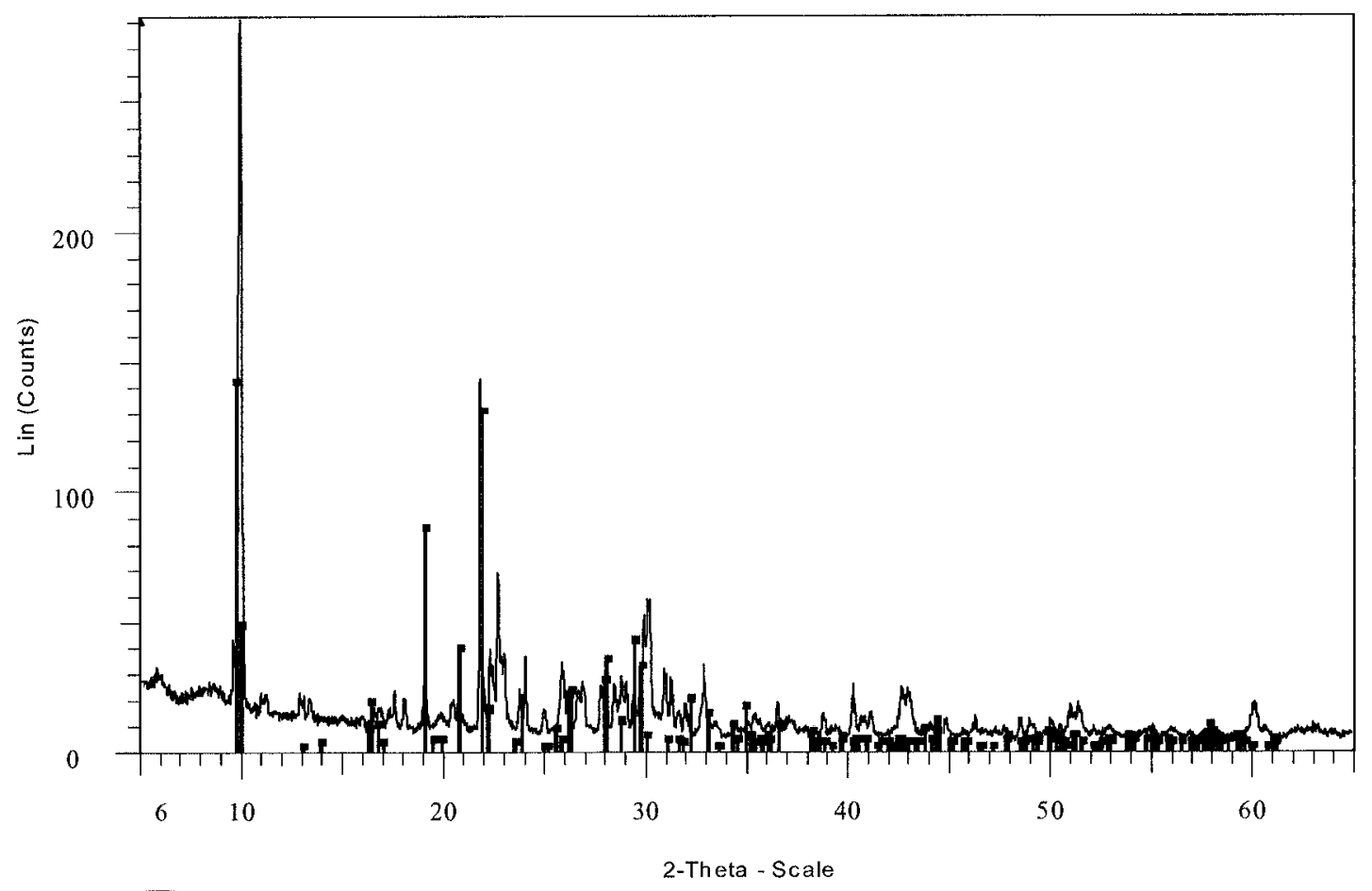

W5, 25.27 - File: stib1 raw - Type: 2 Th/Th locked - Start: $5.020^{\circ}$ - End: $65.000^{\circ}$ - Step: $0.020^{\circ}$ - Step time: 79m1676 (C) - Stilbite - Na.72Ca4(Al10Si26072)(H2O)29.12 - Y: $50.00 \%$ - d x by: 1. - WL: 1.5406 - Orthor

Fig 4.7 XRD pattern of NS2; JCPDS peaks a re shown in red. 


\section{Solid State Nuclear Magnetic Resonance (SS NMR) Spectroscopy}

This technique allowed observation of chemical environments of three distinct nuclei:

${ }^{13} \mathrm{C}$, in coating material present on the zeolite surface, ${ }^{29} \mathrm{Si}$, to attempt to determine sample purity and identity of NS1 and NS2; and ${ }^{27} \mathrm{Al}$, to verify the coordination of Al nuclei in the NS1 and NS2 zeolite samples.

\section{1 ${ }^{13}$ C SS NMR spectroscopy of coated LTA $4 \AA$}

Fig. 4.8 shows the ${ }^{13} \mathrm{C}$ SS MAS spectrum for LTA $4 \AA$, surface modified with SS. Methyl carbons that are adjacent to silicon are characteristically shown to have a chemical shift at around 0 ppm; this spectrum confirms their presence. SS has only methyl carbons, so additional peaks should not be present in the spectrum. The large, broad peak that is observed is attributed to carbon signals in the polyethylene spinner assembly surrounding the sample. What is of interest here is the peak at the 0 ppm region, which confirms the presence of the coating material.

\section{2 ${ }^{29}$ Si SS NMR spectroscopy of the natural zeolite samples}

Solid-state NMR spectroscopy, specifically that of the ${ }^{29} \mathrm{Si}$ nucleus, was performed to determine the chemical environment of the Si in the NS1 and NS2 natural samples. ${ }^{29} \mathrm{Si}$ SS NMR can also be used as a zeolite identification tool. MOR and STI are shown, in the literature $^{5}$, to possess three and four chemically inequivalent Si species with resonances at $-100.1,-105.7$ and $-112.1 \mathrm{ppm}(\mathrm{MOR}) ;-98,-101.5+-103.6$ and $-108 \mathrm{ppm}$ (STI). These peaks correspond to the $\mathrm{Si}(2 \mathrm{Al}), \mathrm{Si}(1 \mathrm{Al})$ and $\mathrm{Si}(0 \mathrm{Al})$ chemical environments, respectively. 


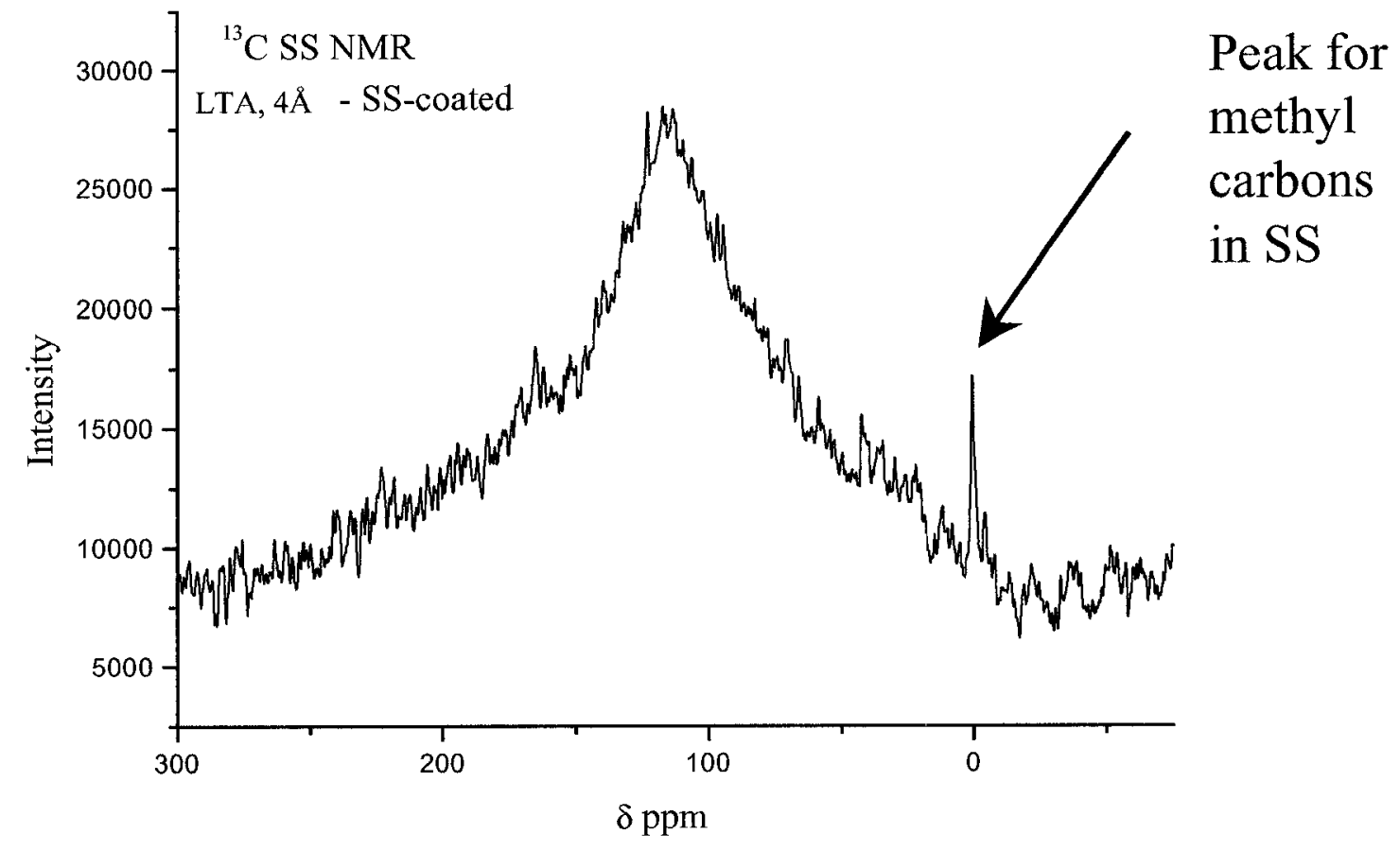

Fig 4.8 $\quad{ }^{13} \mathrm{C}$ SS NMR spectrum of SS-coated zeolite LTA $4 \AA$. 


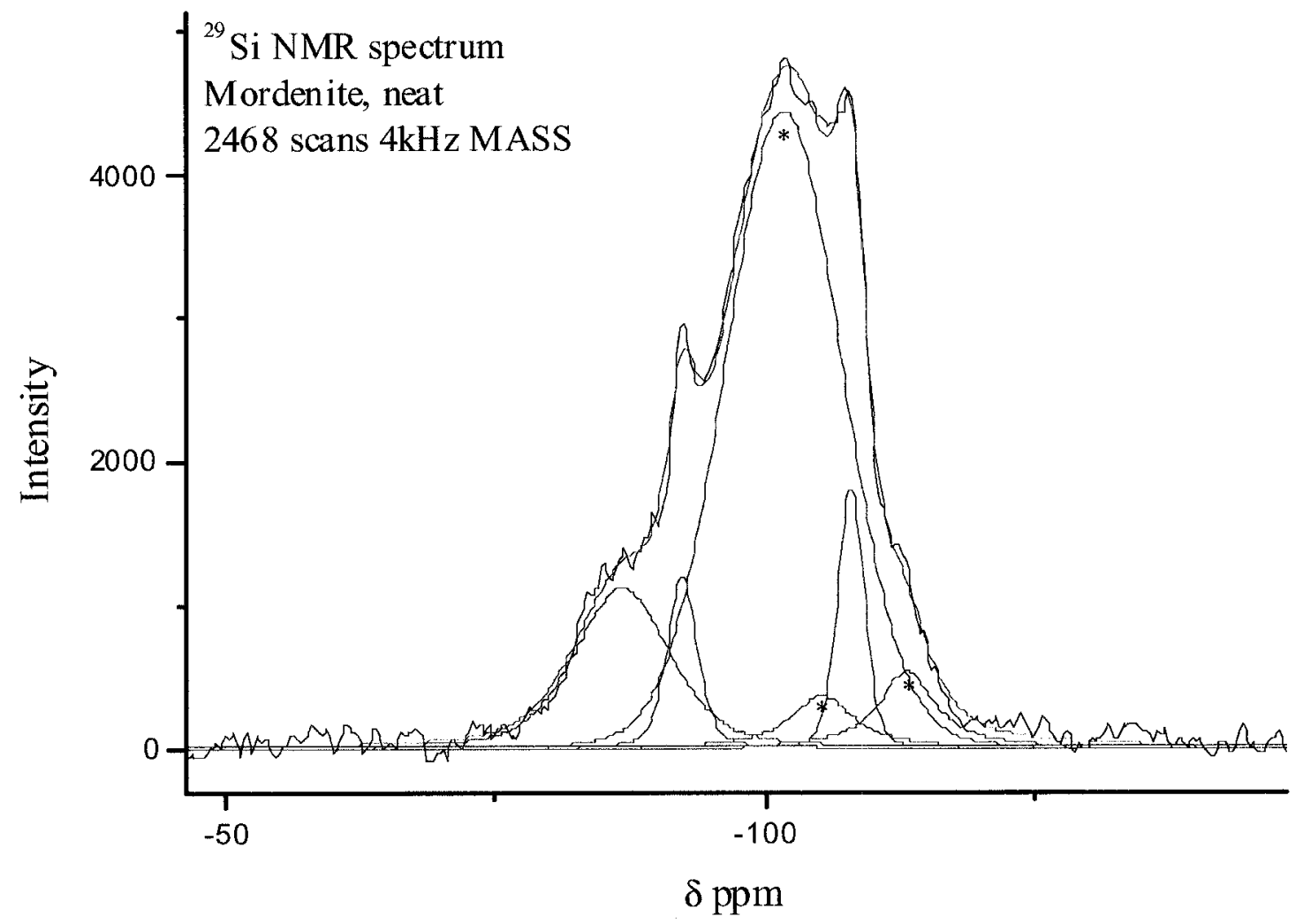

Fig 4.9 ${ }^{29}$ Si SS NMR spectrum of NS1. Asterisks denote the peaks that closely match the literature values. 


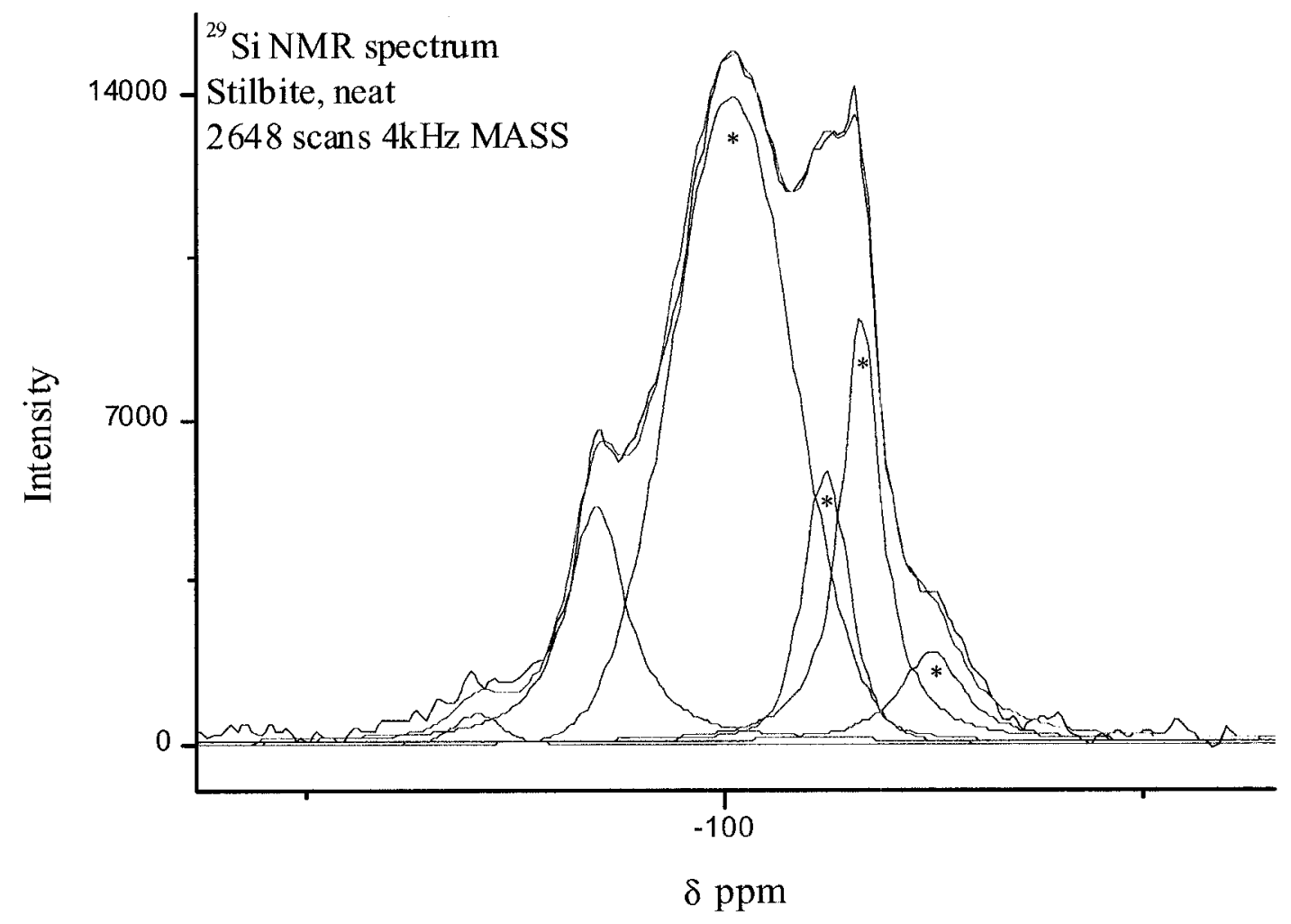

Fig 4.10 ${ }^{29}$ Si SS NMR spectrum of NS2. Asterisks denote the peaks that closely match the literature values. 
The spectra for NS1 (Fig. 4.9) and NS2 (Fig. 4.10) show that there exist, within the sample, all 5 chemical environments, represented by 6 peaks in both NS1 and NS2. The extra peaks suggest mineral impurities, and correlate with the same inference derived from the appearance of extra peaks that appear in the PXRD patterns. The evolution of a sixth peak in these cases does not imply a sixth chemical environment, but probably indicates a crystallographic inequivalence between chemically equivalent Si nuclei. This sixth peak can be assigned to a mineral impurity, as the $\mathrm{Si}-\mathrm{O}-\mathrm{Al}$ angle or bond length must be different from that which is present in the zeolite structure.

Employing the equation:

$$
S i / A l=\sum_{n=0}^{4} I_{S i(n A l)} / \sum_{n=0}^{4} \frac{n}{4} I_{S i(n A l)}
$$

the $\mathrm{Si}: \mathrm{Al}$ ratio can be determined, which is characteristic of a particular zeolite structure. Using the peaks in the spectra that match most closely to those in the literature, the Si:Al ratios for NS1 and NS2 were calculated to be 1.94 and 2.26 respectively, compared to 4.4-5.5 (MOR) and 2.6 (STI). It is evident that the ${ }^{29} \mathrm{Si} \mathrm{NMR} \mathrm{results} \mathrm{of} \mathrm{NS1} \mathrm{show} \mathrm{even} \mathrm{more}$ disparity from those of the known values for MOR, and than those from PXRD. NS2 results show more similarity towards those of the STI values given; however, again, no significant match is found. Table 4.6 shows the peak values obtained for these minerals with the literature values, and the corresponding chemical environment for ${ }^{29} \mathrm{Si}$. 
Table 4.6 ${ }^{29}$ Si experimental chemical shifts of NS1 and NS2 in comparison to the literature values. Chemical shifts are correct to within $10 \%$ error.

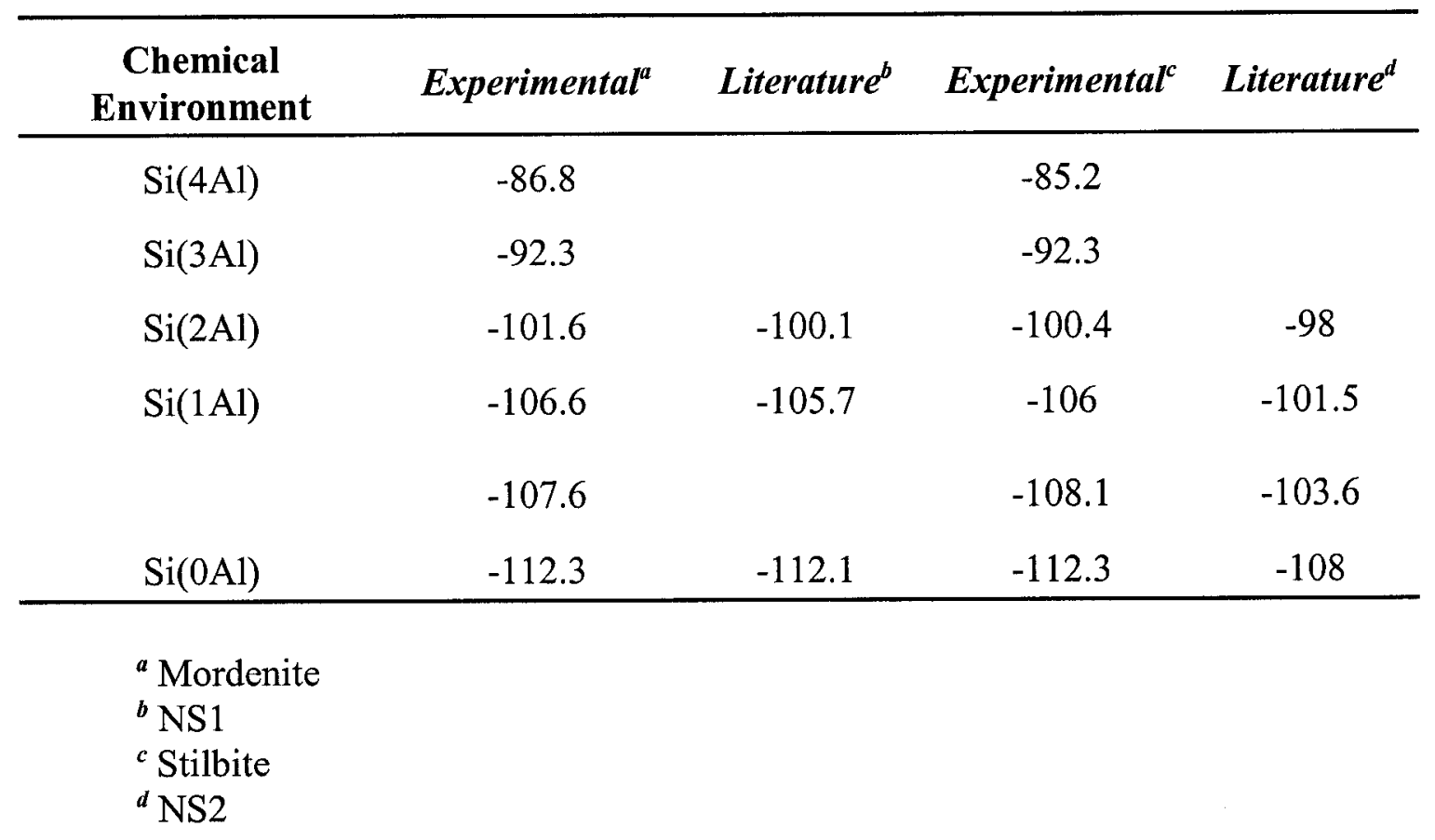



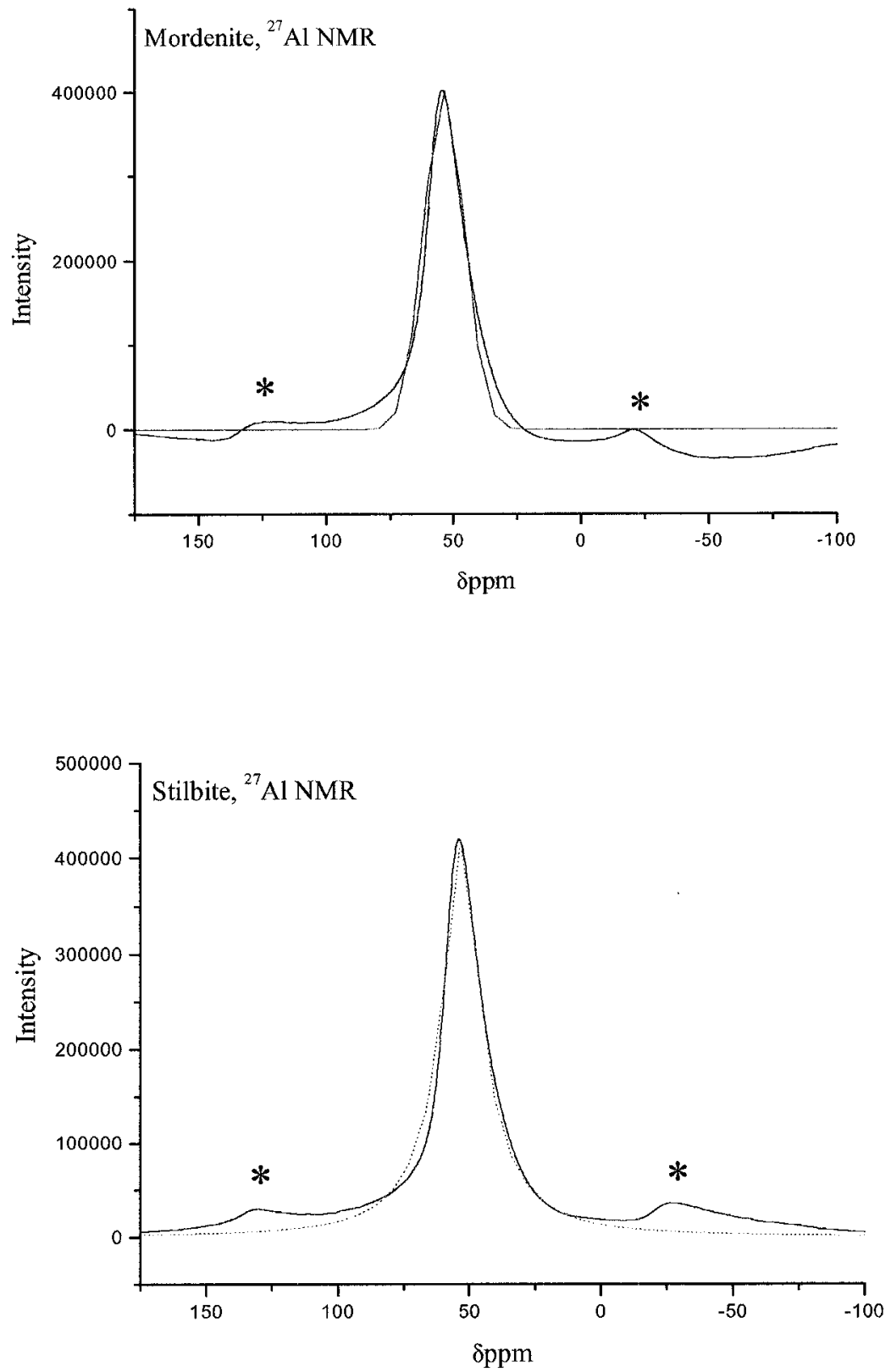

Fig 4.11 ${ }^{27}$ Al SS NMR spectra of NS1 (top) and NS2 (bottom). Spinning sidebands are shown with asterisks. 


\section{3 ${ }^{27}$ Al SS NMR spectroscopy of the natural zeolite samples}

${ }^{27} \mathrm{Al}$ SS NMR spectroscopy of NS1 and NS2 were performed to determine the chemical coordination of the Al nucleus in the zeolite structures. Both NS1 and NS2 give clean, simple spectra with one peak at approximately $50 \mathrm{ppm}$, the value that corresponds to tetrahedrally coordinated Al. NS1 is reported ${ }^{5}$ to have a ${ }^{27} \mathrm{Al}$ chemical shift at $55.8 \mathrm{ppm}$. No other environments for $\mathrm{Al}$ are present in the samples; that is, no octahedrally coordinated, or free aluminum is in the zeolite structure. Fig 4.11 shows the ${ }^{27} \mathrm{Al}$ spectra for NS1 and NS2; spinning sidebands are denoted with asterisks.

\section{Uptake experiments}

Duplicates of Linde $4 \AA$ and Linde $5 \AA$ of both the neat and coated samples were placed in sealed containers with one of three solvents: water $(97 \%$ humidity maintained with $\mathrm{a} \mathrm{K}_{2} \mathrm{SO}_{4}$ brine solution), methanol or ethanol. It was intended to observe the rate of uptake of these samples as well as to compare maximum uptake of solvent, by allowing the sample to become saturated by the solvent in its vapour phase. Figures 4.12 to 4.14 illustrate how the rate of uptake is similar for all samples in each solvent atmosphere, show overlapping regions of error, and all reach a plateau of uptake at 15 hours of exposure time. Again, this overlap, suggests that all the zeolites, coated and uncoated, exhibit the same amount of uptake capability in each of the solvent atmospheres. No real trend can be established as to pattern of uptake among the coated materials and the neat samples; however, it is observed that there is a larger uptake of water in comparison to that of methanol and ethanol 
Linde 4A, 97\% Water Atmosphere

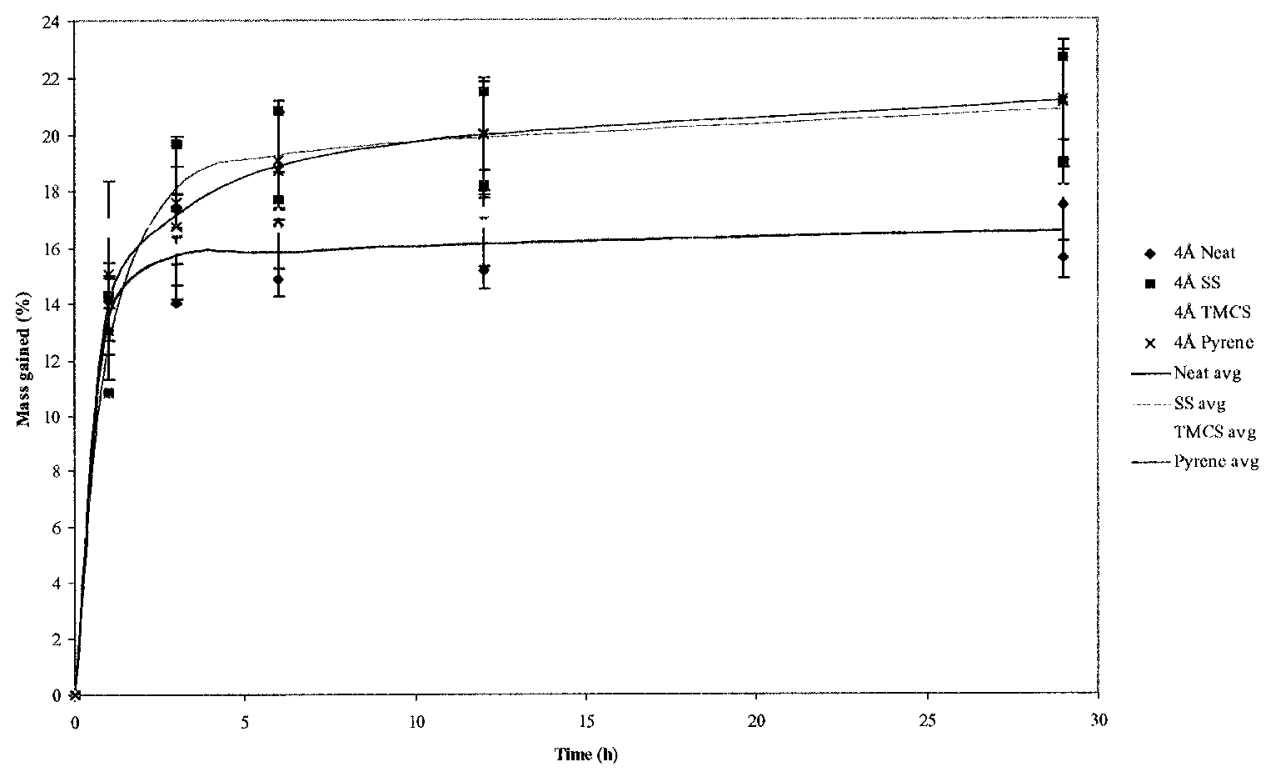

Linde $5 \hat{A}, 97 \%$ Water Atmosphere

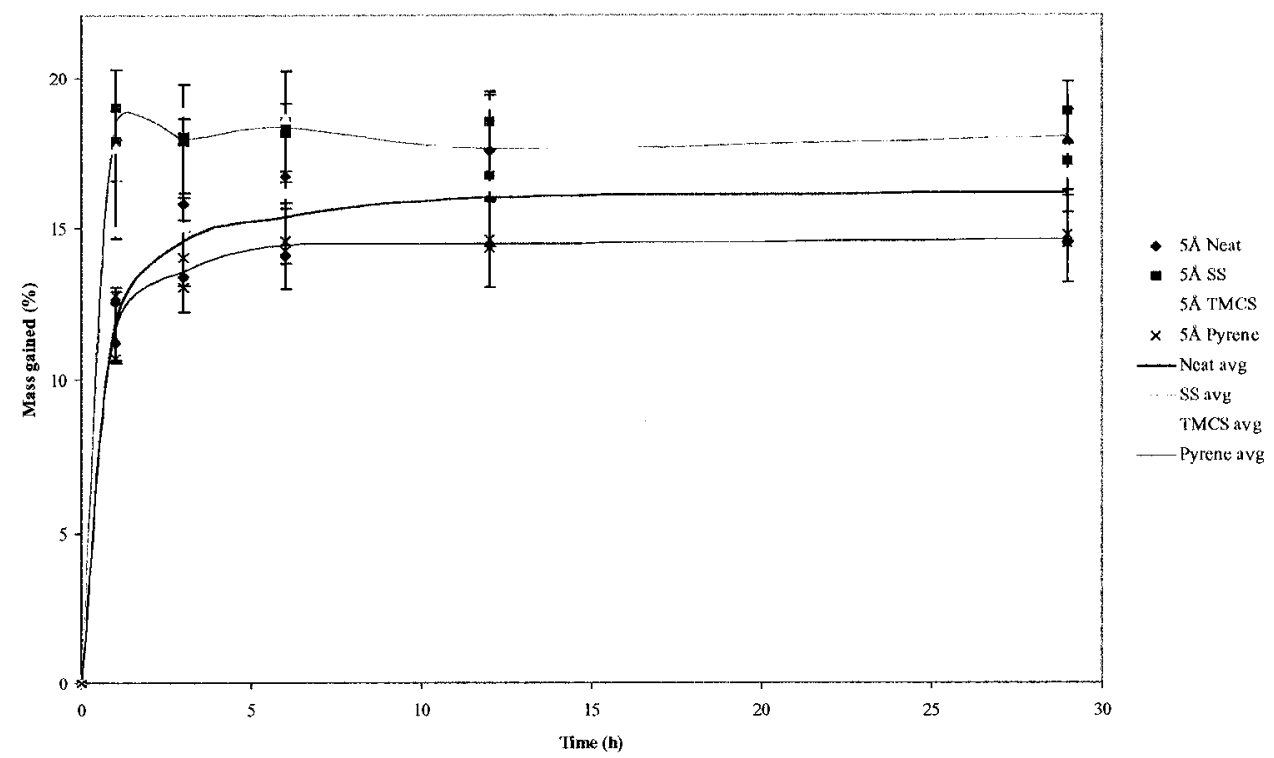

Fig. 4.12 Uptake experiments for LTA $4 \AA$ and LTA $5 \AA \AA$ exposed to water at $97 \%$ humidity.

IV-29 

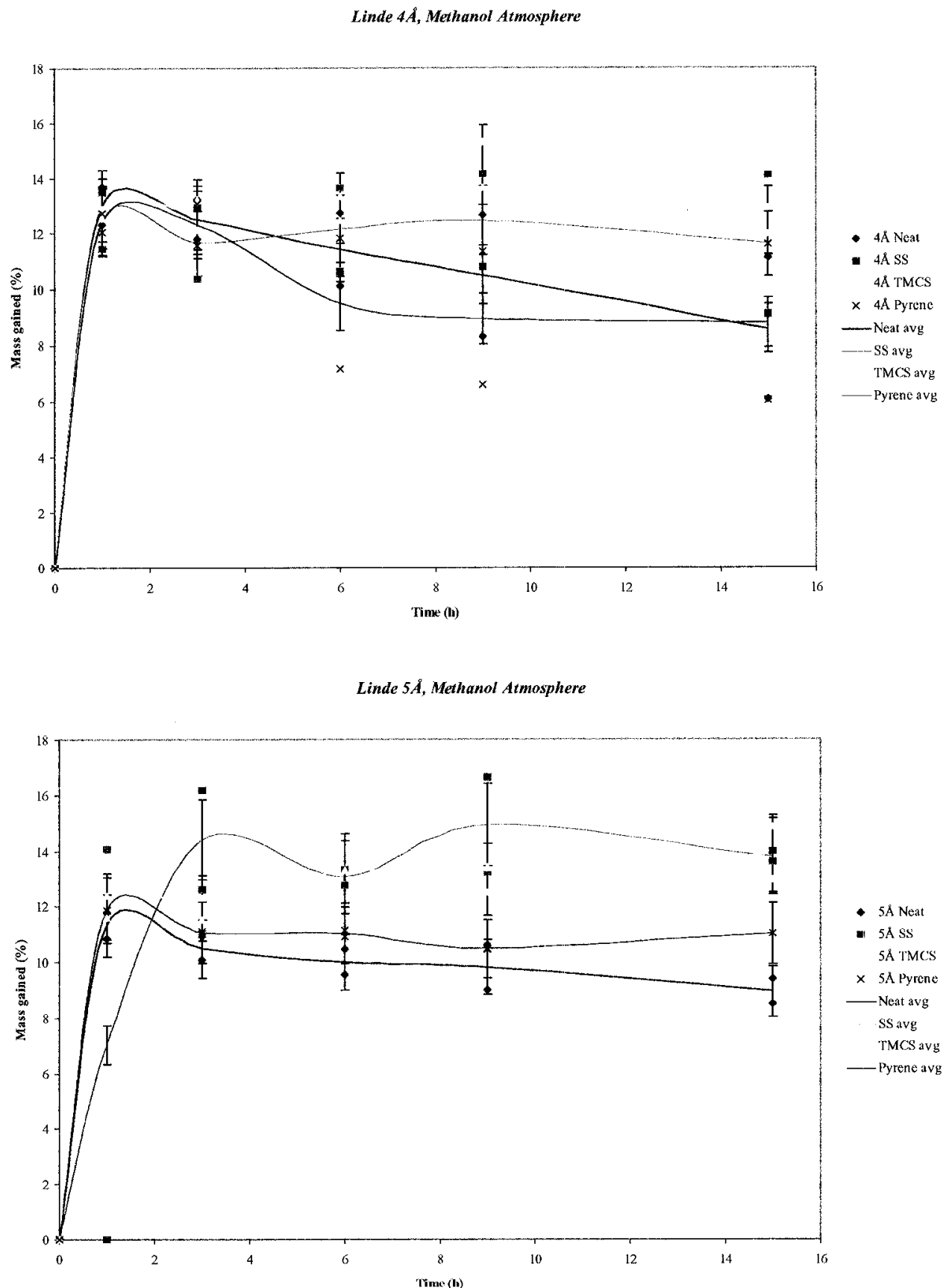

Fig. 4.13 Uptake experiments for LTA $4 \AA$ and LTA $5 \AA$ exposed to methanol.

IV -30 

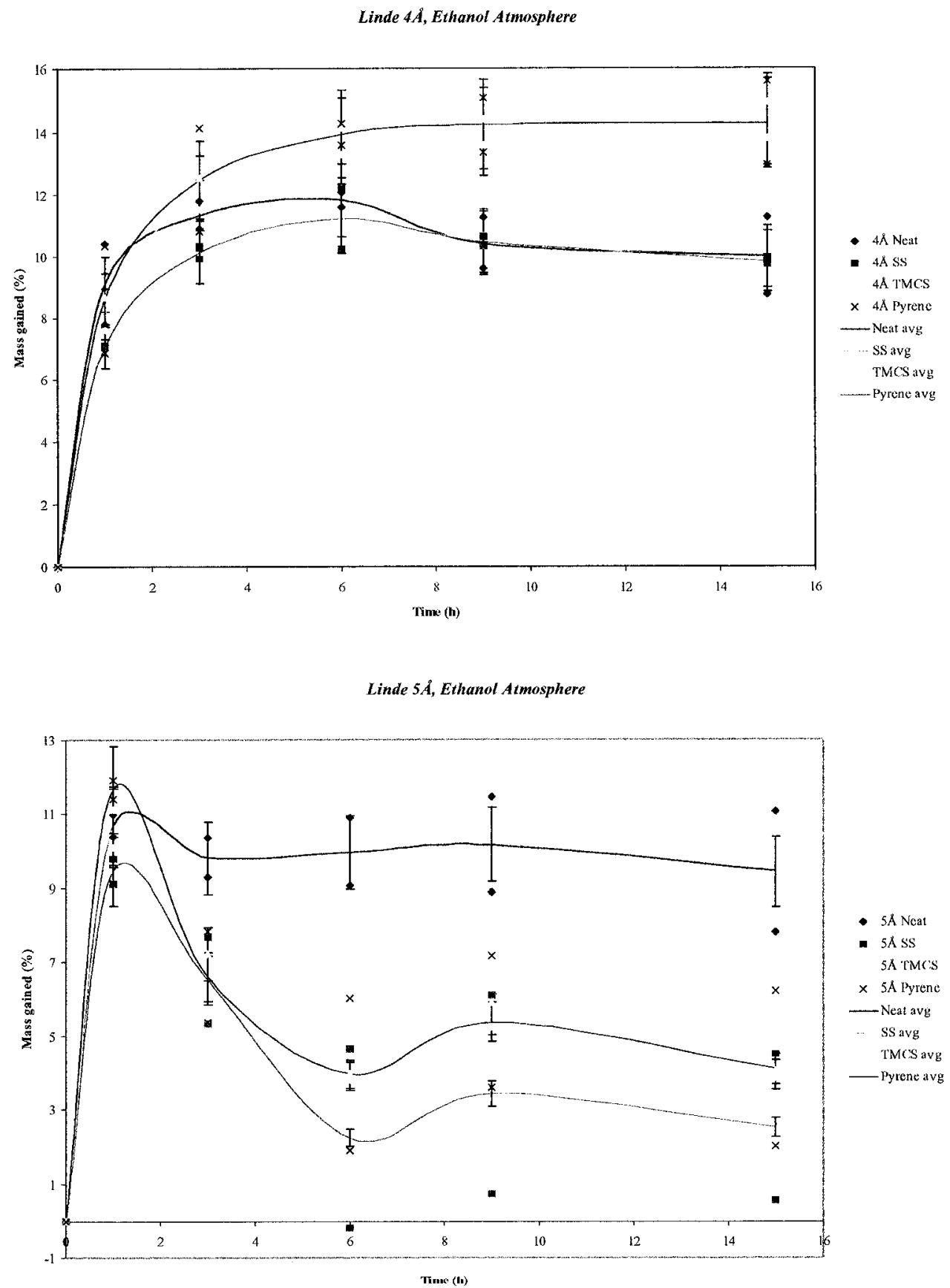

Fig. 4.14 Uptake experiments for LTA $4 \AA$ and LTA $5 \AA$ exposed to ethanol.

IV -31 
respectively. Table 4.7 shows the average ratio of moles of solvent per unit cell of zeolite.

The unit cell for LTA is given as $\left\{\mathrm{Na}_{12}\left[\mathrm{Al}_{12} \mathrm{Si}_{12} \mathrm{O}_{48}\right] \cdot 27 \mathrm{H}_{2} \mathrm{O}\right\}_{8}$, which gives a total of 216 water molecules per unit cell of zeolite. In the case of the water uptake experiments, the amount of water:zeolite ratio was below this number, in the area of 120-160 moles of water to zeolite, which indicates that there is the potential for more uptake if the samples were exposed to the solvent for a greater amount of time. Again, the trend of uptake from water to methanol to ethanol shows a decreasing ratio of solvent to zeolite. This observation can be explained by the simple fact that more water molecules are able to fit in the zeolite structure in contrast to methanol and even less so, ethanol.

The rate of uptake of water is faster than that of methanol and ethanol. Maxima in the water uptake cases are observed within a an hour, whereas the two latter solvents take longer times to reach the same. It could be reasoned that if given sufficient exposure times, all samples would reach the maximum of $20-25 \%$ gained mass. 
Table 4.7 Ratio of moles of solvent per mole of zeolite LTA.

\begin{tabular}{|c|c|c|c|c|}
\hline \multirow{2}{*}{$\begin{array}{l}\text { Zeolite } \\
\text { Sample }\end{array}$} & \multirow{2}{*}{$\begin{array}{l}\text { Coating } \\
\text { material }\end{array}$} & \multicolumn{3}{|c|}{ Solvent Atmosphere } \\
\hline & & $\mathbf{H}_{2} \mathbf{O}$ & МeOH & EtOH \\
\hline & Neat & $125.11 \pm 10.03$ & $36.56 \pm 15.12$ & $29.57 \pm 5.25$ \\
\hline LTA & SS & $157.70 \pm 19.98$ & $49.47 \pm 15.09$ & $29.12 \pm 0.48$ \\
\hline \multirow[t]{3}{*}{$4 \AA$} & TMCS & $135.92 \pm 0.75$ & $53.05 \pm 2.85$ & $42.56 \pm 2.46$ \\
\hline & Pyrene-Cl & $160.20 \pm 0.40$ & $37.52 \pm 16.7$ & $42.25 \pm 5.53$ \\
\hline & Neat & $119.87 \pm 17.53$ & $37.28 \pm 2.53$ & $27.38 \pm 6.69$ \\
\hline LTA & SS & $133.83 \pm 8.61$ & $57.56 \pm 1.09$ & $7.36 \pm 8.10$ \\
\hline \multirow{2}{*}{$5 \AA$} & TMCS & $127.63 \pm 4.18$ & $58.01 \pm 5.65$ & $11.49 \pm 0.37$ \\
\hline & Pyrene-Cl & $108.47 \pm 1.32$ & $45.98 \pm 0.01$ & $11.93 \pm 8.65$ \\
\hline
\end{tabular}




\section{References}

1. Clifton, R.A., Natural and Synthetic Zeolites, Bureau of Mines, 1987, IC 9140.

2. Baerlocher, C., W.M. Meier, and D.H. O1son, Atlas of Zeolite Framework Types. 5th Revised ed. 2001: Elsevier.

3. Hersh, C.K., Molecular Sieves. 1961, London: Reinhold Publishing Corporation.

4. Standards provided by the Micrometrics company of molecular sieve pellets have values of $100-200 \mathrm{~m} 2 / \mathrm{g}$.

5. Engelhardt, G. and D. Michel, High-Resolution Solid-State NMR of Silicates and Zeolites. 1987, Norwich: John Wiley \& Sons Ltd. 
Chapter V

Conclusions

1. Concluding remarks $\ldots \ldots \ldots \ldots \ldots \ldots \ldots \ldots \ldots \ldots \ldots \ldots-2$

2. Future work $\ldots \ldots \ldots \ldots \ldots \ldots \ldots \ldots \ldots \ldots \ldots \ldots$ 
Chapter V Conclusions

\section{Concluding remarks}

In general, the modification of the surfaces of LTA, NS1 and NS2 was successful. Preliminary tests of immersing the coated zeolite material into water, showed that the material floated and formed an aggregated mass on the surface of the water. Zeolite LTA has been known to uptake water, methanol and ethanol into its structure, and it was intended by modifying the zeolite surface to enhance its hydrophobicity. TGA analysis first showed that the modified as well as the neat zeolite samples showed the ability to uptake gaseous guests. Cycling experiments have shown that zeolites are robust to a cycling of temperatures, and show repeated uptake of flow gas without degradation to structure or loss of coating. Conclusions from these experiments are that no appreciable difference is noted between the coated and neat samples with respect to gas uptake. Subsequent uptake experiments with water, methanol and ethanol as solvents also did not show any appreciable difference in uptake in all samples, though it could be stated that within the time frame of the experiment, the samples showed preference in the following relationship: water $>$ methanol $>$ ethanol. The same relationship is also observed for the rate of uptake.

${ }^{13} \mathrm{C}$ SS NMR of the SS-coated LTA $4 \AA$ sample, verified the presence of coating material in that a peak was observed at $0 \mathrm{ppm}$. TGA experiments were done to observe the burning off of the organic coating material by exposing the coated samples to temperatures up to $1000^{\circ} \mathrm{C}$. Loss of mass was observed for the coated materials at temperatures ranging from $250-550^{\circ} \mathrm{C}$. 
There was quite the substantial uptake of helium by both the LTA $4 \AA$ and $5 \AA$ neat samples. Helium, a small atom, has unhindered access to the zeolite pores and channels. This allows for the continuation along that line of thought to perhaps attempt the same uptake experiment, but with hydrogen as the flow gas, to determine whether the uptake is even more substantial. This could prove to be very lucrative for hydrogen storage or fuel cell capabilities. Heating of the zeolite to release the gas does not have to exceed that of the boiling point of water to extract all gas from the sample, if this method of gas storage were to become commercialized.

The natural samples, NS1 and NS2 showed ease in instrumental analysis, but proved elusive when identification was attempted. ${ }^{29} \mathrm{Si} \mathrm{NMR}$ showed that 5 different Si species were present in both cases. In comparison of the spectra to those in the literature, it was determined that impurities were present in the sample. Conversely, ${ }^{27} \mathrm{Al}$ NMR spectra for these samples were simple to analyze: all aluminum was tetrahedrally coordinated. PXRD patterns also showed evidence of impurities in the zeolite samples, and patterns obtained for this thesis could not be matched to any known zeolite powder pattern available in the JCPDS database.

The development of a very efficient and straightforward method to prepare a pyrene1-(4-butyl-dimethylchlorosilyl) ether compound has been realized via simple protection using TBDMS, reduction with $\mathrm{NaBH}_{4}$, deprotection through the use of TBAF, and finally, the reaction with DCDMS. Purification and characterization is simple, and reaction conditions 
are mild, while experimental yields are good.

\section{2. $\quad$ Future Work}

Future work in this area could entail studying the effect on a series of organosilane coating materials with varying length, or degree of branching of the organic moiety on the coating material. With varying the coating, a study of various sorbent molecules could also be completed, to determine whether there is a relation between coating chain length and size of sorbent molecule. In addition to covalently bonded coating materials, the area of cationexchanged coating materials could be explored. Some ammonium-substituted compounds such as trimethyl- or triethylammonium come to mind.

Kinetic experiments would also prove interesting to observe the bending and flexing behaviour of the coating materials at various temperatures. This could easily be observed with hyperpolarized ${ }^{129}$ Xe NMR spectroscopy. 
Appendix:

Additional Data 


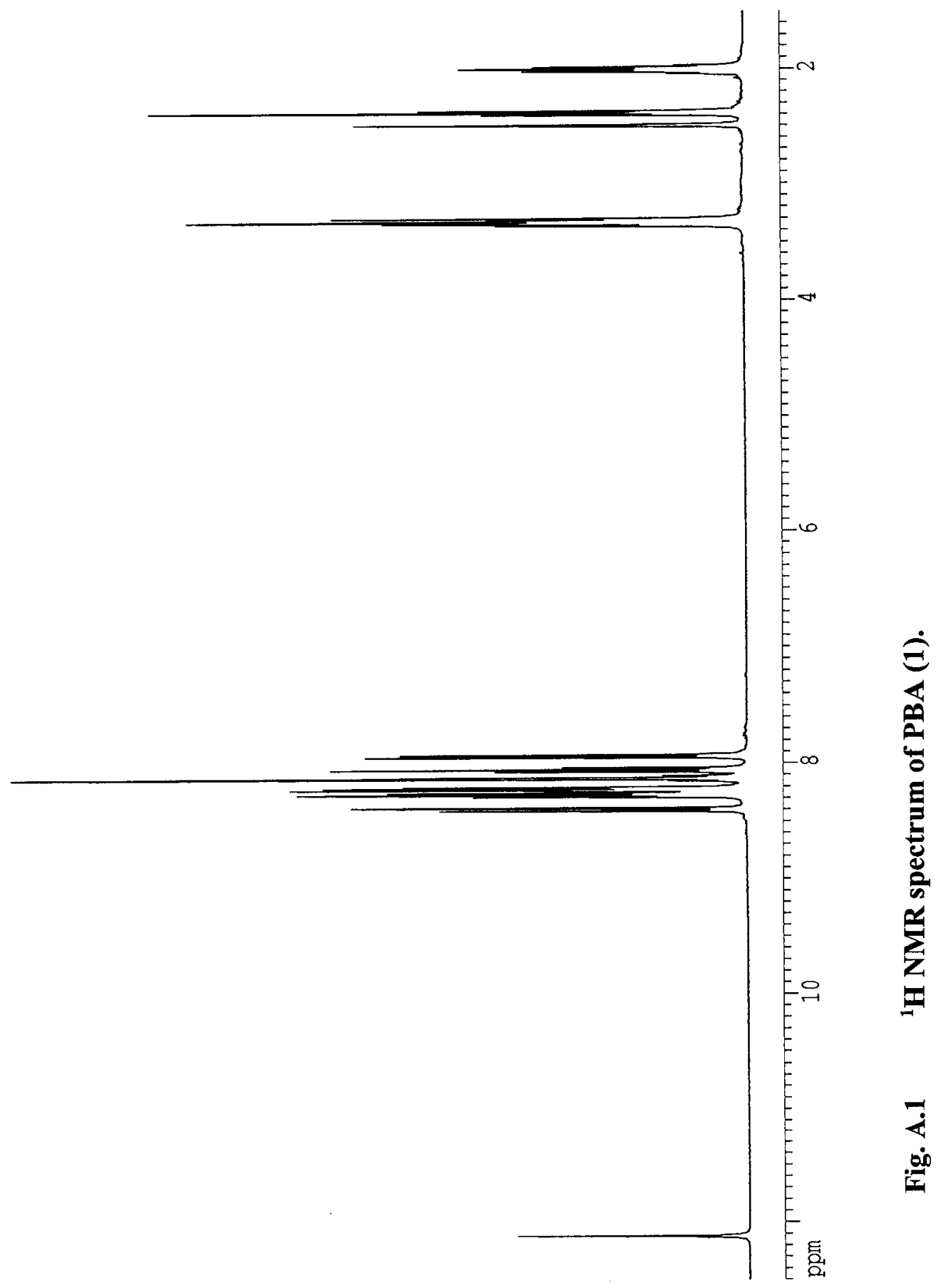

A-2 


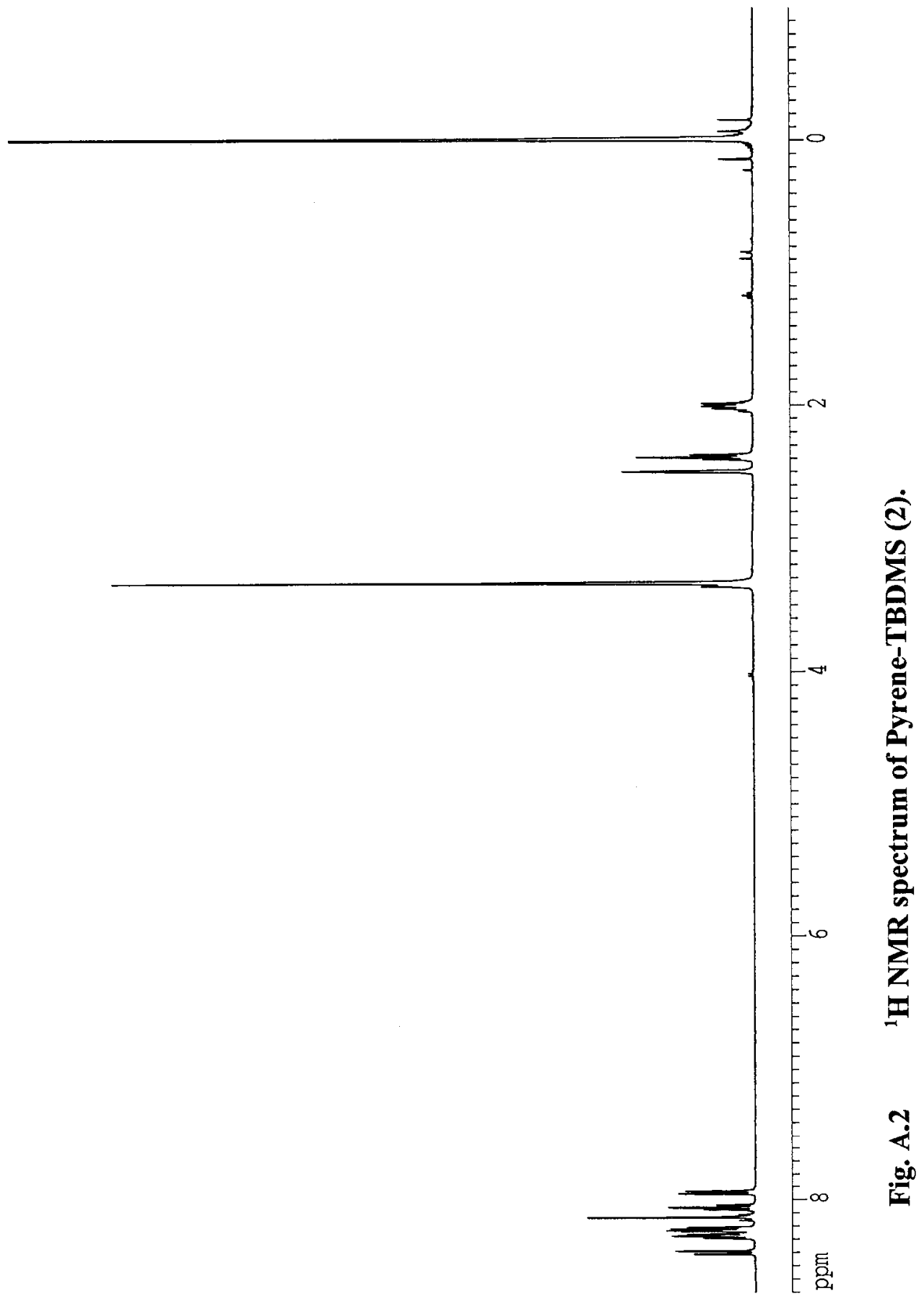

A-3 


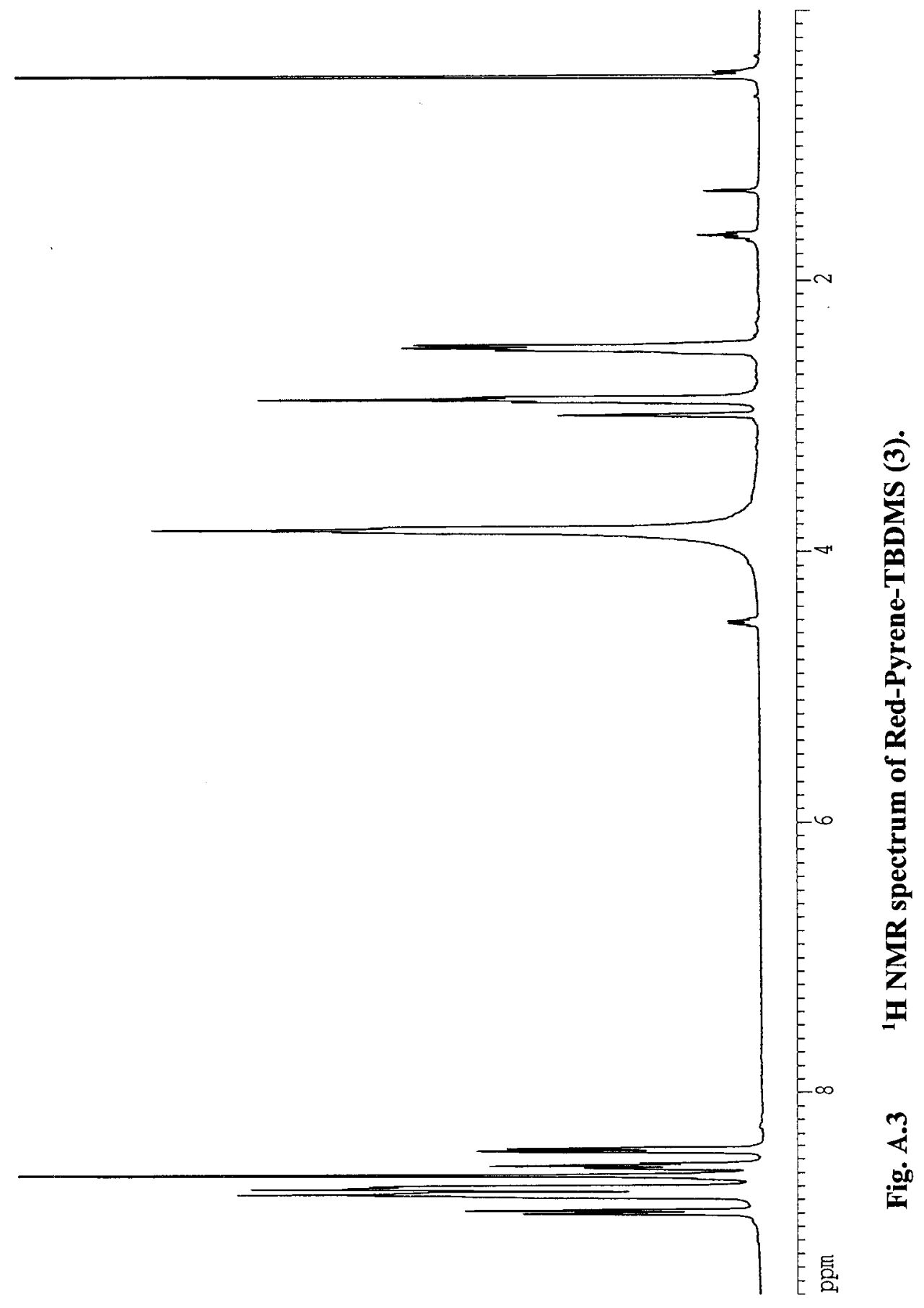

A-4 


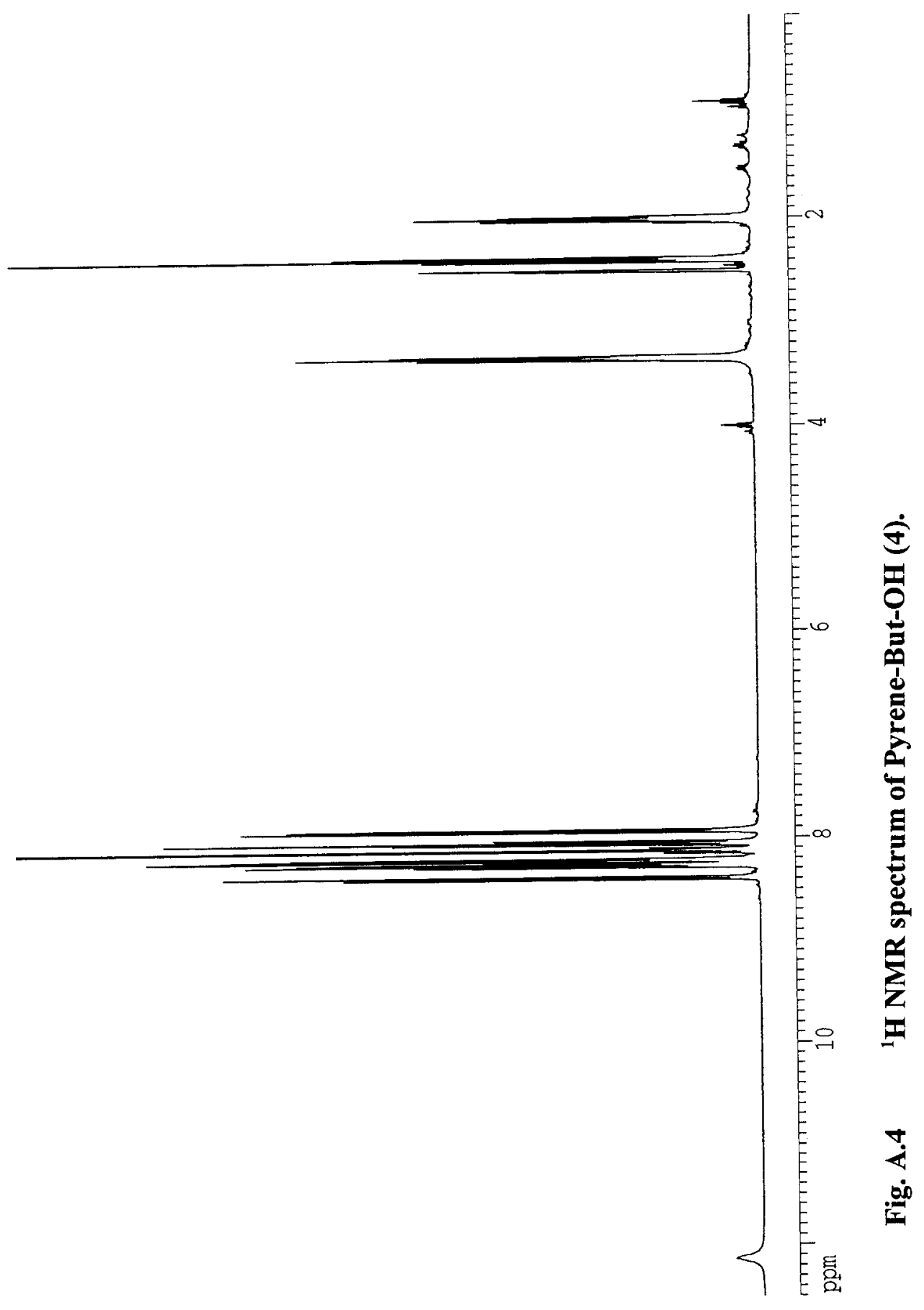

A-5 
Table A.1 PXRD data for MOR.

\begin{tabular}{|c|c|c|c|c|}
\hline 2 Theta & Intensity & $\mathbf{h}$ & $\mathbf{k}$ & 1 \\
\hline 6.446 & 50 & 1 & 1 & 0 \\
\hline 9.711 & 90 & 2 & 0 & 0 \\
\hline 13.384 & 90 & 1 & 1 & 1 \\
\hline 13.869 & 40 & 1 & 3 & 0 \\
\hline 14.509 & 50 & 0 & 2 & 1 \\
\hline 15.290 & 50 & 3 & 1 & 0 \\
\hline 17.618 & 10 & 2 & 2 & 1 \\
\hline 18.201 & 20 & 1 & 3 & 1 \\
\hline 19.580 & 80 & 3 & 3 & 0 \\
\hline 21.446 & 30 & 4 & 2 & 0 \\
\hline 22.206 & 90 & 1 & 5 & 0 \\
\hline 23.143 & 60 & 2 & 4 & 1 \\
\hline 23.643 & 20 & 0 & 0 & 2 \\
\hline 24.571 & 10 & 1 & 1 & 2 \\
\hline 24.992 & 10 & 5 & 1 & 0 \\
\hline 25.576 & 100 & 2 & 0 & 2 \\
\hline 26.267 & 90 & 3 & 5 & 0 \\
\hline 26.914 & 10 & 2 & 2 & 2 \\
\hline 27.681 & 100 & 5 & 1 & 1 \\
\hline 28.775 & 20 & 4 & 4 & 1 \\
\hline 30.314 & 20 & 5 & 3 & 1 \\
\hline 30.850 & 60 & 3 & 3 & 2 \\
\hline 32.618 & 10 & 1 & 5 & 2 \\
\hline 33.152 & 30 & 1 & 7 & 1 \\
\hline 33.941 & 10 & 3 & 7 & 0 \\
\hline 35.022 & 40 & 4 & 6 & 1 \\
\hline 35.567 & 50 & 4 & 4 & 2 \\
\hline 36.418 & 20 & 6 & 4 & 1 \\
\hline 36.852 & 20 & 7 & 1 & 1 \\
\hline 38.387 & 20 & 2 & 8 & 1 \\
\hline 39.151 & 10 & 6 & 2 & 2 \\
\hline 39.581 & 10 & 5 & 7 & 0 \\
\hline 40.452 & 20 & 4 & 8 & 0 \\
\hline 41.744 & 20 & 1 & 9 & 1 \\
\hline 42.548 & 10 & 7 & 1 & 2 \\
\hline 44.209 & 40 & 3 & 9 & 1 \\
\hline 44.855 & 40 & 3 & 5 & 3 \\
\hline 45.353 & 40 & 8 & 4 & 1 \\
\hline 46.458 & 40 & 6 & 8 & 0 \\
\hline 46.890 & 10 & 1 & 9 & 2 \\
\hline
\end{tabular}




\begin{tabular}{lcccc}
47.383 & 20 & 4 & 8 & 2 \\
48.293 & 40 & 0 & 0 & 4 \\
48.789 & 10 & 4 & 10 & 0 \\
49.211 & 10 & 1 & 11 & 0 \\
50.284 & 30 & 4 & 10 & 1 \\
50.824 & 30 & 1 & 11 & 1 \\
51.751 & 10 & 2 & 10 & 2 \\
52.616 & 10 & 10 & 2 & 1 \\
53.210 & 20 & 9 & 3 & 2 \\
53.955 & 10 & 8 & 8 & 0 \\
54.370 & 20 & 5 & 7 & 3 \\
55.114 & 20 & 4 & 8 & 3 \\
55.768 & 10 & 3 & 5 & 4 \\
56.705 & 20 & 9 & 7 & 1 \\
57.675 & 30 & 4 & 12 & 0 \\
59.767 & 20 & 6 & 10 & 4 \\
60.588 & 30 & 6 & 4 & 2 \\
61.434 & 10 & 11 & 1 & 5 \\
62.165 & 5 & 0 & 2 & 1 \\
62.821 & 10 & 9 & 9 & 4 \\
64.426 & 30 & 1 & 9 & 1 \\
65.390 & 20 & 12 & 4 & \\
\hline
\end{tabular}


Table A.2 PXRD data for STI.

\begin{tabular}{|c|c|c|c|c|}
\hline 2 Theta & Intensity & h & $\mathbf{k}$ & 1 \\
\hline 9.691 & 999 & 0 & 2 & 0 \\
\hline 9.911 & 326 & 0 & 0 & 2 \\
\hline 12.993 & 4 & 2 & 0 & 0 \\
\hline 13.878 & 7 & 0 & 2 & 2 \\
\hline 16.234 & 51 & 2 & 2 & 0 \\
\hline 16.367 & 123 & 2 & 0 & 2 \\
\hline 16.712 & 63 & 1 & 2 & 1 \\
\hline 16.970 & 10 & 1 & & 3 \\
\hline 19.055 & 596 & 2 & 2 & 2 \\
\hline 19.452 & 15 & 0 & 4 & 0 \\
\hline 19.896 & 22 & 0 & 0 & 4 \\
\hline 20.756 & 273 & 3 & 1 & 1 \\
\hline 21.888 & 922 & 0 & 4 & 2 \\
\hline 21.888 & & 1 & 3 & 3 \\
\hline 22.174 & 96 & 0 & 2 & 4 \\
\hline 23.463 & 11 & 2 & 4 & 0 \\
\hline 23.836 & 130 & 2 & 0 & 4 \\
\hline 24.966 & 2 & 3 & 3 & 1 \\
\hline 25.143 & 4 & 3 & 1 & 3 \\
\hline 25.526 & 52 & 2 & 4 & 2 \\
\hline 25.784 & 19 & 1 & 5 & 1 \\
\hline 25.784 & & 2 & 2 & 4 \\
\hline 26.157 & 139 & 4 & 0 & 0 \\
\hline 26.264 & 151 & 1 & 1 & 5 \\
\hline 27.955 & 181 & 4 & 2 & 0 \\
\hline 27.955 & & 0 & 4 & 4 \\
\hline 28.034 & 244 & 4 & 0 & 2 \\
\hline 28.752 & 67 & 3 & 3 & 3 \\
\hline 29.444 & 292 & 1 & 5 & 3 \\
\hline 29.747 & 217 & 4 & 2 & 2 \\
\hline 29.747 & & 1 & 3 & 5 \\
\hline 30.038 & 34 & 0 & 0 & 6 \\
\hline 31.058 & 16 & 0 & 6 & 2 \\
\hline 31.633 & 22 & 0 & 2 & 6 \\
\hline 31.846 & 10 & 3 & 5 & 1 \\
\hline 32.225 & 127 & 2 & 6 & 0 \\
\hline 32.225 & & 3 & 1 & 5 \\
\hline 33.081 & 94 & 4 & 0 & 4 \\
\hline 33.625 & 3 & 5 & 1 & 1 \\
\hline
\end{tabular}




\begin{tabular}{|c|c|c|c|c|}
\hline 34.328 & 56 & 2 & 2 & 6 \\
\hline 34.328 & & 4 & 4 & 2 \\
\hline 34.550 & 15 & 4 & 2 & 4 \\
\hline 34.952 & 105 & 3 & 5 & 3 \\
\hline 35.212 & 32 & 3 & 3 & 5 \\
\hline 35.407 & 12 & 1 & 7 & 1 \\
\hline 35.720 & 19 & 0 & 6 & 4 \\
\hline 35.792 & 14 & 1 & 5 & 5 \\
\hline 36.039 & 277 & 0 & 4 & 6 \\
\hline 36.174 & 21 & 1 & 1 & 7 \\
\hline 36.598 & 59 & 5 & 1 & 3 \\
\hline 38.161 & 25 & 2 & 6 & 4 \\
\hline 38.260 & 35 & 1 & 7 & 3 \\
\hline 38.463 & 6 & 2 & 4 & 6 \\
\hline 38.665 & 13 & 4 & 4 & 4 \\
\hline 38.859 & 10 & 1 & 3 & 7 \\
\hline 39.259 & 1 & 5 & 3 & 9 \\
\hline 39.684 & 24 & 6 & 0 & 0 \\
\hline 39.684 & & 4 & 6 & 0 \\
\hline 40.245 & 11 & 3 & 7 & 1 \\
\hline 40.245 & & 4 & 0 & 6 \\
\hline 40.426 & 15 & 0 & 0 & 8 \\
\hline 40.541 & 22 & 3 & 5 & 5 \\
\hline 40.885 & 18 & 5 & 1 & 7 \\
\hline 40.948 & 15 & 6 & 2 & 0 \\
\hline 40.948 & & 6 & 0 & 2 \\
\hline 41.495 & 2 & 4 & 2 & 6 \\
\hline 41.671 & 10 & 5 & 5 & 1 \\
\hline 41.671 & & 0 & 2 & 8 \\
\hline 42.000 & 7 & 5 & 1 & 5 \\
\hline 42.236 & 2 & 6 & 2 & 2 \\
\hline 42.501 & 20 & 0 & 6 & 6 \\
\hline 42.639 & 15 & 2 & 0 & 8 \\
\hline 42.777 & 4 & 3 & 7 & 9 \\
\hline 43.017 & 14 & 2 & 8 & 2 \\
\hline 43.324 & 8 & 3 & 3 & 7 \\
\hline 43.488 & 11 & 1 & 7 & 5 \\
\hline 43.813 & 47 & 1 & 5 & 7 \\
\hline 43.813 & & 2 & 2 & 8 \\
\hline 44.175 & 16 & 5 & 5 & 3 \\
\hline
\end{tabular}




\begin{tabular}{|c|c|c|c|c|}
\hline 44.389 & 68 & 5 & 3 & 5 \\
\hline 44.562 & 42 & 6 & 4 & 0 \\
\hline 45.073 & 8 & 4 & 4 & 6 \\
\hline 45.238 & 5 & 0 & 4 & 6 \\
\hline 45.768 & 5 & 6 & 4 & 2 \\
\hline 45.924 & 8 & 6 & 2 & 4 \\
\hline 46.540 & 9 & 1 & 1 & 9 \\
\hline 46.655 & 9 & 2 & 8 & 4 \\
\hline 47.229 & 2 & 7 & 1 & 1 \\
\hline 47.229 & & 2 & 4 & 8 \\
\hline 47.852 & 15 & 1 & 9 & 3 \\
\hline 47.852 & & 3 & 5 & 7 \\
\hline 47.990 & 25 & 4 & 8 & 0 \\
\hline 48.590 & 11 & 5 & 7 & 1 \\
\hline 48.753 & 7 & 1 & 3 & 9 \\
\hline 48.888 & 19 & 5 & 5 & 5 \\
\hline 49.185 & 16 & 4 & 9 & 2 \\
\hline 49.185 & & 5 & 1 & 7 \\
\hline 49.419 & 6 & 7 & 3 & 1 \\
\hline 49.482 & 6 & 3 & 9 & 1 \\
\hline 49.482 & & 7 & 1 & 3 \\
\hline 49.966 & 36 & 0 & 0 & 0 \\
\hline 50.123 & 20 & 6 & 6 & 0 \\
\hline 50.404 & 27 & 0 & 8 & 6 \\
\hline 50.404 & & 3 & 1 & 9 \\
\hline 50.560 & 22 & 6 & 0 & 6 \\
\hline 50.560 & 22 & 4 & 6 & 6 \\
\hline 50.742 & 7 & 0 & 6 & 8 \\
\hline 50.832 & 4 & 5 & 7 & 3 \\
\hline 51.175 & 34 & 0 & 0 & 10 \\
\hline 51.175 & & 6 & 6 & 2 \\
\hline 51.312 & 29 & 5 & 3 & 7 \\
\hline 51.634 & 11 & 7 & 3 & 3 \\
\hline 51.634 & 11 & 3 & 9 & 3 \\
\hline 52.217 & 1 & 0 & 2 & 0 \\
\hline 52.441 & 4 & 4 & 8 & 4 \\
\hline 52.612 & 11 & 3 & 3 & 9 \\
\hline 52.612 & & 2 & 6 & 8 \\
\hline 52.935 & 15 & 2 & 0 & 2 \\
\hline 52.935 & & 1 & 5 & 9 \\
\hline
\end{tabular}




\begin{tabular}{|c|c|c|c|c|}
\hline 53.034 & 8 & 2 & 0 & 10 \\
\hline 53.876 & 28 & 8 & 0 & 0 \\
\hline 53.876 & & 7 & 1 & 5 \\
\hline 54.051 & 16 & 2 & 2 & 10 \\
\hline 54.212 & 15 & 3 & 7 & 7 \\
\hline 54.824 & 18 & 8 & 2 & 0 \\
\hline 54.824 & & 8 & 0 & 2 \\
\hline 55.117 & 32 & 5 & 7 & 5 \\
\hline 55.260 & 20 & 0 & 4 & 10 \\
\hline 55.390 & 6 & 5 & 5 & 7 \\
\hline 55.864 & 21 & 8 & 2 & 2 \\
\hline 55.864 & & 7 & 3 & 5 \\
\hline 56.069 & 14 & 1 & 1 & 1 \\
\hline 56.563 & 5 & 3 & 5 & 9 \\
\hline 57.026 & 6 & 2 & 4 & 10 \\
\hline 57.260 & 11 & 6 & 8 & 0 \\
\hline 57.260 & & 4 & 0 & 0 \\
\hline 57.410 & 7 & 1 & 1 & 11 \\
\hline 57.659 & 8 & 4 & 8 & 6 \\
\hline 57.659 & & 5 & 1 & 9 \\
\hline 57.797 & 25 & 8 & 4 & 0 \\
\hline 57.797 & & 0 & 8 & 8 \\
\hline 57.968 & 64 & 8 & 0 & 4 \\
\hline 57.968 & 64 & 4 & 6 & 8 \\
\hline 58.106 & 35 & 1 & 1 & 3 \\
\hline 58.243 & 17 & 6 & 8 & 2 \\
\hline 58.243 & & 4 & 0 & 2 \\
\hline 58.364 & 9 & 4 & 0 & 0 \\
\hline 58.545 & 5 & 1 & 9 & 7 \\
\hline 58.899 & 21 & 1 & 7 & 9 \\
\hline 58.899 & 21 & 6 & 2 & 8 \\
\hline 59.319 & 30 & 4 & 2 & 0 \\
\hline 59.319 & & 1 & 3 & 1 \\
\hline 59.515 & 31 & 2 & 8 & 8 \\
\hline 59.515 & 31 & 3 & 11 & 1 \\
\hline 59.741 & 11 & 7 & 5 & 5 \\
\hline 60.000 & 2 & 7 & 1 & 7 \\
\hline 60.099 & 2 & 0 & 6 & 10 \\
\hline 60.629 & 2 & 3 & 1 & 11 \\
\hline 61.097 & 8 & 2 & 10 & 6 \\
\hline
\end{tabular}




\begin{tabular}{|c|c|c|c|c|}
\hline 61.097 & & 5 & 7 & 7 \\
\hline 61.240 & 6 & 6 & 8 & 4 \\
\hline 61.240 & & 4 & 10 & 4 \\
\hline 61.446 & 5 & 7 & 7 & 3 \\
\hline 61.446 & & 3 & 11 & 3 \\
\hline 61.723 & 5 & 6 & 4 & 8 \\
\hline 61.723 & 5 & 8 & 4 & 4 \\
\hline 61.925 & 3 & 0 & 12 & 2 \\
\hline 61.925 & 3 & 3 & 9 & 7 \\
\hline 62.053 & 5 & 1 & 11 & 5 \\
\hline 62.264 & 5 & 3 & 7 & 9 \\
\hline 62.489 & 6 & 0 & 0 & 12 \\
\hline 62.489 & & 8 & 6 & 0 \\
\hline 62.685 & 5 & 3 & 3 & 11 \\
\hline 62.758 & 5 & 5 & 9 & 5 \\
\hline 62.867 & 6 & 8 & 0 & 6 \\
\hline 63.063 & 11 & 1 & 5 & 11 \\
\hline 63.345 & 6 & 5 & 5 & 9 \\
\hline 63.345 & & 0 & 2 & 12 \\
\hline 63.448 & 6 & 8 & 6 & 2 \\
\hline 63.615 & 4 & 9 & 1 & 3 \\
\hline 64.075 & 4 & 2 & 0 & 12 \\
\hline 64.5 & 27 & 4 & 8 & 8 \\
\hline 64.749 & 16 & 0 & 12 & 4 \\
\hline 64.981 & 5 & 2 & 2 & 12 \\
\hline 65.427 & 15 & 9 & 3 & 3 \\
\hline 65.52 & 15 & 7 & 5 & 7 \\
\hline 65.618 & 11 & 6 & 10 & 0 \\
\hline 66.141 & 8 & 5 & 11 & 1 \\
\hline 66.141 & & 0 & 10 & 8 \\
\hline 66.309 & 10 & 3 & 5 & 11 \\
\hline 66.309 & 10 & 2 & 12 & 4 \\
\hline 66.553 & 8 & 0 & 8 & 10 \\
\hline 66.553 & 8 & 6 & 10 & 2 \\
\hline 66.64 & 5 & 6 & 0 & 10 \\
\hline 66.64 & & 4 & 6 & 10 \\
\hline 66.818 & 3 & 7 & 9 & 1 \\
\hline 67.235 & 6 & 1 & 13 & 1 \\
\hline 67.427 & 10 & 4 & 12 & 0 \\
\hline 67.427 & 10 & 9 & 1 & 5 \\
\hline
\end{tabular}




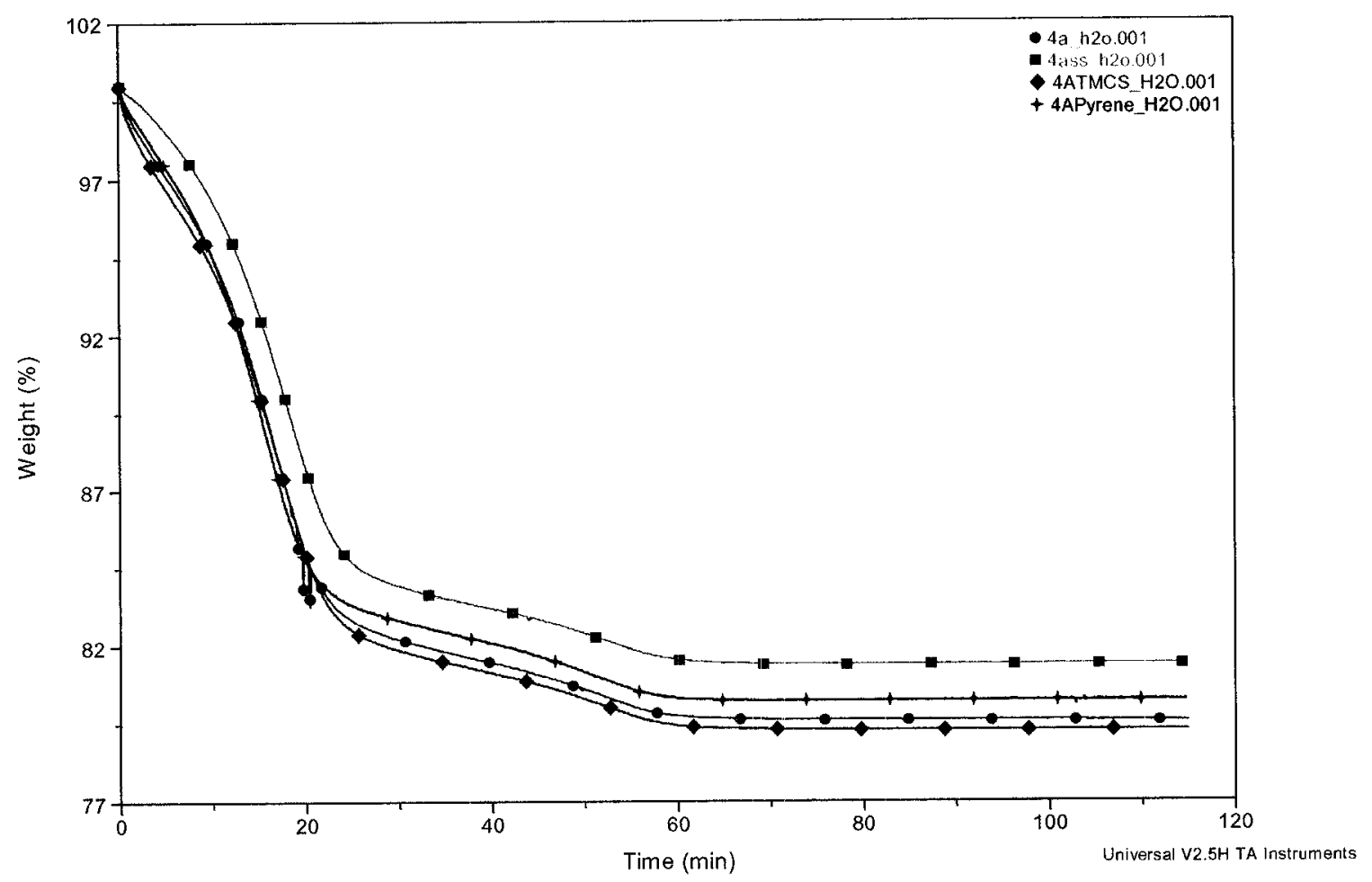

Fig. A.6 Weight loss experiment TGA plot for LTA $4 \AA$ exposed to water vapour. 


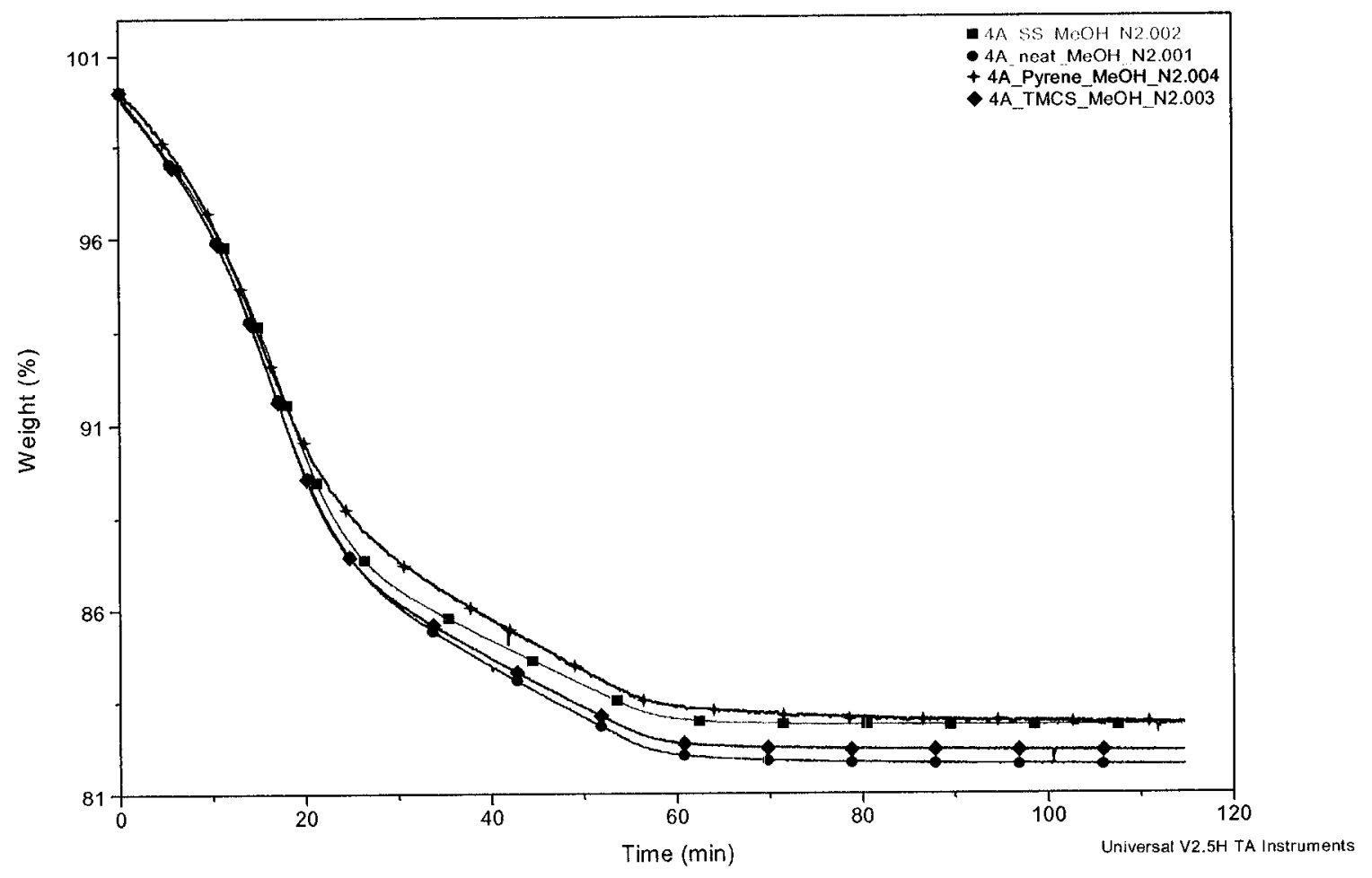

Fig. A.7 Weight loss experiment TGA plot for LTA $4 \AA \AA$ exposed to methanol. 


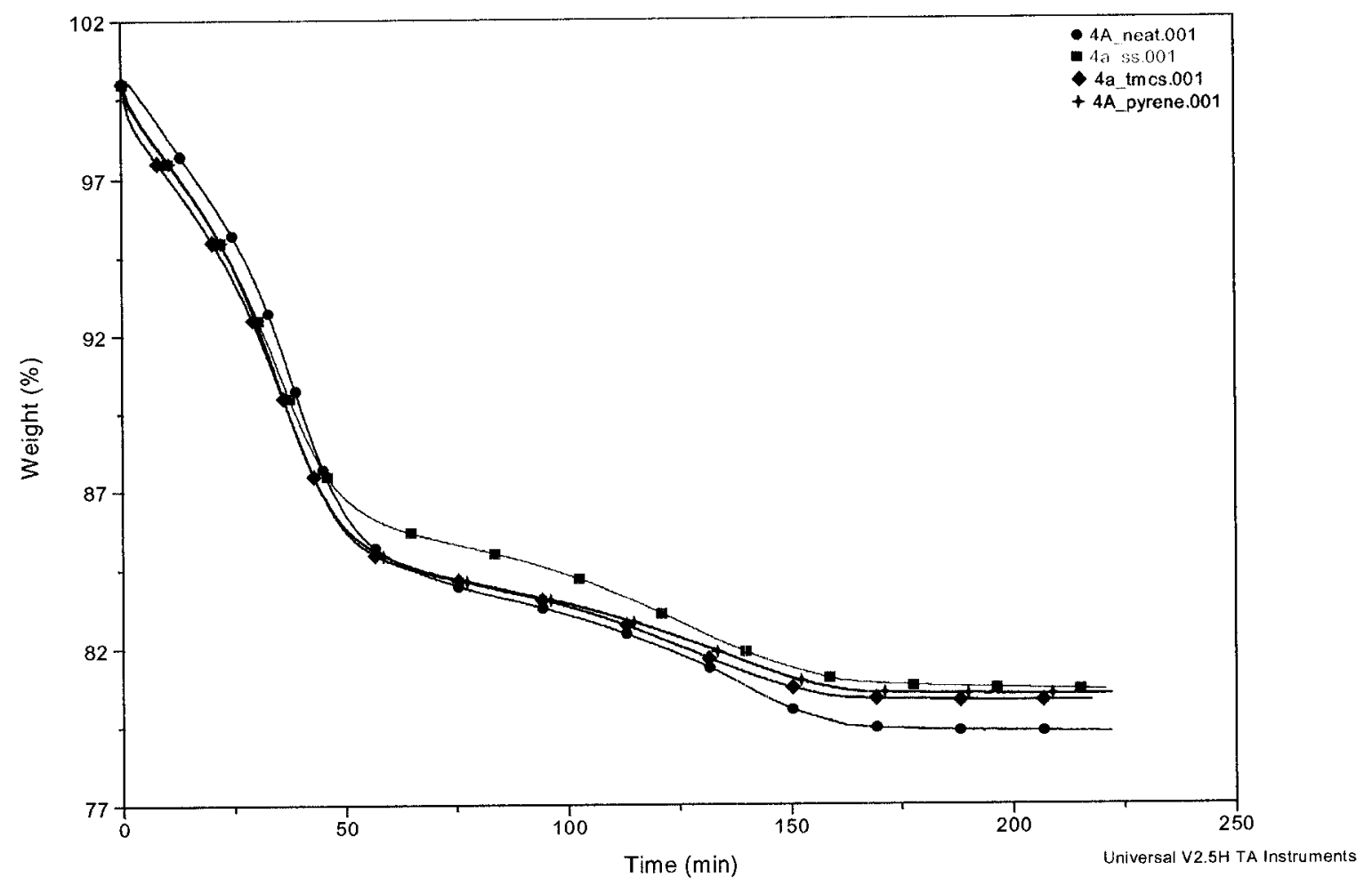

Fig. A.8 Weight loss experiment TGA plot for LTA $4 \AA$ exposed to ethanol. 


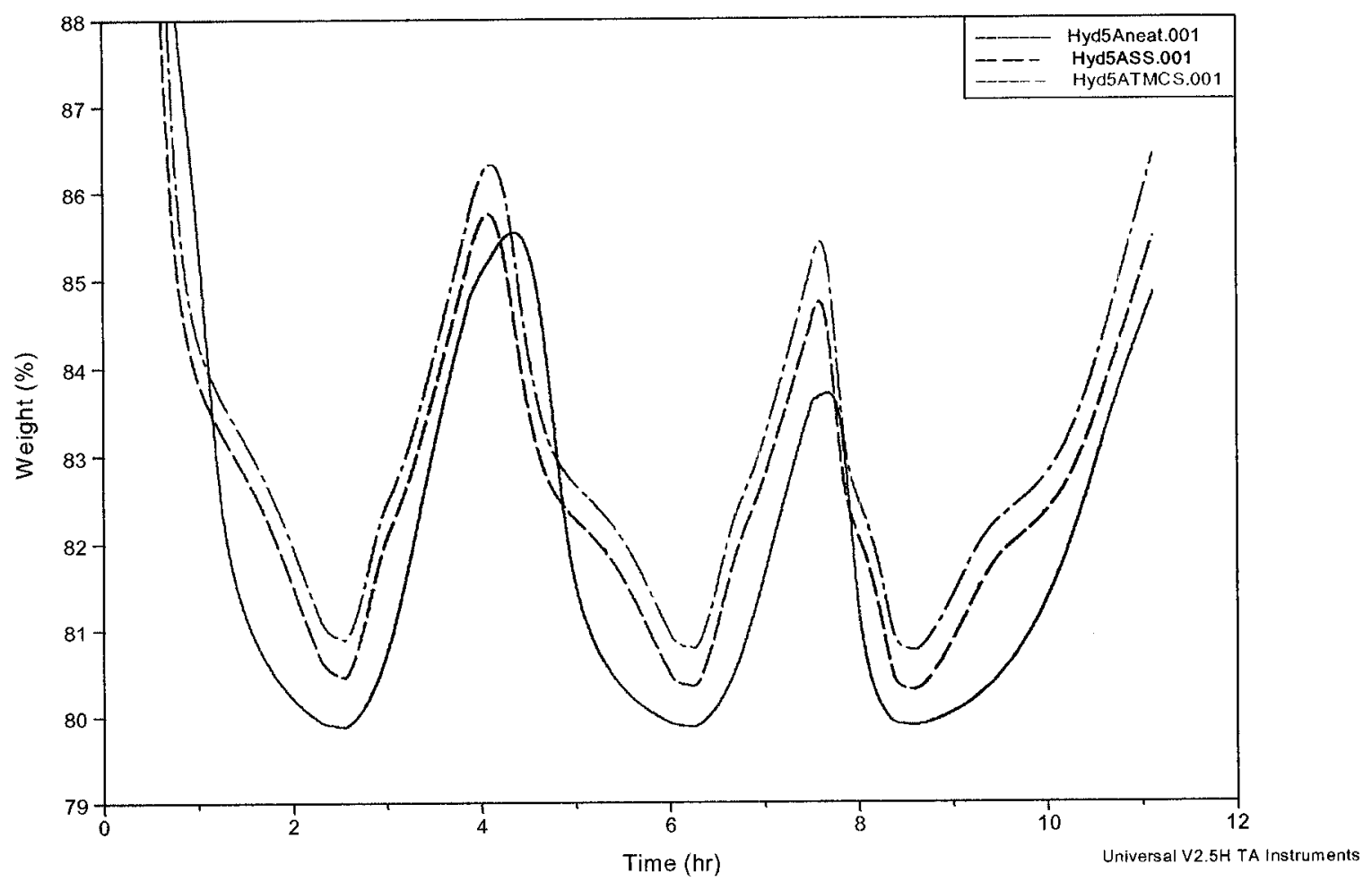

Fig. A.9 Temperature cycle experiment TGA plot for LTA $5 \AA$. 


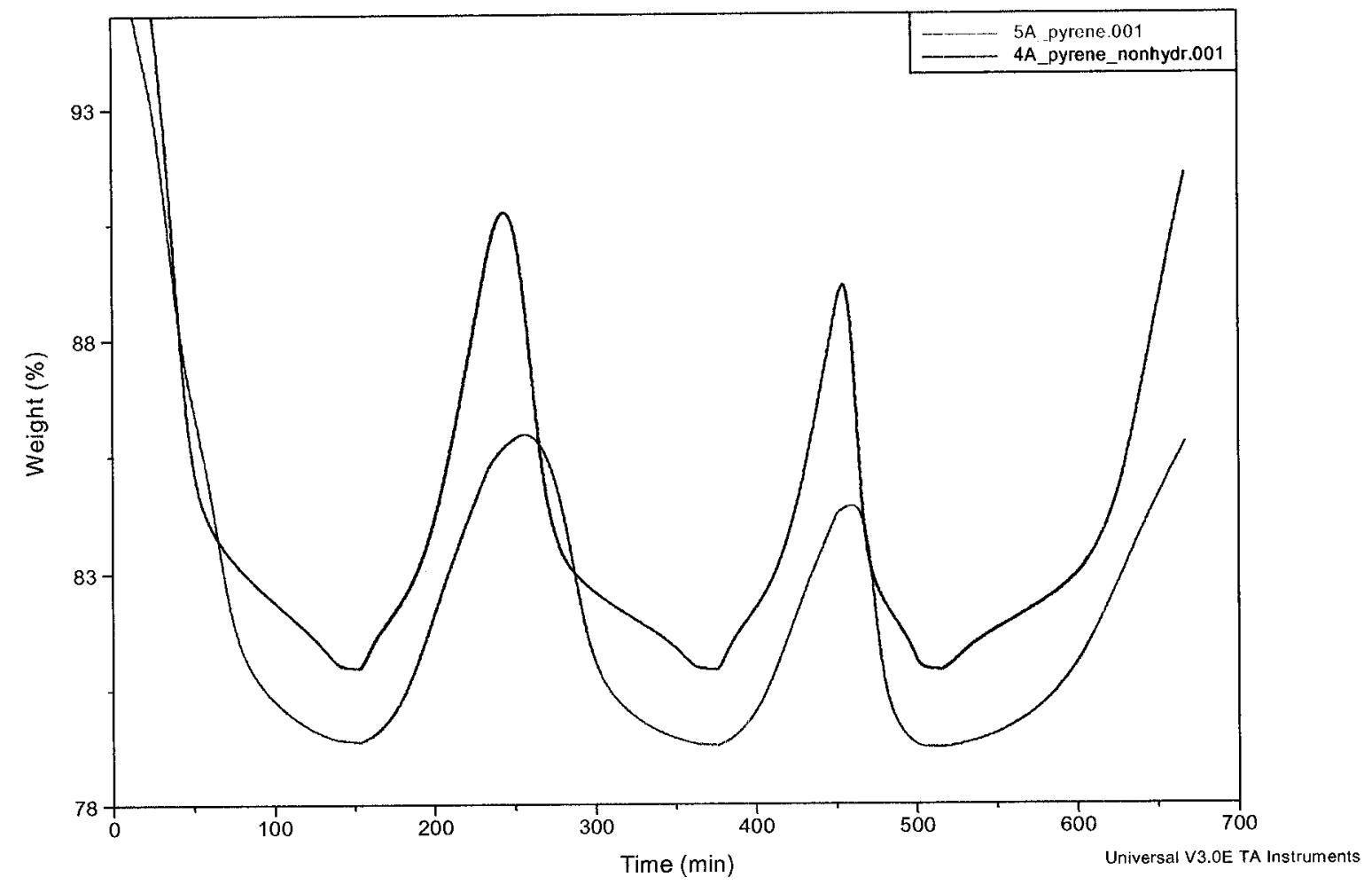

Fig. A.10 Temperature cycle experiment TGA plot for LTA $4 \AA$ and $5 \AA$, coated with Pyrene-Cl. 


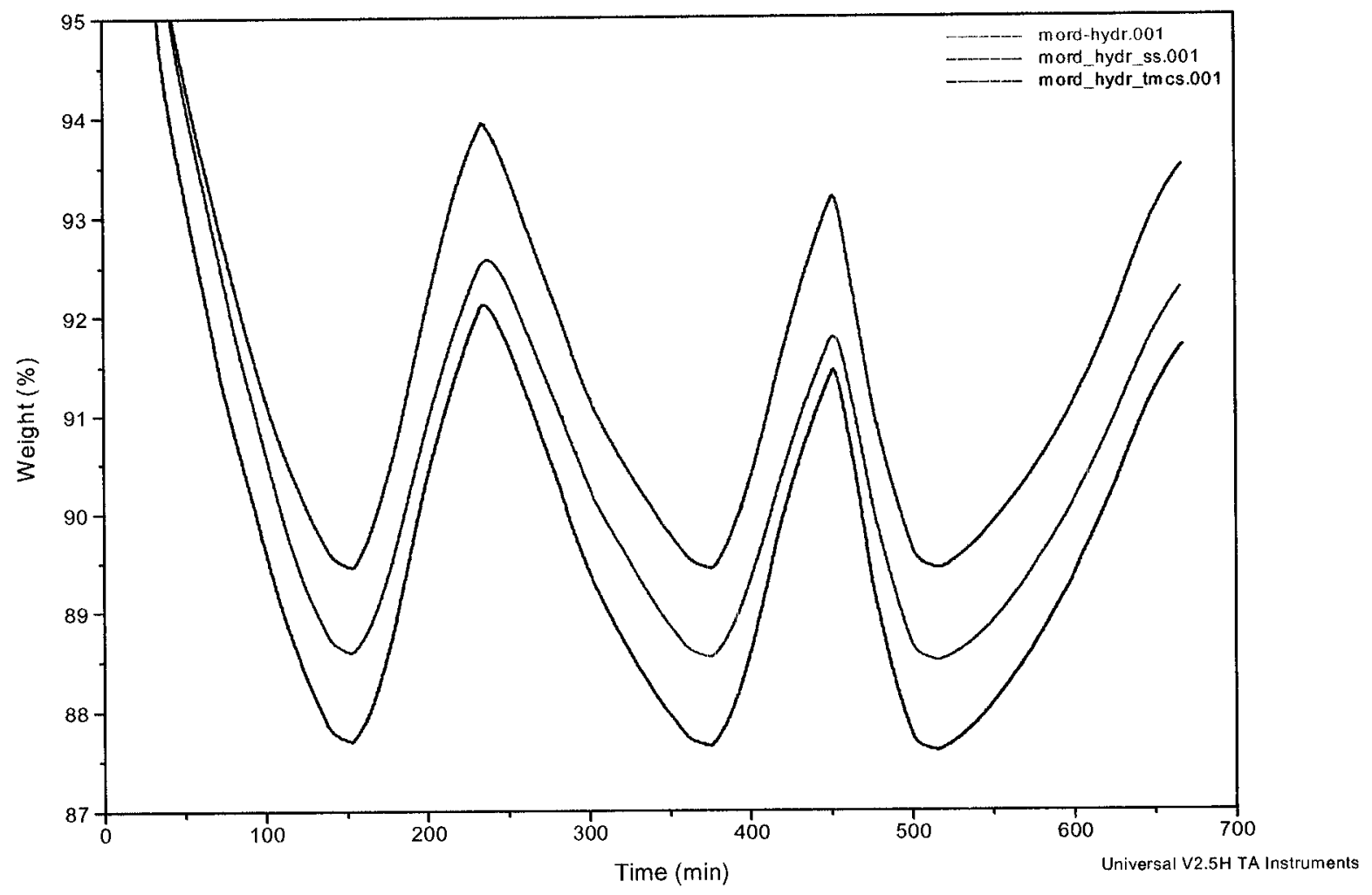

Fig. A.11 Temperature cycle experiment TGA plot for MOR. 


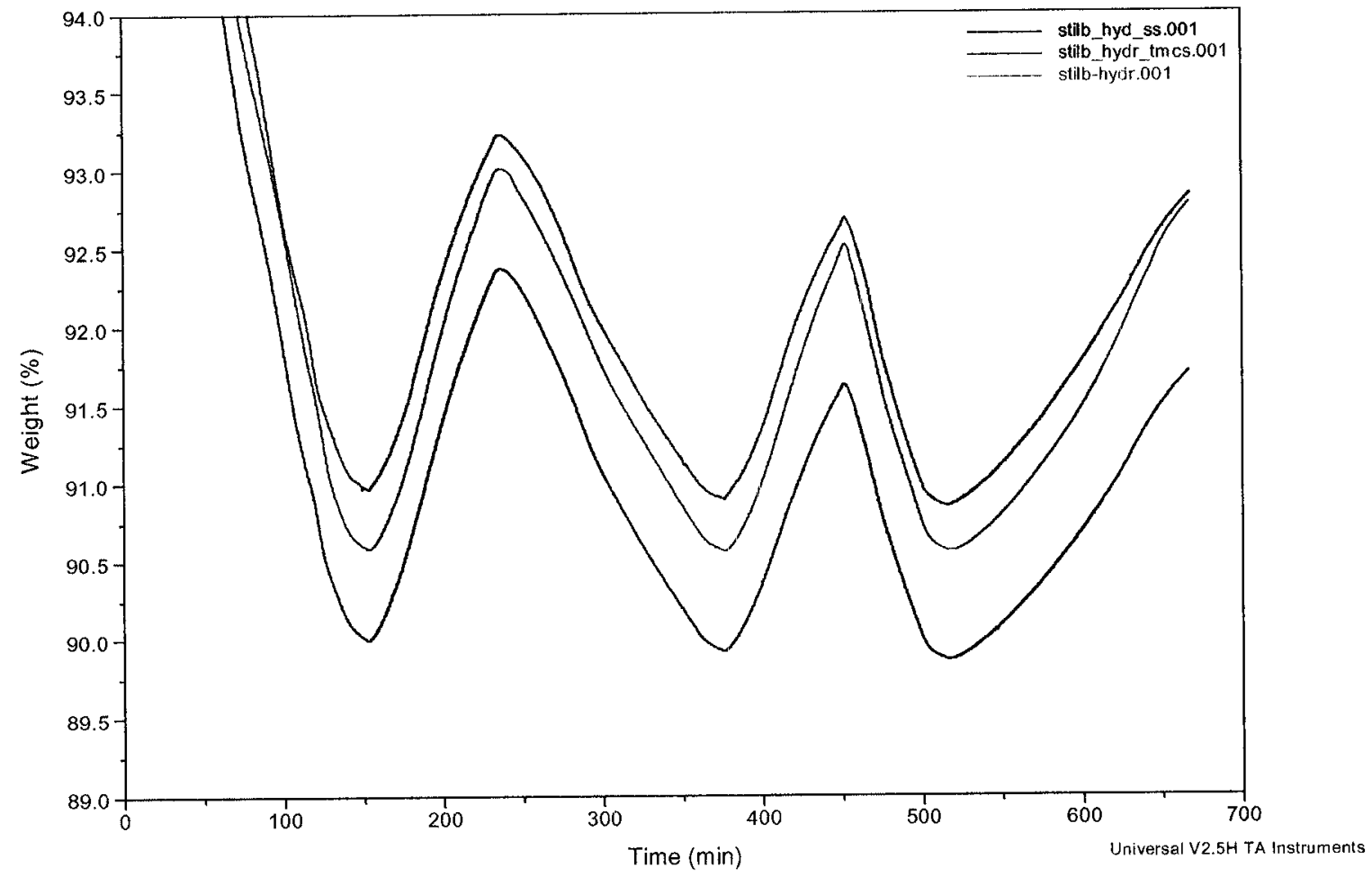

Fig. A.12 Temperature cycle experiment TGA plot for STI. 


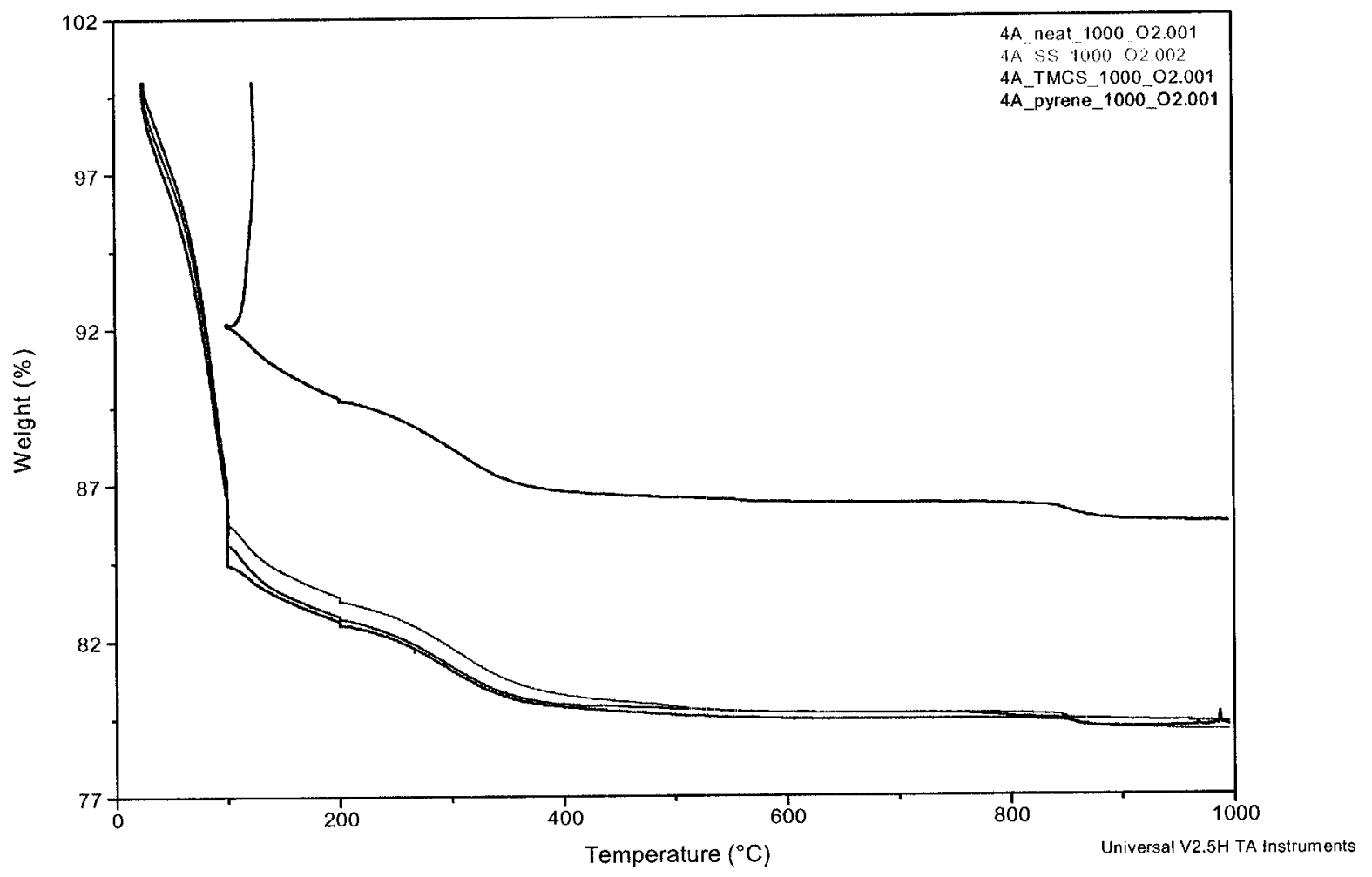

Fig. A.13 Weight loss experiment TGA plot for $4 \AA$, where the final temperature was $1000^{\circ} \mathrm{C}$, under oxygen flow. 


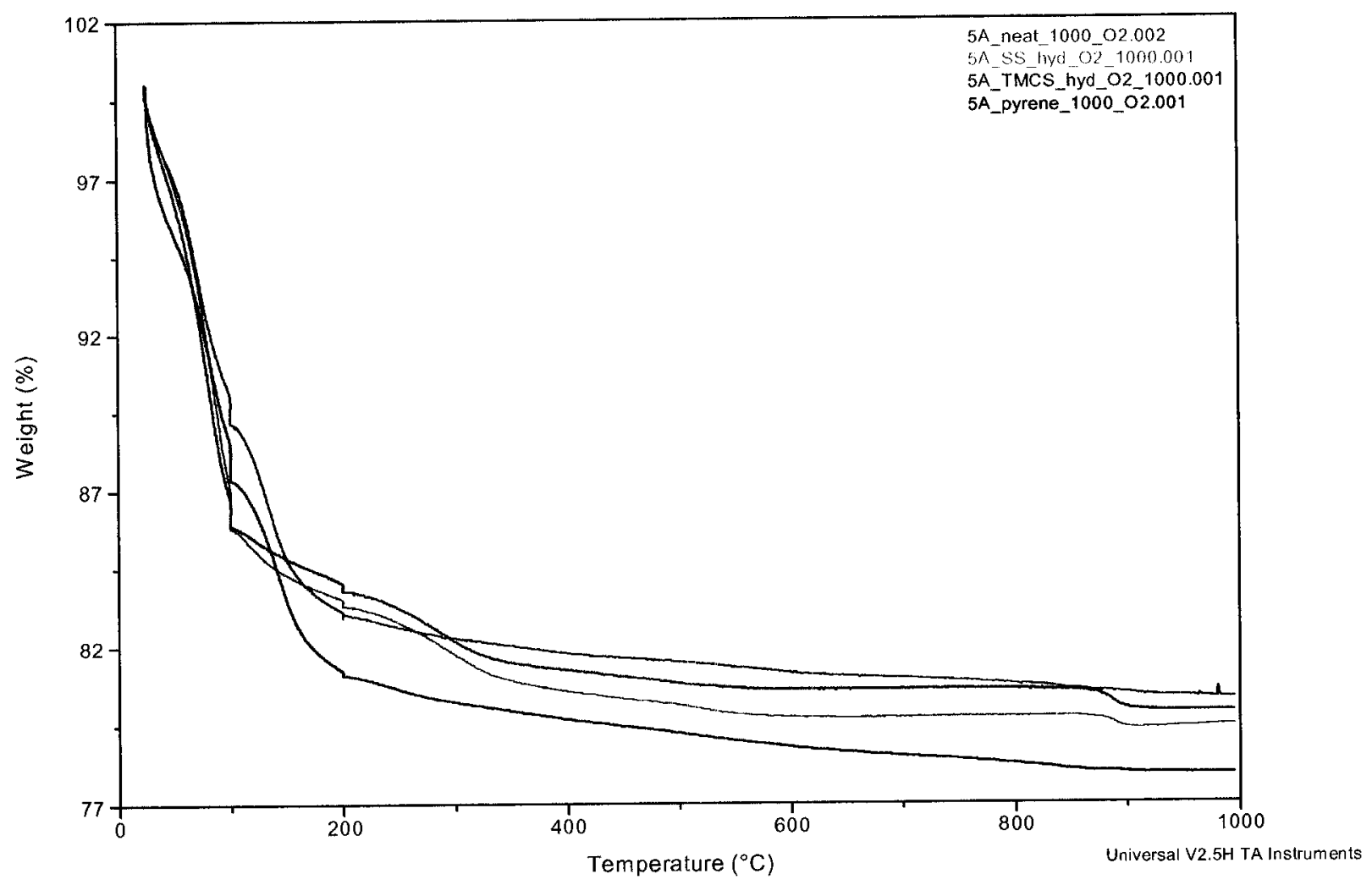

Fig. A.14 Weight loss experiment TGA plot for $5 \AA$, where the final temperature was $1000^{\circ} \mathrm{C}$, under oxygen flow. 


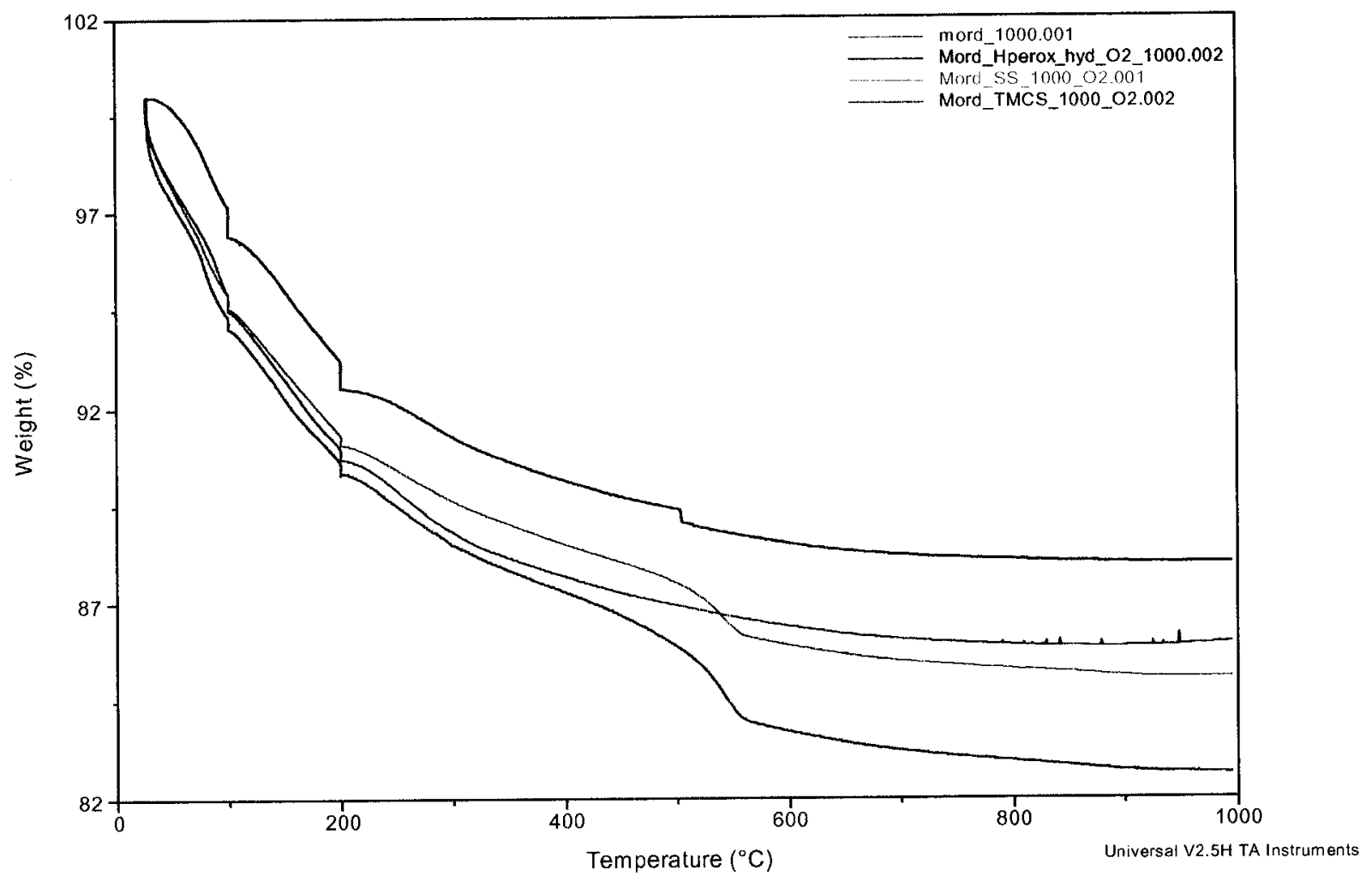

Fig. A.15 Weight loss experiment TGA plot for MOR, where the final temperature was $1000^{\circ} \mathrm{C}$, under oxygen flow. 


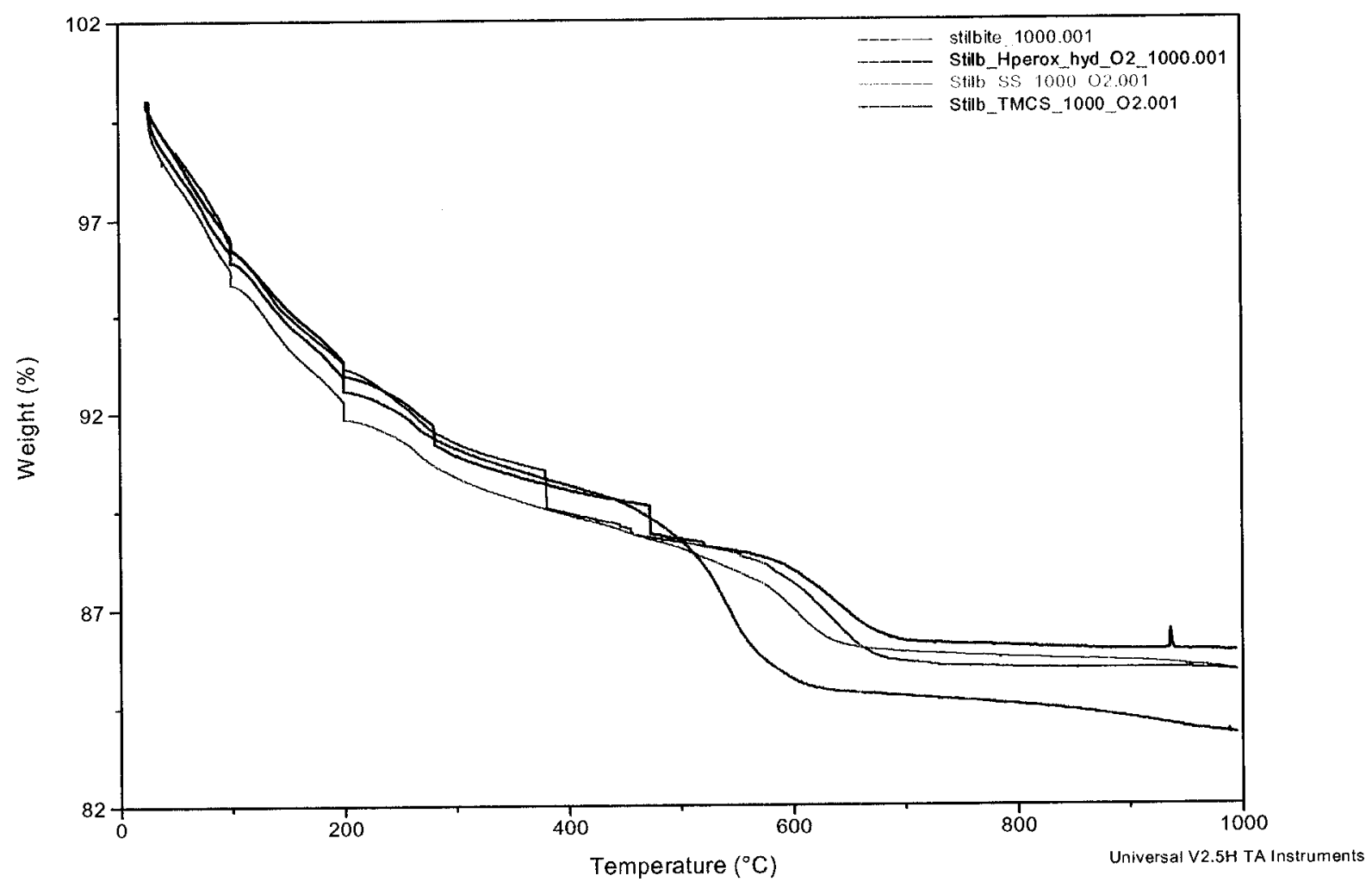

Fig. A.16 Weight loss experiment TGA plot for STI, where the final temperature was $1000^{\circ} \mathrm{C}$, under oxygen flow. 